\title{
DIFFRACTIVE $Z / \gamma^{*} \rightarrow \mu^{+} \mu^{-}$BOSON PRODUCTION IN PROTON - ANTIPROTON COLLISIONS AT $\sqrt{s}=1.96 \mathrm{TeV}$ in the DØ EXPERIMENT
}

\author{
A Dissertation \\ Submitted to the Physics Department \\ of Universidad de los Andes for the degree of \\ Doctor of Physics \\ by \\ Luis Miguel Mendoza Navas, M.S \\ Carlos Avila, Director of Thesis

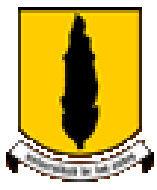 \\ Department of Physics
}

Universidad de los Andes, BOGOTÁ - COLOMBIA

July 2007 
(C) Copyright by

\section{LUIS MIGUEL MENDOZA NAVAS}

2007

All rights reserved 


\section{Contents}

1 Theory 1

1.1 The Standard Model . . . . . . . . . . . . . . . . . . . . . 1

$1.2 Z^{0}$ Bosons at the Tevatron . . . . . . . . . . . . . . 2

2 Experimental Apparatus $\quad 4$

2.1 The Fermilab accelerators . . . . . . . . . . . . . . . . . . 4

2.1.1 The Pre-accelerator . . . . . . . . . . . . . 4

2.1 .2 The Linac . . . . . . . . . . . . . . . . 6

2.1 .3 The Booster . . . . . . . . . . . . . . . 6

2.1 .4 The Main Injector . . . . . . . . . . . . . . . . 6

2.1.5 The Antiproton Source . . . . . . . . . . . . 7

2.1 .6 The Tevatron . . . . . . . . . . . . . . . 7

2.2 Interactions of Energetic Particles with Matter . . . . . . . . 8

2.2.1 Electrons and Photons . . . . . . . . . . . . . 9

2.2 .2 Muons . . . . . . . . . . . . . . . . . . . 9

2.2.3 Hadronic Particles . . . . . . . . . . . . . . . . . . . 9

2.2 .4 Neutrinos . . . . . . . . . . . . . . . . . 10

2.3 The $\mathrm{D} \varnothing$ Detector . . . . . . . . . . . . . . . 10

2.3.1 Coordinate System . . . . . . . . . . . . . . . . 11

2.3.2 Luminosity Monitor . . . . . . . . . . . . . . . . 13

2.3.3 The Central Tracking System . . . . . . . . . . . . . . . 13

The Silicon Microstrip Tracker (SMT) . . . . . . . . . . 14

Central Fiber Tracker (CFT) . . . . . . . . . . . . . . 17

2.3.4 Solenoid Magnet . . . . . . . . . . . . . . . . 17

2.3.5 Momentum Resolution . . . . . . . . . . . . . . . . . . 19

2.3.6 Pre-shower Detectors . . . . . . . . . . . . . . . . . 20

2.3.7 The Calorimeter System . . . . . . . . . . . . . . . . 21

The Inter-Cryostat Detectors . . . . . . . . . . . . . . 24

Calorimeter Resolution . . . . . . . . . . . 25 
2.3 .8 The Muon System . . . . . . . . . . . . . . . . . 26

Toroid Magnet . . . . . . . . . . . . . . 27

Proportional Drift Tubes . . . . . . . . . . . 27

Mini Drift Tubes . . . . . . . . . . . . . . . . 28

Scintillation Counters . . . . . . . . . . . 28

Shielding . . . . . . . . . . . . . . 29

Performance Muon System _. . . . . . . . . . . 30

2.3.9 Forward Proton Detector (FPD) . . . . . . . . . . 30

2.3.10 Trigger System and Data Acquisition System . . . . . . . . 31

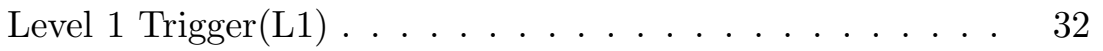

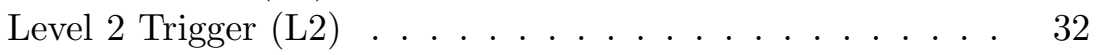

The Level 3 Trigger $(\mathrm{L} 3) \ldots \ldots . \ldots . \ldots 33$

3 Object Identification $\quad 34$

3.1 Charged Tracks . . . . . . . . . . . . . . . . . . 34

3.2 Primary Vertex . . . . . . . . . . . . . . . . . . . 34

3.3 Muons . . . . . . . . . . . . . . . . . . . . . 35

3.4 Electrons . . . . . . . . . . . . . . . . . . . 37

3.5 Jets . . . . . . . . . . . . . . . . . . . . . . 39

3.6 Missing $E_{T}\left(E_{T}\right) \ldots \ldots \ldots \ldots \ldots$

4 Measurement of the Production Cross Section of Diffractive $Z^{0} / \gamma^{*} \rightarrow$ $\mu^{+} \mu^{-}$with Gap 41

4.1 Data Sample . . . . . . . . . . . . . . . . . . . 41

4.2 Selection Criteria . . . . . . . . . . . . . . . . 41

4.3 Trigger Selection . . . . . . . . . . . . . . . . . . . . . . . 47

4.4 Z Diffractive Event Selection . . . . . . . . . . . . . . . . 48

4.4.1 Fraction of the Proton Momentum Loss . . . . . . . . . . . . 48

4.4 .2 Gap Requirement . . . . . . . . . . . . . . . . . . . 49

4.4.3 Cell Energy Thresholds . . . . . . . . . . . . . . . . . 51

4.4 .4 Choice of event kinematics _.............. 54

4.5 Montecarlo . . . . . . . . . . . . . . . . . 58

4.6 Background Subtraction Method . . . . . . . . . . . . . 64

4.7 Diffractive Cross section for Z boson decaying in two muons . . . . 73

4.7.1 Corrections to obtain the Cross Section . . . . . . . . 73

Number of Events . . . . . . . . . . . . . . . 73

Integrated Luminosity . . . . . . . . . . . . . . . . . . 73

Efficiency of MonteCarlo . . . . . . . . . . . . . . 74

Other Efficiencies and backgrounds . . . . . . . . . . 74

4.7 .2 Systematic Errors . . . . . . . . . . . . . . . 75 
Cell Energy Thresholds . . . . . . . . . . . . . . 75

Background . . . . . . . . . . . . . . 75

Gap Size . . . . . . . . . . . . . . . . 76

Integrated Luminosity . . . . . . . . . . . . . . 76

4.7.3 Results for Diffractive Cross Section with Gap . . . . . . . 77

4.8 Fraction of $\mathrm{Z}$ bosons produced diffractively with gap requirement

from $\mathrm{Z}$ inclusive production . . . . . . . . . . . 77

$\begin{array}{ll}\text { A Muon Quality Definitions } & \mathbf{7 9}\end{array}$

$\begin{array}{ll}\text { B Trigger Names } & 81\end{array}$

C Efficiencies and Corrections Factors for Inclusive $\mathrm{Z}$ boson Production $\quad \mathbf{8 2}$

C.1 Efficiency of isolation cuts $\varepsilon_{i s o l} \ldots \ldots \ldots$. . . . . . . . 82

C.2 Efficiency of Opposite Sign Requirement and $b \bar{b}$ Background . . . . 82

C.3 Efficiency and Background for Cosmic Rays . . . . . . . . . . . . 85

C.4 $Z / \gamma^{*} \rightarrow \tau^{+} \tau^{-}$Background . . . . . . . . . . . . . 87

C.5 Background from $\mathrm{W}+$ Jets and Di-boson Events . . . . . . . . 88

D The T42 and Hot Cell Killer Algorithms 89 


\section{List of Tables}

1.1 Schematic representation of the Standard Model: Constituents are doublets of quarks and leptons in three generations. Forces are mediated by gauge bosons . . . . . . 2

1.2 Branching Ratios for $\mathbf{Z}$ boson decays. The invisible decays are presumed to be to neutrinos . . . . . . . . . . 3

2.1 Specifications of the Silicon Vertex Detector . . . . . . . 16

2.2 Pseudorapidity coordinate $|i \eta|$ in the calorimeter . . . . . 23

2.3 Calorimeter Energy Resolution Parameters, measured with electrons for the electromagnetic section ( $\mathbf{C}$ from the $Z \rightarrow e$ e mass resolution, $\mathbf{S}$ from the test beam and $\mathbf{N}$ from $W \rightarrow e \nu$ ) and with pions from the test beam for the hadronic section. . . . . . . 25

4.1 Integrated Luminosity by trigger version . . . . . . . . . . 45

4.2 Number of events - Z selection criteria _ . . . . . . . . . 47

4.3 Convention of numbers used for cell energy thresholds . . 54

4.4 Subversions by Trigger Versions - $\int \mathcal{L} d t$ in $p b^{-1} \ldots \ldots . . .67$

4.5 Normalization constants for PYTHIA . . . . . . . . . . . 67

$4.6 \xi$ reconstructed and $\xi$ corrected, correction factors applied 70

4.7 Efficiencies and backgrounds taken from the inclusive $Z \rightarrow$ $\mu^{+} \mu^{-}$cross section . . . . . . . . . . . . . 74

4.8 Z Diffractive Cross Section by varying cell energy thresholds in $0.05 \mathrm{GeV} \ldots \ldots \ldots \ldots . \ldots \ldots$

4.9 Z Diffractive Cross Section by varying normalization constants for background in $\pm 1 \sigma \ldots \ldots \ldots \ldots$

4.10 Z Diffractive Cross Section by varying gap size . . . . . . 76

4.11 Systematic Errors for fraction of Diffractive $\mathbf{Z}$ boson from $\mathrm{Z}$ inclusive production ............... 78

B.1 Description of trigger components used for trigger names 81 


\section{List of Figures}

$1.1 \mathrm{Z}$ production and decay at the Tevatron . . . . . . . . 3

2.1 The accelerator chain at Fermilab . . . . . . . . . . 5

2.2 Schematic view of magnetron operation for the hydrogen ion source . . . . . . . . . . . . . . . 5

2.3 Schematic drawing of Linac RF cavity . . . . . . . . . . 6

2.4 Simplified drawing of anti-proton production with nickel target and lithium lens. . . . . . . . . . . . . . 7

2.5 The integrated luminosity per week and total integrated luminosity for Run II from May 2001 until May 2007 . . . 8

2.6 Energy loss through ionization of muons in various energy regimes . . . . . . . . . . . . . . . . 10

2.7 Side view of the $\mathrm{D} \varnothing$ detector . . . . . . . . . . . . 11

2.8 Luminosity Monitor layout. The $r-\phi$ view is shown on the left, the $r-z$ view of the two arrays is shown on the right. 13

2.9 The sketch on the left shows the diferentiation between inelastic collisions and beam halo. Expected $z$ vertex distribution for inelastic collisions, centered at $z=0 \mathrm{~cm}, p$ halo centered at $z=-140 \mathrm{~cm}$, and $\bar{p}$ halo centered at $z=140 \mathrm{~cm}$ (right). . . . . . . . . . . . . . . . . . 14

2.10 Schematic view of the $\mathbf{D} \varnothing$ central tracking system . . . . . 15

2.11 Schematic 3D view of the silicon vertex detector . . . . . . 15

$2.12 \mathrm{XY}$-view of the SMT barrel structure with four super-layers. 16

2.13 a) Location of the CFT. b) Close up view of axial and stereo layers . . . . . . . . . . . . . . . . . . 18

$2.14 y-z$ view of the $\mathrm{D} \varnothing$ magnetic field with both the toroid and solenoid magnets at full current. Numbers are in $\mathrm{kG}$ $(10 k G=1 T) \ldots \ldots \ldots \ldots \ldots \ldots$

2.15 Perspective view of the solenoid inside the central calorimeter. . . . . . . . . . . . . . . . . 19 
2.16 Cross section and layout geometry of CPS and FPS scintillator strips. . . . . . . . . . . . . . . . . . 20

2.17 Isometric view of the central and two end calorimeters . . 21

2.18 Schematic view of a portion of the $\mathrm{D} \varnothing$ calorimeters showing the transverse and longitudinal segmentation pattern. The shading pattern indicates groups of cells ganged together for signal readout. The rays mark pseudorapidity intervals from the centre of the detector. . . . . . . . . . . . 22

2.19 Projection of the calorimeter towers in the i

2.20 Schematic view of a calorimeter cell. . . . . . . . . . 25

2.21 Schematic view of the muon system. . . . . . . . . . . 26

2.22 View of the three drift chamber layers of the muon system. 28

2.23 View of the three scintillator layers of the muon system. . 29

$2.24 \mu^{+} \mu^{-}$invariant mass distributions of various meson resonances: $\omega, \phi, J / \Psi, \Psi^{\prime}, \Upsilon$ and the $\mathbf{Z}$ mass. . . . . . . . . 30

2.25 Schematic view of The Forward Proton Detector (FPD). Quadrupole Roman pot detectors are named $\mathbf{P}$ or $\mathbf{A}$ when placed on the $\mathbf{p}$ or $\bar{p}$ side, respectively. Dipole pots, located on the $\bar{p}$ side, are named D. . . . . . . . . . . . . . . . 31

2.26 Overview of the $\mathrm{D} \varnothing$ trigger and data acquisition systems 32

2.27 Schematic view of subdetectors with L1 and L2 trigger elements. Horizontal arrows indicate the direction of dataflow. 33

4.1 Muon $\eta_{\text {det }}$ Distribution . . . . . . . . . . . . . . . . . . . . 42

4.2 Muon $p_{T}$ Distribution . . . . . . . . . . . . . . . . . . . . . 43

4.3 Di-Muon Invariant Mass . . . . . . . . . . . . . . . . . . . 43

4.4 Pseudo-acolinearity Plot, the arrow indicates the cut applied in this variable . . . . . . . . . . . . . . . . . . 44

4.5 Events that pass dca cuts in CFT and SMT . . . . . . . . . 44

4.6 Events that pass isolation cuts . . . . . . . . . . . . 45

4.7 Acolinearity Cut . . . . . . . . . . . . . . . . . . . 46

4.8 Distance Closest Approach Cut . . . . . . . . . . . . . 47

4.9 Di-Muon $p_{T}$ and Rapidity Distribution . . . . . . . . . . . 47

$4.10 \xi$ Reconstructed in the pbar side . . . . . . . . . . . . . 49

$4.11 \xi$ contribution from di-muons in the pbar side . . . . . . 50

4.12 Number of primary vertices vs $\langle\xi\rangle \ldots \ldots . \ldots . \ldots 50$

4.13 Primary vertex distribution - Before and After Diffractive

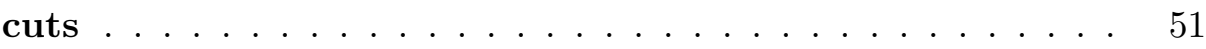

4.14 Fraction of antiproton momentum loss - Gap Requirement applied .............................. 51 
4.15 Energy cell distribution for Electromagnetic cells (Layers 1 to 7 ). Minimum bias, zero bias and emptybx sample. . . 52

4.16 Energy cell distribution for Fine Hadronic cells (Layers 11 to 14). Minimum bias, zero bias and emptybx sample . . . 53

$4.17 \xi$ distributions - Physics signal and Noise for proton and antiproton side . . . . . . . . . . . . . . 53

$4.18 \xi$ distributions - Empty BX (Noise) and MinBias(signal) . 54

4.19 Thresholds for EM and FH calorimeter cells . . . . . . . . 55

$4.20 \xi_{p b a r}$ against $\sum E$ for $-36<i \eta<-32$. Before and After Gap Requirement . . . . . . . . . . . . . 56

4.21 Single Diffractive signal at $\mathbf{D} \emptyset \ldots \ldots \ldots$. . . . . . . 57

$4.22 \xi_{\text {pbar }}$ and Rapidity distributions for Events that pass kinematical cuts . . . . . . . . . . . . . . 57

4.23 Single Diffractive events by POMWIG . . . . . . . . . . . . 58

4.24 Z invariant mass - Pomwig at generated Level . . . . . . . . 59

$4.25 \xi_{\text {pbar }}$ - Pomwig at generated Level . . . . . . . . . . . 60

$4.26 \eta$ and $p_{T}$ distributions for muons from $\mathrm{Z}$ boson - POMWIG at generated level . . . . . . . . . . . . . 60

$4.27 \eta$ distribution for final state particles - POMWIG at generated level. The distribution shows that final particle states destroy the gap of most of diffractive events . . . . . . . . . 61

$4.28 \pi^{ \pm}$Energy distribution in the Gap region - POMWIG at generated level . . . . . . . . . . . . . . 61

$4.29 \xi_{\text {pbar }}$ - Events with two muons into $|\eta|<2$ - Pomwig at generated Level . . . . . . . . . . . . . . . . 62

$4.30 \xi_{\text {pbar }}$ - Geometrical Acceptance - POMWIG at generated Level . . . . . . . . . . . . . . . . . . . 62

$4.31 \xi_{\text {pbar }}$ and Rapidity Distribution - Events that pass all requirements - POMWIG at generated Level . . . . . . . 63

4.32 POMWIG plots after Full Simulation. $P_{t}$ and Rapidity distribution, dimuon mass and $\xi_{\text {pbar }} \ldots \ldots \ldots$. . . . . 64

$4.33 \sum E$ against $\xi_{\text {pbar }}$ - POMWIG events before and after gap requirement ..................... 64

$4.34 \xi_{\text {pbar }}$ and Rapidity distributions for POMWIG events that pass all diffractive cuts . . . . . . . . . . . . . . . 65

4.35 Z kinematical Variables - PYTHIA. $P_{t}$ and Rapidity ditribution, Di-muon mass and $\xi_{\text {pbar }}$ distribution . . . . . . 65

$4.36 \xi_{\text {pbar }}$ and Rapidity distributions for PYTHIA events that pass all diffractive cuts . . . . . . . . . . . . . 66

$4.37 \xi_{\text {pbar }}$ non diffractive cuts DATA and PYTHIA . . . . . 68 
$4.38 \xi_{\text {pbar }}$ Distribution SIGNAL and Background . . . . . . 68

$4.39 \xi_{\text {pbar }}$ Distribution - Diffractive Candidates and POMWIG . 69

$4.40 \Delta \xi$ (Left) and $\xi_{\text {reco }}$ against $\xi_{\text {gen }}$ (Right). The 2D plot is fitted to the polinomial function to ajust the $\xi_{\text {reco }}$ to the $\xi_{\text {gen }} 70$

$4.41 \Delta \xi$ (Left) and $\xi_{\text {corr }}$ against $\xi_{\text {gen }}$ (Right). The 2D plot is fitted to the linear function, the slope is about 1 , it shows the correspondence one by one between both variables . . 71

$4.42 d \sigma / d \xi$ diffractive candidates and POMWIG. The mean value by bin corresponds to the $\xi$ corrected. (See Table 4.6) . . 71

4.43 Rapidity Distribution SIGNAL and Background . . . . . . 72

$4.44 d \sigma / d y$ Diffractive Candidates and POMWIG . . . . . . . 72

C.1 Distribution of $M_{\mu \mu}$ for events exclusively rejected by the isolation cuts. Blue shaded histogram: Expected shape and size of $M_{\mu \mu}$ for signal events rejected by the isolation cuts 83

C.2 Distribution of $M_{\mu \mu}$ for the events exclusively rejected by the requirement that the two muons be oppositely charged. 83

C.3 Distribution of $M_{\mu \mu}$ for events with two isolated muons. The distributions are normalized to the same number of events in the region $M_{\mu \mu}>50 \mathrm{GeV} \ldots \ldots \ldots$. . . . .

C.4 $\Delta t_{A}$ versus $\Delta \alpha_{\mu \mu}$ for events that have passed all cuts but that on $\Delta \alpha_{\mu \mu}$. The red arrow indicates the position of the

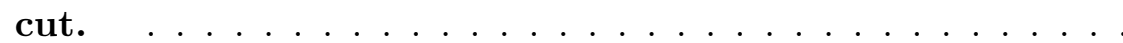

C.5 Speed of muon pair in candidate events as if they were one muon transversing the detector . . . . . . . . . 86

C.6 Distribution of $M_{\mu \mu}$ for the events rejected by the cosmic muons (dca or $\left.\Delta \alpha_{\mu \mu}\right) \ldots \ldots \ldots$

C.7 Distribution of dca for one muon track versus dca for the other muon track for events that fail the dca cut but have passed all other cuts, with the additional requirement that all four isolation cuts pass. (a) For events where both tracks have SMT hits. (b) For events where at least one track has no SMT hits. . . . . . . . . . . . . . . .

D.1 Cube definition surrounding a candidate hot cell in $\eta \times \phi$ plane. . . . . . . . . . . . . . . . . 


\begin{abstract}
Measurements of the inclusive diffractive $Z \rightarrow \mu^{+} \mu^{-}$cross section with gap requirement for $M_{\mu \mu}>40 \mathrm{GeV}$ at $\sqrt{s}=1.96 \mathrm{TeV}$ and fraction of $\mathrm{Z}$ bosons produced diffractively with gap requirement from $\mathrm{Z}$ inclusive production are presented. The measurements are performed using a data sample corresponding to an integrated luminosity of $820 \mathrm{pb}^{-1}$, collected with the $\mathrm{D} \varnothing$ detector at the Tevatron, between 2002 to 2005 . A total of 39945 di-muons events are selected and final results of:

$\sigma_{\text {Diff }}^{\text {gap }} \times \operatorname{Br}\left(Z / \gamma^{*} \rightarrow \mu^{+} \mu^{-}\right)=4.09 \pm 0.64$ (stat. $) \pm 0.88$ (syst. $) \pm 0.27$ (lumi. $) p b$ and,

$$
R_{\text {Diff }}^{\text {gap }}=1.92 \pm 0.30 \text { (stat.) } \pm 0.41 \text { (syst.) } \pm 0.12(\text { lumi }) \%
$$
\end{abstract}

are obtained. In adition, $d \sigma / d \xi$ and $d \sigma / d y$ distributions are presented and they are compared with diffractive montecarlo (POMWIG). A reasonably agreement is obtained in this comparation.

Finally, comparison of fraction of Z bosons produced diffractively with gap requirement (gap fraction) as measured with DØ during Run I of the Tevatron is compared. An good agreement is found for gap fraction results. 


\section{Chapter 1}

\section{Theory}

\subsection{The Standard Model}

Elementary particle physics deals basically with the study of the ultimate constituents of matter and the nature of the interactions between them. It is well known that the everyday life is properly described by Newtons laws of classical mechanics. But for objects that travel at speeds comparable to the speed of light $c$, the classical rules need to be modified by special relativity; furthermore, for objects that are very small (roughly at the subatomic level), classical mechanics is supersede by quantum mechanics. As elementary particles are both fast and small their description falls under the domain of the quantum field theory.

In the present state of our knowledge leptons and quarks are considered to be the elementary constituents of matter Together with the four fundamental interactions (the strong, electromagnetic, weak and gravitational forces) they represent the basic ingredients for a description of the physical world.

The Standard Model describes the interaction between quarks and leptons by means of two mathematical models:Quantum Chromodynamics QCD and the Electroweak Theory [1], [2], [3]. QCD which is based on the SU(3) group, accounts for the strong interaction the Electroweak Theory which successfully unifies the electromagnetic and weak interactions, is based on a group structure of $S U(2)_{L} \times$ $U(1)$.

The fundamental particles considered by the Standard Model are fermions or bosons. The fermions are quarks or leptons with spin $\frac{1}{2}$, that are considered to be the constituents of matter. The bosons have spin and are responsible for mediating the strong and electroweak forces.

The model considers the existence of six electrically charged quarks (Up, Down, 
Charm, Strange, Top and Bottom) paired in three generations as shown in Table 1.1. The existence of all the quarks has been experimentally verified.

\begin{tabular}{|c|c||c|c|c||c|}
\hline Quarks & Charge & Leptons & Charge & Interaction & Gauge Bosons \\
\hline$u, c, t$ & $+\frac{2}{3}$ & $\nu_{e}, \nu_{\mu}, \nu_{\tau}$ & 0 & EM & photon $(\gamma)$ \\
\hline$d, s, b$ & $-\frac{1}{3}$ & $e, \mu, \tau$ & -1 & Weak & $W^{ \pm}, Z^{0}$ \\
\hline$n n n n$ & Strong & gluons $(g)$ \\
\hline
\end{tabular}

Table 1.1: Schematic representation of the Standard Model: Constituents are doublets of quarks and leptons in three generations. Forces are mediated by gauge bosons

Each quark has an additional degree of freedom called color, and labeled Red, Green or Blue. Quarks can only exist in color singlet states and thus can not be isolated. Quarks bound in color singlet states form the hadrons which are found in nature. As the quarks carry electric and color charges, they are subject to both strong and electroweak forces.

The second group of fermions are the leptons which also appear paired in three families (see Table 1.1). The electron $\left(e^{-}\right)$,muon $\left(\mu^{-}\right)$and tau $\left(\tau^{-}\right)$are massive particles which carry a negative electric charge $e=1.6 \times 10^{-19} C$ and thus are subject to electroweak forces. These leptons are paired with three neutrinos which are light possibly massless electrically neutral particles that only experience weak interactions.

The gauge bosons are responsible for mediating the fundamental forces. The coupling constants describing the strength of these forces are all dimensionless.

The strong force which acts between particles carrying color is mediated by eight gluons. Gluons are electrically neutral bosons that carry color charge and therefore undergo self-interactions. The photon $\gamma$ and the three intermediate vector bosons $W^{ \pm}$and $Z^{0}$ mediate the electroweak force. The model also predicts the existence of a neutral scalar Higgs boson which is a remnant of the mechanism that breaks the $S U(2) \times U(1)$ symmetry and generates the $\mathrm{W}$ and $\mathrm{Z}$ boson masses. So far no experimental evidence exists for the Higgs boson.

\section{$1.2 \quad Z^{0}$ Bosons at the Tevatron}

At the Tevatron, $\mathrm{Z}$ bosons are predominantly produced via the leading order annhilation of a quark antiquark pair (the Drell-Yan process [4]): $q \bar{q} \rightarrow Z$. Figure [Fig 1.1] show this process for a $Z$, which will decay to a pair of muons. 


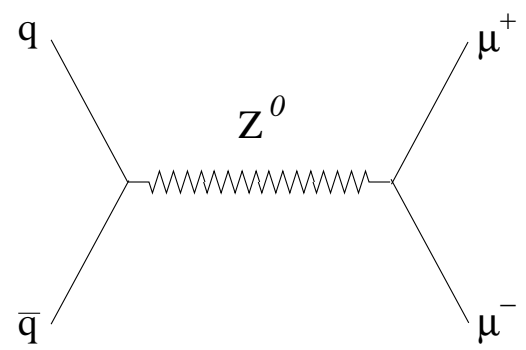

Figure 1.1: $\mathrm{Z}$ production and decay at the Tevatron

The cross section for such processes, $p \bar{p} \rightarrow Z^{0}$, can be written in the factorised form:

$$
\sigma=\sum_{i, j} f_{i}\left(x_{1}, \mu_{F}^{2}\right) \otimes C_{i, j}^{P}\left(x_{1} x_{2} s, \frac{Q^{2}}{\mu_{F}^{2}}\right) \otimes \bar{f}_{j}\left(x_{2}, \mu_{F}^{2}\right),
$$

where $Q^{2}$ is the scale of the hard process, $s$ is the square of the centre of mass energy of the $p \bar{p}$ system, $C_{i, j}^{P}\left(x_{1} x_{2} s, \frac{Q^{2}}{\mu_{F}^{2}}\right)$ are the coefficient functions describing the hard processes, $q_{i} \bar{q}_{j} \rightarrow Z$, and $f_{i}\left(x_{1}, \mu_{F}^{2}\right)$ and $\bar{f}_{j}\left(x_{2}, \mu_{F}^{2}\right)$ are the PDFs of the proton and antiproton respectively. The coefficient functions can be calculated from first principles whereas the PDFs must be taken from fits to experimental data. The partons inside the hadrons that do not participate in the hard interaction will generally interact softly. This part of the process is known as the underlying event.

The bosons are unstable and will decay to a pair of fermions; the branching ratio for the decays [5] are shown in Table 1.2 for the $\mathrm{Z}$ bosons.

\begin{tabular}{|c||c|}
\hline decay channel & branching ratio (\%) \\
\hline$e^{+} e^{-}$ & $3.363 \pm 0.004$ \\
\hline$\mu^{+} \mu^{-}$ & $3.366 \pm 0.007$ \\
\hline$\tau^{+} \tau^{-}$ & $3.370 \pm 0.008$ \\
\hline invisible & $20.00 \pm 0.06$ \\
\hline hadrons & $69.91 \pm 0.06$ \\
\hline
\end{tabular}

Table 1.2: Branching Ratios for $\mathbf{Z}$ boson decays. The invisible decays are presumed to be to neutrinos 


\section{Chapter 2}

\section{Experimental Apparatus}

$\mathrm{D} \varnothing$ is an experiment on a proton-antiproton collider at a center of mass energy of $1.96 \mathrm{TeV}$. This chapter gives an overview of the Tevatron, which produces the collision and the particle detector used to study them.

\subsection{The Fermilab accelerators}

The Tevatron is the final stage in a sequence of seven accelerators [6]. A CockcroftWalton pre-accelerator, a linear accelerator (Linac) and a synchrotron (Booster) provide a source of $8 \mathrm{GeV}$ protons. The antiproton Debuncher and Accumulator are two components of the Antiproton Source. The Main Injector serves as the final boosting stage before injecting protons and antiprotons into the Tevatron. It also provides the necessary source of energetic protons which are needed in the Antiproton Source. Figure Fig 2.1 gives an overview of the Fermilab accelerator complex.

\subsubsection{The Pre-accelerator}

The purpose of the pre-accelerator is to produce negatively charged hydrogen ions $\left(H^{-}\right)$with an energy of $750 \mathrm{keV}$, which are then transferred into the Linac. Hydrogen gas $\left(H_{2}\right)$ enters a magnetron surface-plasma source [Fig 2.2]. Due to the electric field between the anode (negatively charged) and cathode (positively charged), the electrons are stripped away from the hydrogen atoms to create a plasma. The positively charged hydrogen ions then strike the surface of the cathode to collect extra electrons and thereby form negatively charged hydrogen ions. The $\mathrm{H}^{-}$are extracted through the anode aperture with an electric field of $18 \mathrm{kV}$ applied by the extractor plate. 


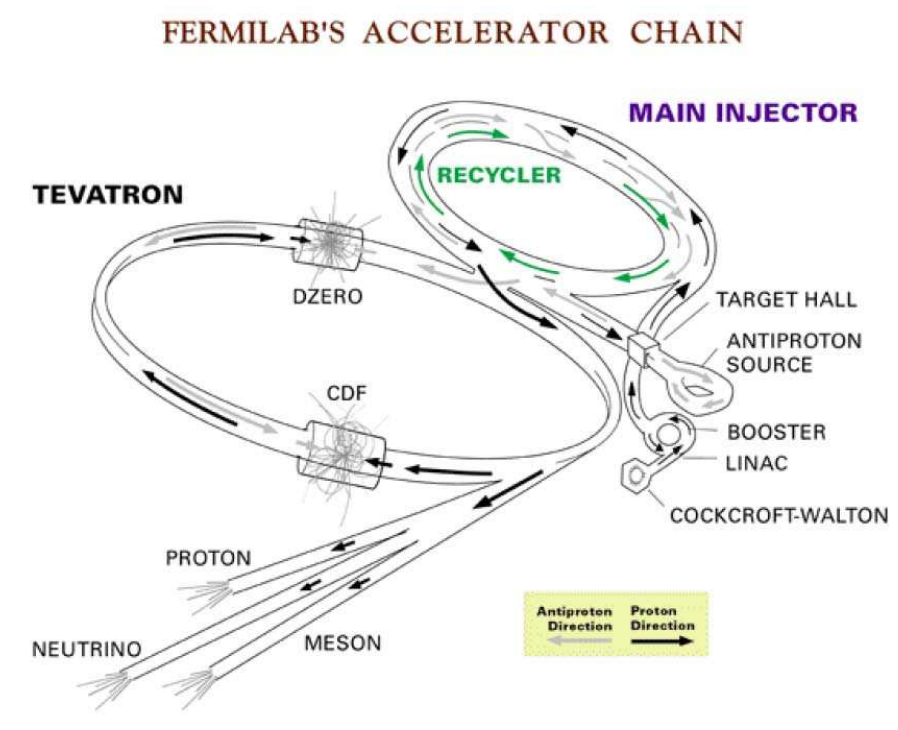

Figure 2.1: The accelerator chain at Fermilab

A commercial Cockcroft-Walton Generator produces a $750 \mathrm{kV}$ potential difference by charging capacitors in parallel from an AC voltage source and discharging them in series, via diodes. The Cockcroft-Walton Generator is used to further accelerate the $\mathrm{H}^{-}$ions to an energy of $750 \mathrm{keV}$. After exiting the Cockcroft-Walton device, the $H^{-}$travel through a transfer line. Before entering the Linac the continuous stream of $H^{-}$ions passes through a single gap radio frequency (RF) cavity which bunches the beam at the RF frequency of the Linac (201.24 MHz).

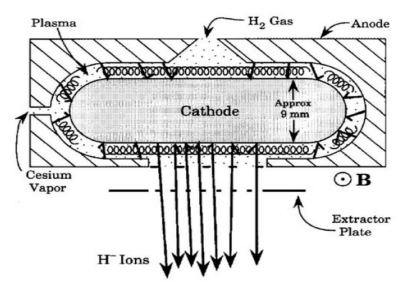

Figure 2.2: Schematic view of magnetron operation for the hydrogen ion source 


\subsubsection{The Linac}

The Linac receives bunches of $750 \mathrm{keV} \mathrm{H}^{-}$ions from the pre-accelerator and accelerates them further to an energy of $400 \mathrm{MeV}$ using RF cavities [Fig 2.3]. The RF cavities are contained within a collection of steel tanks which hold a sequence of drift tubes separated from each other by gaps. In order to accelerate $H^{-}$ions, the cavities are designed in such a way that particles traveling in the gaps experience an acceleration, while particles traveling in the drift tubes are shielded from the RF. After passing through the Linac, bunches of $400 \mathrm{MeV} \mathrm{H}^{-}$ions are transferred into the Booster.

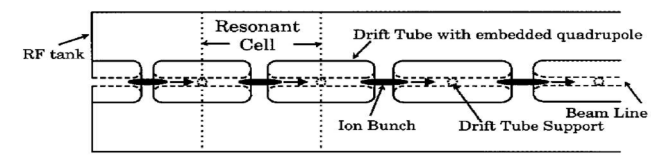

\section{Figure 2.3: Schematic drawing of Linac RF cavity}

\subsubsection{The Booster}

The Booster is the first synchrotron in the chain of accelerators. It consists of a sequence of dipole and quadrupole magnets and $17 \mathrm{RF}$ cavities arranged in a circle with a diameter of $151 \mathrm{~m}$, and accelerates protons to an energy of $8 \mathrm{GeV}$. Negatively charged $H^{-}$ions coming from the Linac are merged with protons $\left(\mathrm{H}^{-}\right)$ ions by letting the combined beams pass through a carbon foil.

Once the Booster is filled with proton bunches, the RF cavities provide an acceleration up to $8 \mathrm{GeV}$. At the same time, the field strength in the dipole magnets is adjusted accordingly in order to maintain a constant radius for the circulating particles. Once the protons have reached an energy of $8 \mathrm{GeV}$, they are transferred into the Main Injector.

\subsubsection{The Main Injector}

The Main Injector is a circular synchrotron with a diameter of $1 \mathrm{~km}$. It can accelerate both protons (coming from the Booster) and antiprotons (coming from the Antiproton Source) from $8 \mathrm{GeV}$ to $150 \mathrm{GeV}$ before injecting them into the Tevatron. It also delivers $120 \mathrm{GeV}$ protons to the Antiproton Source. 


\subsubsection{The Antiproton Source}

The Antiproton Source consists of three major components: the Target Station, the Debuncher, and the Accumulator. In the first step, the Target Station receives 120 $\mathrm{GeV}$ protons from the Main Injector and diverts them onto a Nickel Target. This produces a shower of secondary particles (including antiprotons) at many different angles and with a large spread in particle momentum. A Lithium lens and bending magnets are used to focus the beam and remove positively charged particles [Fig 2.4]. A process called stochastic cooling is used in both the Debuncher and the Accumulator to reduce the spread in momentum and position of the antiprotons, thereby "cooling" them.

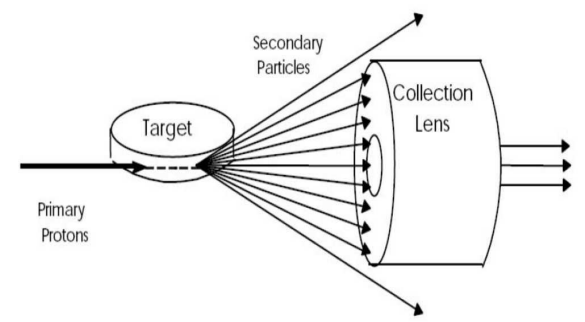

Figure 2.4: Simplified drawing of anti-proton production with nickel target and lithium lens.

Both the Debuncher and Accumulator are located in a rounded-triangle shaped tunnel with a circumference of about $51 \mathrm{~m}$. Antiprotons coming from the Target Station are transferred into the Debuncher where the momentum spread of the particles is reduced. It is technically very challenging to accumulate a large quantity of antiprotons. On average, for every 1 million protons that hit the Nickel target, only about 20 antiprotons can be gathered. Therefore the Accumulator stores antiprotons until a sufficient amount has been generated to be transferred into the Main Injector. The Accumulator must be capable of storing antiprotons over many hours.

\subsubsection{The Tevatron}

The Tevatron is the final stage in the sequence of proton and antiproton acceleration. It has a radius of $1 \mathrm{~km}$ and uses superconducting magnets which operate at liquid helium temperature providing magnetic fields of up to 4 Tesla. Protons and antiprotons are accelerated to $980 \mathrm{GeV}$, leading to a center-of-mass collision energy of $1.96 \mathrm{TeV}$. 
Protons and antiprotons travel in groups of particles (bunches) in opposite directions while sharing the same beam pipe. A full revolution (turn) take about $21 \mu \mathrm{s}$. The Tevatron injects 36 bunches of both protons and antiprotons for each store. A three fold symmetry is imposed by separating the 36 bunches into three superbunches. Overall, this leads to a time structure where bunches of protons and antiprotons (live bunch crossings or zero bias events) collide at $1.7 \mathrm{MHz}$ [7].

Figure [Fig 2.5] shows the integrated luminosity per week and total integrated luminosity for Run II from May 2001 until May 2007.

Collider Run II Integrated Luminosity

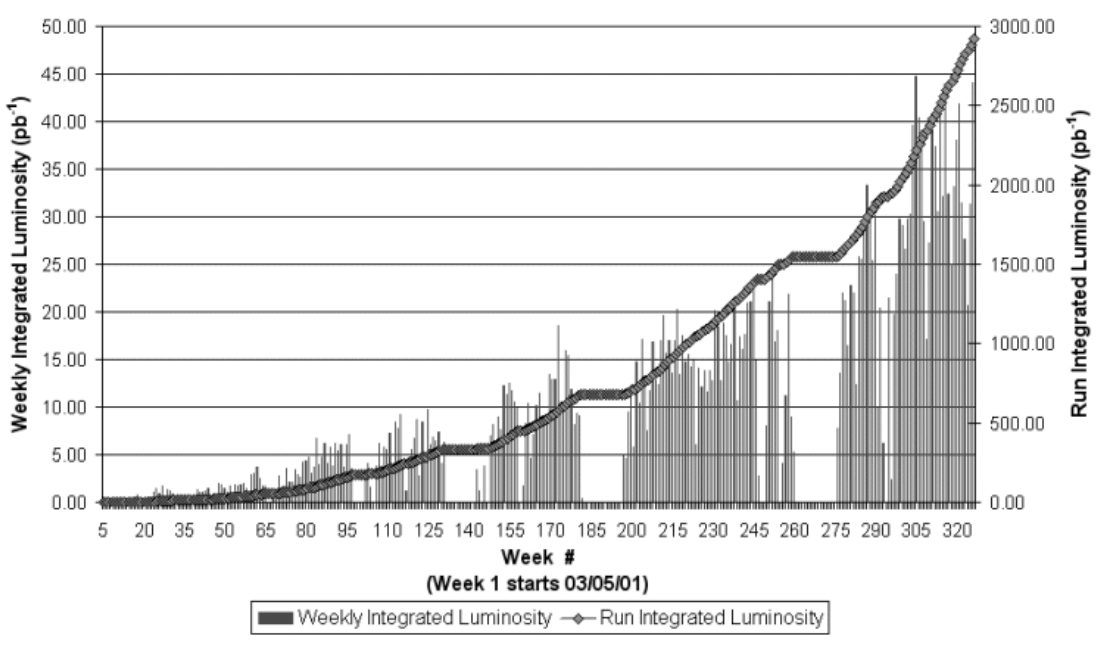

Figure 2.5: The integrated luminosity per week and total integrated luminosity for Run II from May 2001 until May 2007

\subsection{Interactions of Energetic Particles with Matter}

The main method used to detect and measure the properties of particles is to look for the position and energy deposited as those particles pass through a layer of material. In the $\mathrm{D} \varnothing$ detector the relevant particles to analyze are electrons, muons, hadronic particles and neutrinos. The interaction of these particles with detector subsystems results in energy loss which can be detected and measured. Tracking detectors are designed to measure the particle positions with minimal 
energy loss. Calorimeters are constructed to fully absorb the particles and their showers, and thus their energy, in the process of measurement. The interactions of the different types of particles with the $D \varnothing$ detector are described below.

\subsubsection{Electrons and Photons}

Electrons passing through matter loose energy primarily through ionization and bremsstrahlung. Bremsstrahlung is the dominant process, the critical energy equation is

$$
E_{C}=\frac{800}{Z+1.2} \mathrm{MeV}
$$

$\mathrm{Z}$ corresponds to the atomic number of the medium

The emitted photons produce electron-positron pairs, which in turn emit photons. The resulting shower of electrons and photons grows until the energy of the electrons falls below the critical energy. They subsequently interact primarily through ionization. The mean distance over which an electron loses all but $1 / e$ of its energy is called the radiation length $X_{0} \quad[9]$

$$
X_{0}=\frac{716.4 A}{Z(Z+1) \ln (287 / \sqrt{Z})} \mathrm{g} \cdot \mathrm{cm}^{-2},
$$

where $\mathrm{A}$ is the atomic mass of the medium in $g \cdot \mathrm{mol}^{-1}$. Photons interacting with matter produce electron-positron pairs, and hence an electromagnetic shower.

\subsubsection{Muons}

Muons interact through bremsstrahlung at a much lower rate than electrons due to their larger mass. Their energy loss is primarily through ionization. Figure [Fig $2.6]$ shows the energy loss per unit of material for muons in various energy regimes [9]. Muons at the Tevatron have energies of the order of $\mathrm{GeV}$, and hence are minimum ionizing particles, also called MIP. They deposit only minimal energy in the detector and leave it essentially unperturbed, in contrast to all other particles (with the exception of neutrinos).

\subsubsection{Hadronic Particles}

These particles interact inelastically with the nuclei of the detector elements, producing primarily pions and nucleons. At high energies, the resulting particles interact similarly with nearby nuclei, producing a shower of hadronic particles. 


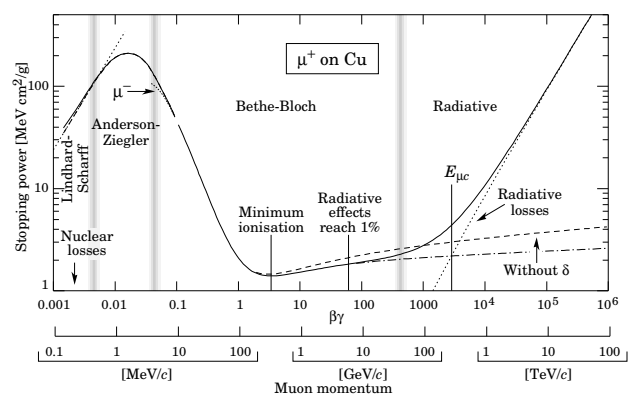

Figure 2.6: Energy loss through ionization of muons in various energy regimes

The characteristic length scale is the nuclear interaction length, which depends on the material density and atomic mass and is roughly independent of energy

$$
\lambda_{I} \approx 35 A^{1 / 3} \mathrm{~g} \cdot \mathrm{cm}^{2}
$$

A significant fraction of energy of the initial hadron escapes the hadronic cascade in form of neutral pions, which produce a secondary cascade. A smaller fraction results in invisible energy lost through unbinding of nuclei by spallation, non-ionizing collisions and uncaptured energy of neutrinos.

\subsubsection{Neutrinos}

Being uncharged leptons, neutrinos interact only weakly via $\mathrm{W}$ and $\mathrm{Z}$ boson exchange, making their energy loss negligible and their direct detection impossible at $\mathrm{D} \varnothing$. Their presence can be inferred, however, from transverse momentum conservation requirements.

\subsection{The DØ Detector}

The DØ detector [8] is a large multi-purpose detector designed to identify and to precisely measure the four-momenta particles. It consists of three major subsystems. At the core of the detector, a magnetized tracking system records precisely the angles of charged particles and measures their transverse momenta. A hermetic, finely grained Uranium and Liquid Argon calorimeter measures the energy

of electromagnetic and hadronic showers. A muon spectrometer measures the momenta of muons. 
Between Run I and Run II the D $\varnothing$ detector was substantially upgraded. A $2 T$ solenoidal magnet has been added to the central tracking detectors, and a silicon microstrip tracker was installed. The forward muon system was also substantially upgraded, providing more robust detectors and enhanced triggering capability. Figure [Fig 2.7] shows an overview of the detector.

The following subsections is a brief overview of the Run II DØ detector.

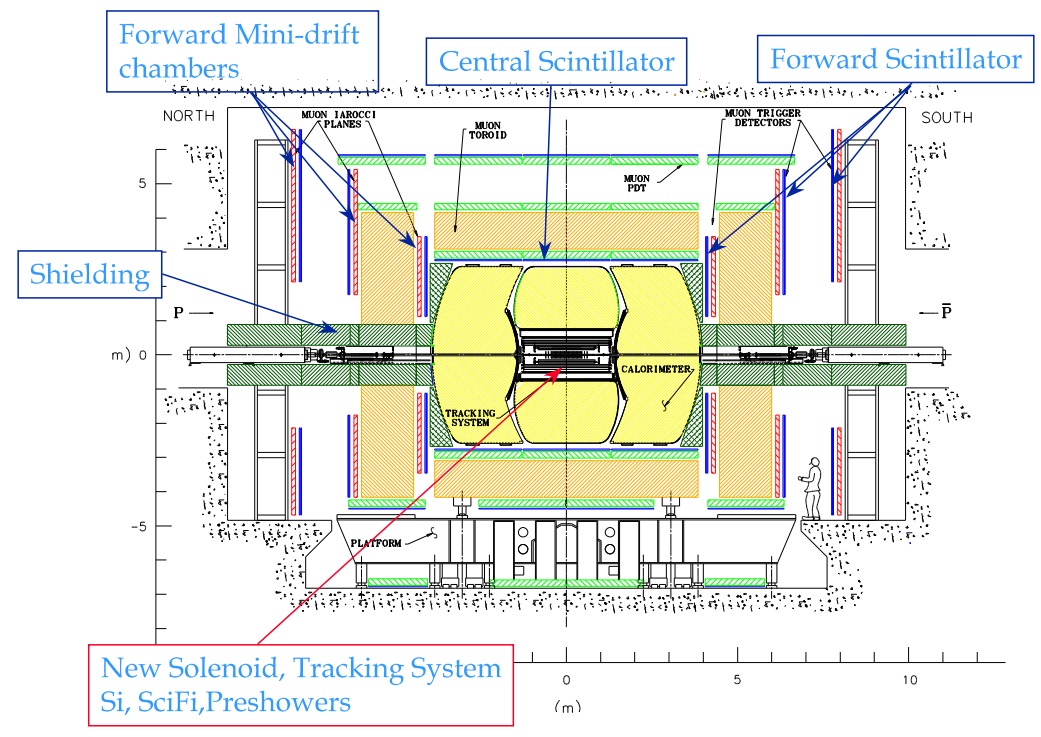

Figure 2.7: Side view of the DØ detector

\subsubsection{Coordinate System}

The cartesian coordinate system used for the $\mathrm{D} \varnothing$ detector is right-handed with the $z$ axis parallel to the direction of the beam such that the protons go in the positive $z$ direction. The $y$ axis is then vertical, and the $x$ axis points towards the center of the accelerator ring.

Another useful set of coordinates are the standard polar coordinates $(r, \phi)$. The coordinate $r$ denotes the perpendicular distance from the $z$ axis,

$$
r=\sqrt{x^{2}+y^{2}}
$$

and $\phi$ is the azimuthal angle 


$$
\phi=\arctan \frac{y}{x},
$$

In addition, a particular reformulation of the polar angle,

$$
\theta=\arccos \left(\frac{z}{\sqrt{x^{2}+y^{2}+z^{2}}}\right)
$$

is given by the pseudo-rapidity $\eta$, defined as:

$$
\eta=-\ln \tan \left(\frac{\theta}{2}\right)
$$

The pseudo-rapidity, obtained from the rapidity $y=\frac{1}{2} \ln \left(\frac{E+p_{z}}{E-p_{z}}\right)$ when particle masses are neglected, is a convenient choice at a hadron collider as the multiplicity of high energy particles is roughly constant in $\eta$. In addition, rapidity intervals are Lorentz-invariant under boosts along the $z$ axis.

Depending on the choice of the origin of the coordinate system, the coordinates are referred to as physics coordinates when the origin is the reconstructed vertex of the interaction $(\phi$ and $\eta$ ), and they are referred to as detector coordinates $\left(\phi_{\text {det }}\right.$ and $\left.\eta_{\text {det }}\right)$ when the origin is chosen to be the center of the DØ detector.

In many cases some of the products of a proton-antiproton collisions escape down the beampipe, which makes it difficult to measure momentum components along the z-axis accurately. In addition, the initial longitudinal momentum of the collision is not known, as the colliding partons carry only a fraction of the proton or the antiproton momentum. Therefore it is more convenient to use the momentum vector projected onto a plane perpendicular to the beam axis (transverse momentum)

$$
p_{T}=p \cdot \sin \theta
$$

In a similar fashion transverse energy is defined as

$$
E_{T}=E \cdot \sin \theta
$$

Usually, the four-momentum vectors for objects observed in the calorimeter are calculated using energies measured by the calorimeter. 


\subsubsection{Luminosity Monitor}

The main purpose of the Luminosity Monitor (LM) [10] is to make an accurate determination of the Tevatron collider luminosity at the $\mathrm{D} \varnothing$ interaction region [11]. It consists of two arrays of twenty-four plastic scintillation counters with photomultiplier readout. A schematic drawing of the system is shown [Fig 2.8].
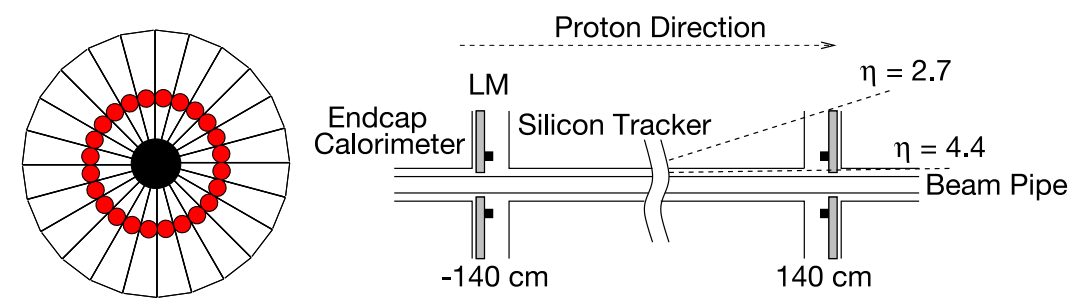

Figure 2.8: Luminosity Monitor layout. The $r-\phi$ view is shown on the left, the $r-z$ view of the two arrays is shown on the right.

The arrays are located in front of the end calorimeters at $z= \pm 140 \mathrm{~cm}$, and occupy the region between the beam pipe and the Forward Preshower Detector (FPS). The counters are $15 \mathrm{~cm}$ long and cover the pseudorapidity range $2.7<$ $\left|\eta_{\text {det }}\right|<4.4$. The luminosity is measured by detecting inelastic $p \bar{p}$ collisions in the Luminosity Monitor, and determined as

$$
\mathcal{L}=\frac{R}{\varepsilon A \sigma_{\text {inelastic }}},
$$

$R$ is the event rate. $\sigma_{\text {inelastic }}$ is the total inelastic cross section [63]. The event rate has to be corrected for the efficiency $\varepsilon$ and the acceptance $A$ of the LM dtector for inelastic $p \bar{p}$ collisions.

Multiple $p \bar{p}$ collisions can occur in a single beam crossing. The number of interactions per bunch crossing is described by Poisson statistics. Collision products arrive at each set of scintillators roughly in coincidence, while beam halo products passing through the detector appear distinctly separated. Time-of-light information from the two luminosity arrays and the $z$ vertex distribution (see Fig 2.9) is utilized to separate these processes. The rate $R$ is corrected for these two efects.

\subsubsection{The Central Tracking System}

The purpose of the central tracking system is to measure the momentum, direction and the sign of the electric charge for particles produced in a collision. It is 

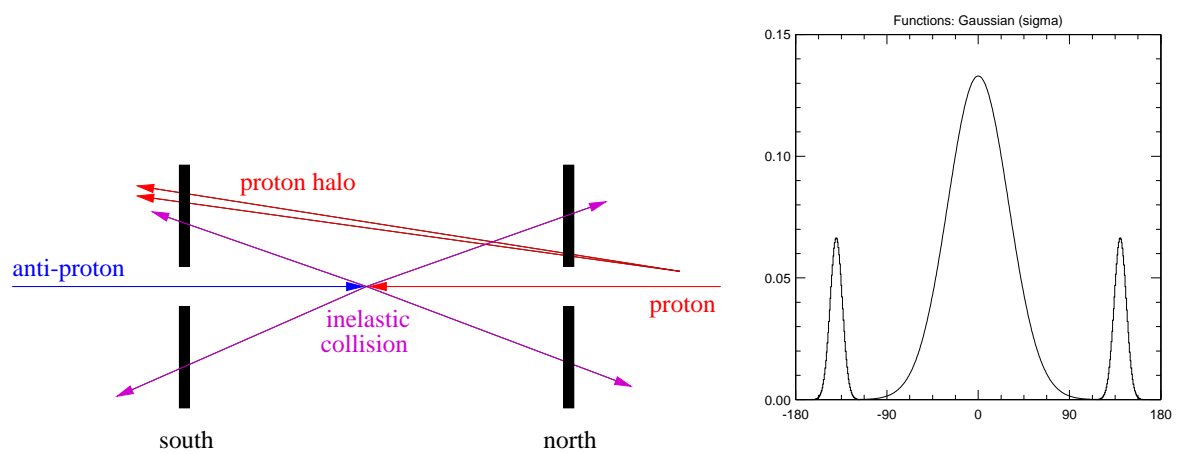

Figure 2.9: The sketch on the left shows the diferentiation between inelastic collisions and beam halo. Expected $z$ vertex distribution for inelastic collisions, centered at $z=0 \mathrm{~cm}, p$ halo centered at $z=-140 \mathrm{~cm}$, and $\bar{p}$ halo centered at $z=140 \mathrm{~cm}$ (right).

surrounded by a solenoid which provides a nearly uniform magnetic field of $B=2 T$ parallel to the beam axis. Charged particles produced in a collision are bent around the field lines. The radius $r$ of the curvature allows the measurement of the transverse momentum through

$$
p_{T}=0.3 \cdot r \cdot B
$$

$p_{T}$ is measured in $\mathrm{GeV}$, the radius $r$ is measured in meters and the magnetic field $B$ in Teslas (T).

The track direction in the $r-z$ plane completes the measurement of the threedimensional momentum vector of the particle.

Closest to the beam pipe is the Silicon Microstrip Tracker (SMT), which allows for an accurate determination of impact parameters and identication of secondary vertices. Surrounding the SMT is the Central Fiber Tracker (CFT), composed of 16 layers of scintillating fiber. The CFT extends to a radius of $50 \mathrm{~cm}$, giving a lever arm long enough to provide efective transverse momentum resolution. The DØ central tracking system is shown in Figure [Fig 2.10].

\section{The Silicon Microstrip Tracker (SMT)}

The Silicon Microstrip Tracker (SMT) is the innermost system in the D $\varnothing$ detector [8]. 


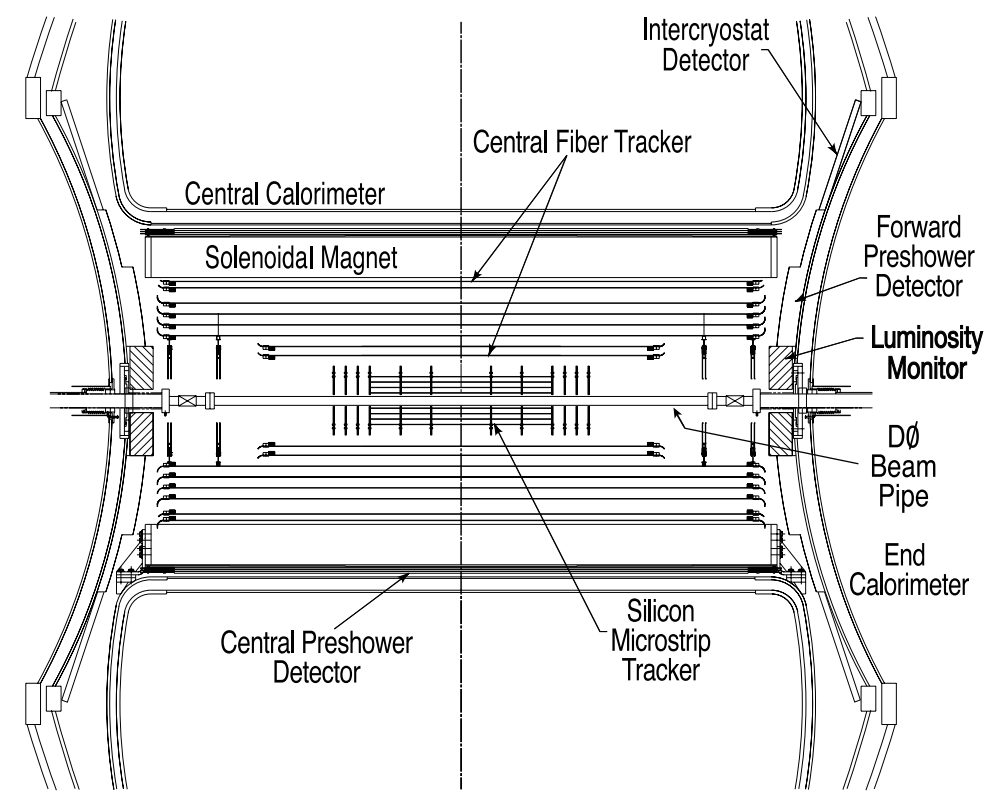

Figure 2.10: Schematic view of the DØ central tracking system

The length of the interaction region $(\sigma \approx 25 \mathrm{~cm})$ sets the length scale of the device in $z$. With an extended interaction region, it is difficult to deploy detectors such that the tracks are generally perpendicular to detector surfaces for all $\eta$. This led to the design of barrel modules interspersed with disks in the center, and assemblies of disks in the forward and backward regions. The barrel detectors measure primarily the $r-\phi$ coordinate, and the disk detectors measure $r-z$ as well as $r-\phi$ coordinate, and the disk detectors measure $r-z$ as well as $r-\phi$ Thus vertices for high $\eta$ particles are reconstructed in three dimensions by the disks, and vertices of particles at small values of $\eta$ are measured in the barrel. Figure[Fig 2.11] shows the Silicon Microstrip Tracker.

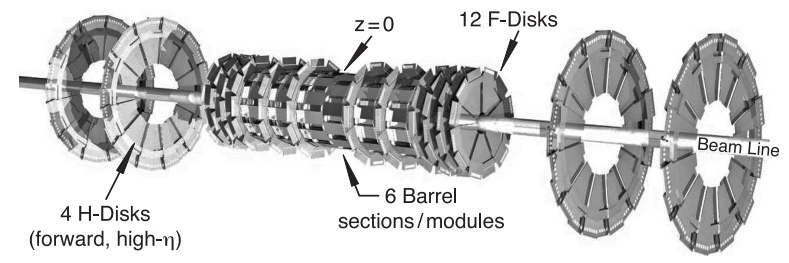

Figure 2.11: Schematic 3D view of the silicon vertex detector 
The detector has six barrels in the central region. Each barrel has four silicon readout layers, each layer having two staggered and overlapping sub-layers, as shown in Figure [Fig 2.12].

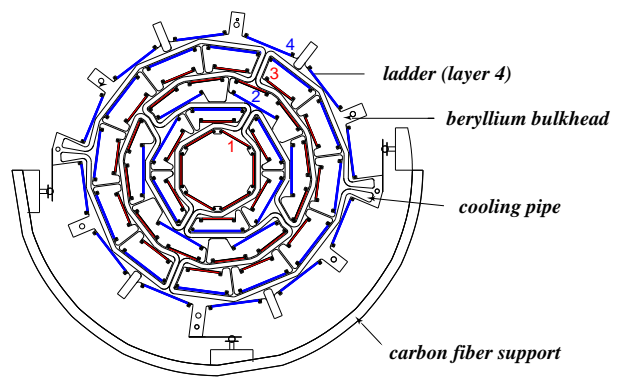

Figure 2.12: XY-view of the SMT barrel structure with four super-layers.

The outer barrels have single sided and double sided $2^{\circ}$ stereo ladders. The four inner barrels have double sided $90^{\circ}$ stereo and double sided $2^{\circ}$ stereo ladders. Each barrel is capped at high $|z|$ with a disk of twelve double sided wedge detectors, called an "F-disk". In the far forward and backward regions, a unit consisting of three F-disks and two large-diameter "H-disks" provides tracking at high $\left|\eta_{\text {det }}\right|<$ 3.0. The H-disks are made of 24 pairs of single sided detectors glued back to back. Table 2.1 lists some specifications of the SMT [12].

\begin{tabular}{cccc}
\hline & Barrels & F-Disks & H-Disks \\
\hline \# Channels & 387072 & 258048 & 147456 \\
Sensors & s/d-sided & double-sided & single-sided \\
Stereo & $0^{\circ}, 2^{\circ}, 90^{\circ}$ & $\pm 15^{\circ}$ & $\pm 7.5^{\circ}$ \\
\# Modules & 432 & 144 & 96 pairs \\
Si area & $1.3 \mathrm{~m}^{2}$ & $0.4 \mathrm{~m}^{2}$ & $1.3 \mathrm{~m}^{2}$ \\
Inner radius & $2.7 \mathrm{~cm}$ & $2.6 \mathrm{~cm}$ & $9.5 \mathrm{~cm}$ \\
Outer radius & $9.4 \mathrm{~cm}$ & $10.5 \mathrm{~cm}$ & $26 \mathrm{~cm}$ \\
Maximal $|z|$ & $38.4 \mathrm{~cm}$ & $54.8 \mathrm{~cm}$ & $120 \mathrm{~cm}$ \\
\hline
\end{tabular}

Table 2.1: Specifications of the Silicon Vertex Detector

Charged particles passing through the $300 \mu m$ thick wafers of $n$-type silicon which conform the SMT produce pairs of electrons and holes. The ionized charge is collected by strips of $p$-type $n^{+}$-type silicon, whose separation (between $\sim 50 \mu \mathrm{m}$ and $\sim 150 \mu \mathrm{m}$ pitch) provide for the measurement of the hits position. The axial 
hit resolution is of the order of $10 \mu \mathrm{m}$, the $z$ hit resolution is $35 \mu \mathrm{m}$ for $90^{\circ}$ stereo and $450 \mu \mathrm{m}$ for $2^{\circ}$ stereo ladders.

\section{Central Fiber Tracker (CFT)}

The scintillating fiber detector, (CFT) [13] consist of $835 \mu \mathrm{m}$ scintillating fibers mounted on eight concentric support cylinders and occupies the radial space from 20 to $52 \mathrm{~cm}$ from the center of the beam pipe. The two innermost cylinders are $1.66 \mathrm{~m}$ long, and the outer six cylinders are $2.52 \mathrm{~m}$ long. Each cylinder supports one doublet layer of fibers oriented along the beam direction and a second doublet layer at a stereo angle of alternating $+3^{\circ}$ and $-3^{\circ}$. The two layers of fibers are offset by half a fiber width to provide improved coverage. The small fiber diameter gives the CFT a cluster resolution of about $100 \mu \mathrm{m}$ per doublet layer.

Light production in the fibers is a multistep process. When a charged particle traverses one of the fibers, the scintillator emits light at $\lambda=340 \mathrm{~nm}$ through a rapid fluorescence decay. A wave-shifting dye absorbs the light at $\lambda=340 \mathrm{~nm}$ and emits it at $\lambda=530 \mathrm{~nm}$. The light is then transmitted by total internal reflexion to the end of the scintillating fibers, where the light is transfered through an optical connection to clear ber waveguides of identical diameter which are 7.8 to $11.9 \mathrm{~m}$ long. The light is only observed from one end of each scintillating fiber. The opposite end of each of the scintillating fibers is mirrored by sputtering with an aluminum coating that provides a reflectivity of 85 to $90 \%$. The clear fiber waveguides carry the scintillation light to visible light photon counters (VLPCs), which convert it to an electronic pulse which is read out.

The visible light photon counters are situated in a liquid Helium cryostat and operate at a temperature of $9 \mathrm{~K}$. They detect photons with a quantum efficiency of $85 \%$ and provide charge of about 30 to $60 k$ electrons per photon. A minimum ionizing particle creates an average of eight photo-electrons per layer, depending on the angle between the scintillating fiber and the particle trajectory. [Fig 2.13] shows a schematic view of the CFT.

\subsubsection{Solenoid Magnet}

The momenta of charged particles are determined from their curvature in the 2 Tesla magnetic field (See Fig 2.14) provided by a $2.7 \mathrm{~m}$ long superconducting solenoid magnet [14].

The superconducting solenoid, a two layer coil with mean radius of $60 \mathrm{~cm}$, has a stored energy of $5 \mathrm{MJ}$ and operates at $10 \mathrm{~K}$. Inside the tracking volume, the magnetic field along the trajectory of any particle reaching the solenoid is uniform within $0.5 \%$. The uniformity is achieved in the absence of a field-shaping iron 


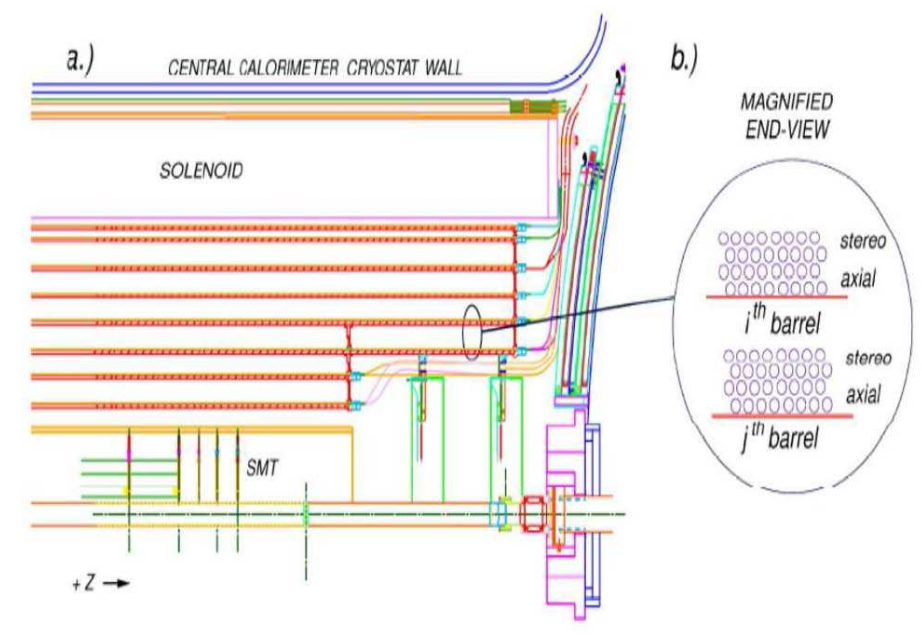

Figure 2.13: a) Location of the CFT. b) Close up view of axial and stereo layers

return yoke by using two grades of conductor. The superconducting solenoid coil

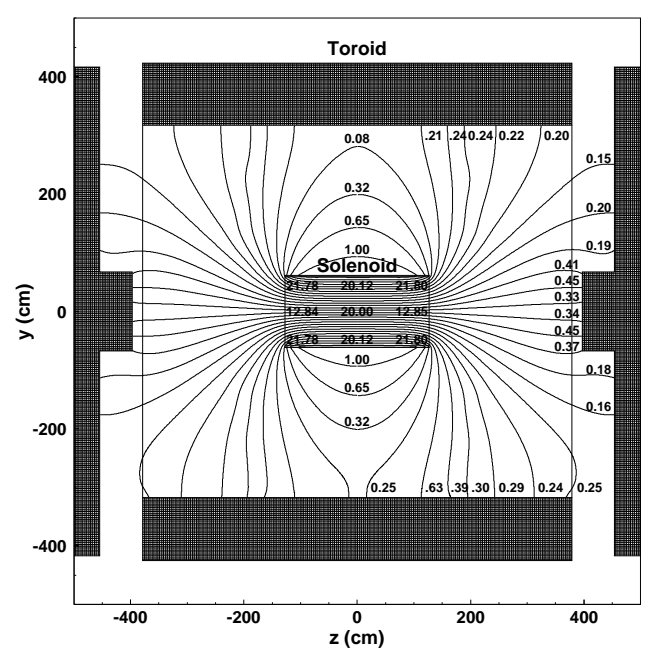

Figure 2.14: $y-z$ view of the $\mathbf{D} \varnothing$ magnetic field with both the toroid and solenoid magnets at full current. Numbers are in kG $(10 k G=1 T)$ 
plus cryostat wall has a thickness of about 0.9 radiation lengths. Figure [Fig 2.15] shows a perspective view of the solenoid inside the central calorimeter with its chimney and control dewar.

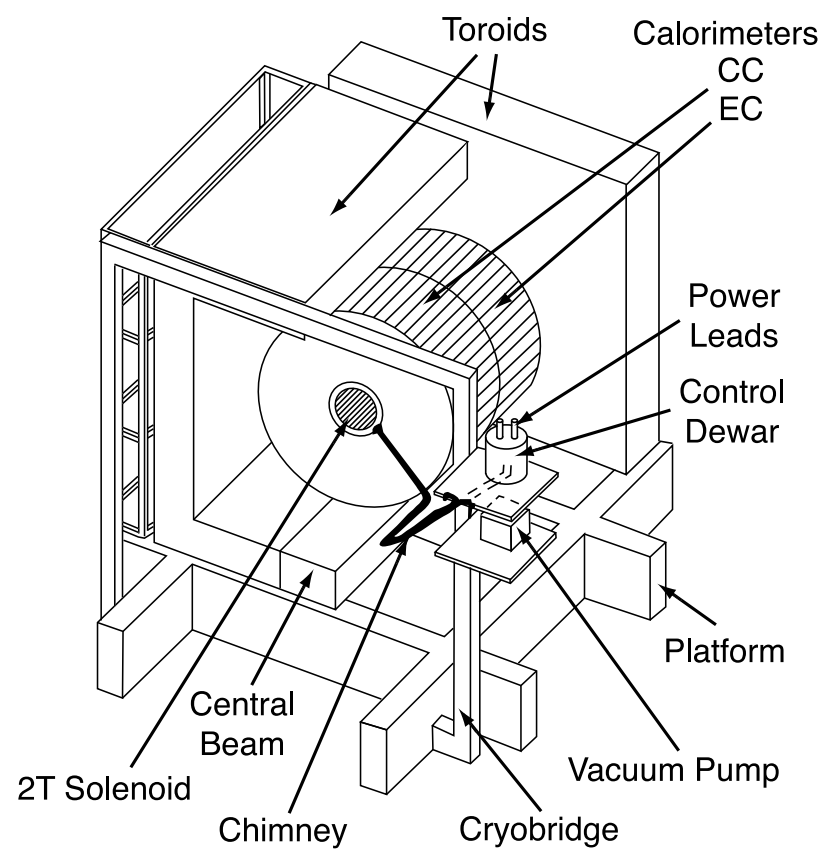

Figure 2.15: Perspective view of the solenoid inside the central calorimeter.

\subsubsection{Momentum Resolution}

Hits from both tracking detectors are combined to reconstruct tracks. The momentum resolution of the tracker for minimum ionizing particles can be parameterized as

$$
\sigma\left(p^{-1}\right)=\frac{\sqrt{(S \cdot \sqrt{\cosh \eta})^{2}+\left(C \cdot p_{T}\right)^{2}}}{p}
$$

where $p$ is the particle momentum and $\eta$ is the pseudo-rapidity. S accounts for the multiple scattering term and $\mathrm{C}$ represents the resolution term. A study [15] of $Z \rightarrow \mu^{+} \mu^{-}$events has found $S=0.015$ and $C=0.0018$.

The two tracking detectors are together capable of locating the primary interaction vertex with a resolution of $35 \mu \mathrm{m}$ along the beam line. They can tag 
b-quark jets with an impact parameter resolution better than $15 \mu \mathrm{m}$ in $r-\phi$ for particles with transverse momentum greater than $10 \mathrm{GeV}$ at $|\eta|=0$.

\subsubsection{Pre-shower Detectors}

The pre-shower detectors [16] use a mixture of tracking and calorimetry. The basic principle is to introduce some material before the pre-shower detectors to induce electromagnetic, but not hadronic, shower formation. Then, with sufficient detector resolution, it is possible to separate electromagnetic objects from hadrons.

Preshower scintillators are triangular shaped [Fig 2.16]. This arranges scintillator layers without creating any dead space and thereby improves the accuracy of position measurements. The center of each scintillator carries a wavelengthshifting fiber which collects the light created by passing charged particles. The light is transmitted via clear fibers to VLPCs for readout.

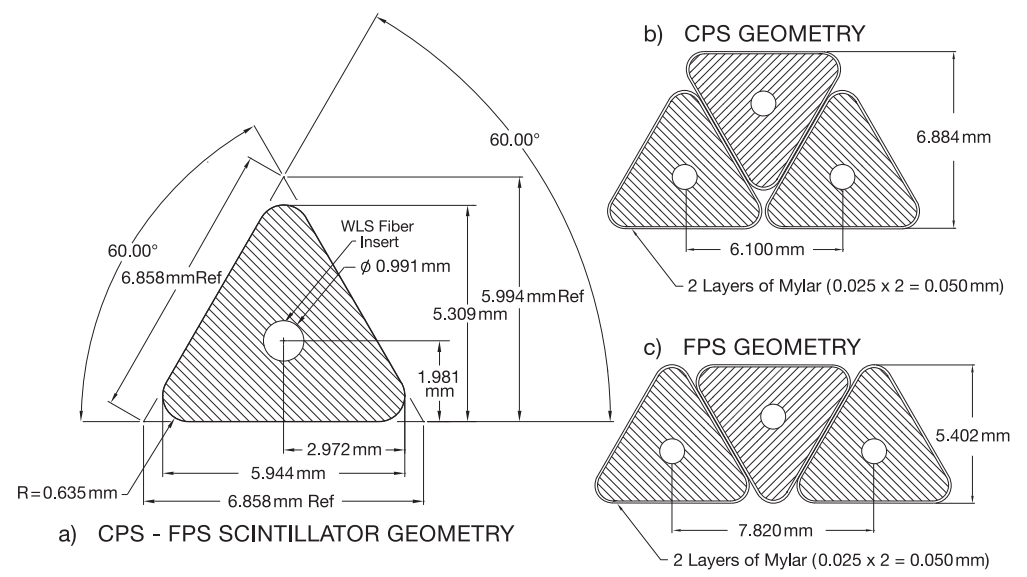

Figure 2.16: Cross section and layout geometry of CPS and FPS scintillator strips.

The Central Preshower Detector (CPS) is located in the $5 \mathrm{~cm}$ gap between the solenoid and the central calorimeter, covering the region $|\eta|<1.3$ (See Figure[Fig 2.10]). It consist of a layer of lead radiator which has a thickness corresponding to approximately one radiation-length $\left(X_{0}\right)$, followed by three layers of triangular scintillator strips. The scintillating layers are arranged in an axial- $u-v$ geometry, with a $u$ stereo angle of $23.8^{\circ}$ and a $v$ stereo angle of $24^{\circ}$. Each layer has a total number of 2560 readout channels.

The two Forward Preshower Detectors (FPS) are attached to the faces of the end calorimeters and cover a region of $1.5<|\eta|<2.5$ (See Figure [Fig 2.10]). 
Each detector consists of an upstream double layer of scintillator strips (minimum ionizing particle layers, or MIP layers), followed by a lead-stainless-steel absorber layer and another double layer of scintillator strips behind it (shower layers).

\subsubsection{The Calorimeter System}

The calorimeter system was designed to measure the energy of electrons, photons, jets and neutrinos by inducing them to produce electromagnetic and hadronic showers. The calorimeter system is segmented longitudinally into electromagnetic and hadronic calorimeters. The electromagnetic calorimeter measures the energy of electrons and photons. The hadronic calorimeter measures the energy of hadrons as they interact with the material of the calorimeter. Muons only deposit a small amount of energy due to ionization. Neutrinos deposit no energy in the detector, but the absence of energy deposition results in a momentum imbalance in the transverse plane. The imbalance is called missing transverse energy $\left(E_{T}\right)$.

The calorimeter consists of a central calorimeter (CC) covering roughly $\left|\eta_{\text {det }}\right|<$ 1 , two end calorimeters (EC) extending the coverage to $\left|\eta_{\text {det }}\right| \approx 5$ and an intercryostat detector (ICD). These parts are shown in [Fig 2.17].

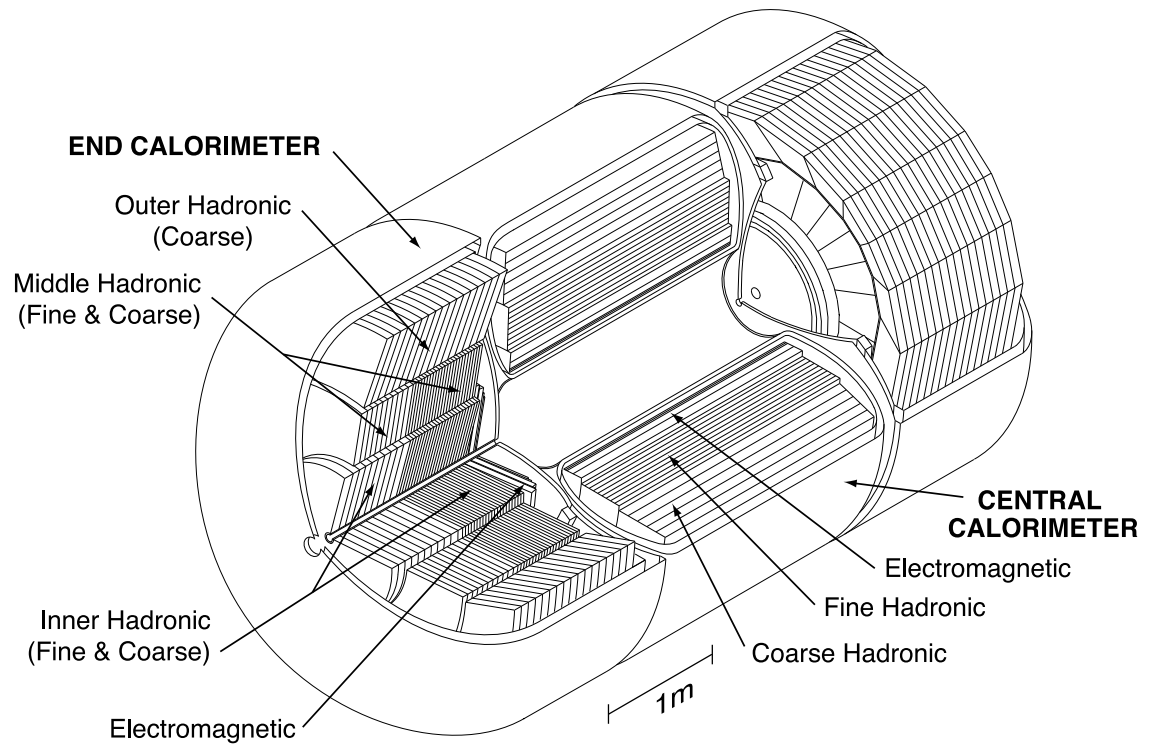

Figure 2.17: Isometric view of the central and two end calorimeters

The central and end calorimeters are subdivided into three layers: the electromagnetic (EM) layer which is designed to measure electrons, positrons and 
photons, known collectively as electromagnetic (EM) objects, the electromagnetic section has 4 layers. The fine $(\mathrm{FH})$ and coarse $(\mathrm{CH})$ hadronic layers measure hadronic showers of particles. Fine hadronic calorimeter $(\mathrm{FH})$ has 2 to 4 layers, and the coarse hadronic section $(\mathrm{CH})$ has 1 to 3 layers.

Each layer is divided into small units called cells. The readout cells are arranged and sized such that each covers roughly an area of $\Delta \eta \times \Delta \phi=0.1 \times 0.1$, comparable to the transverse sizes of showers: $1-2 \mathrm{~cm}$ for EM showers and about $10 \mathrm{~cm}$ for hadronic showers. This geometry, with towers of cells radiating from the centre of the detector, is known as pseudo-projective because the cells lie along lines of pseudorapidity but their boundaries do not. Figure [Fig 2.18] shows the $\eta$ distribution in the calorimeter.

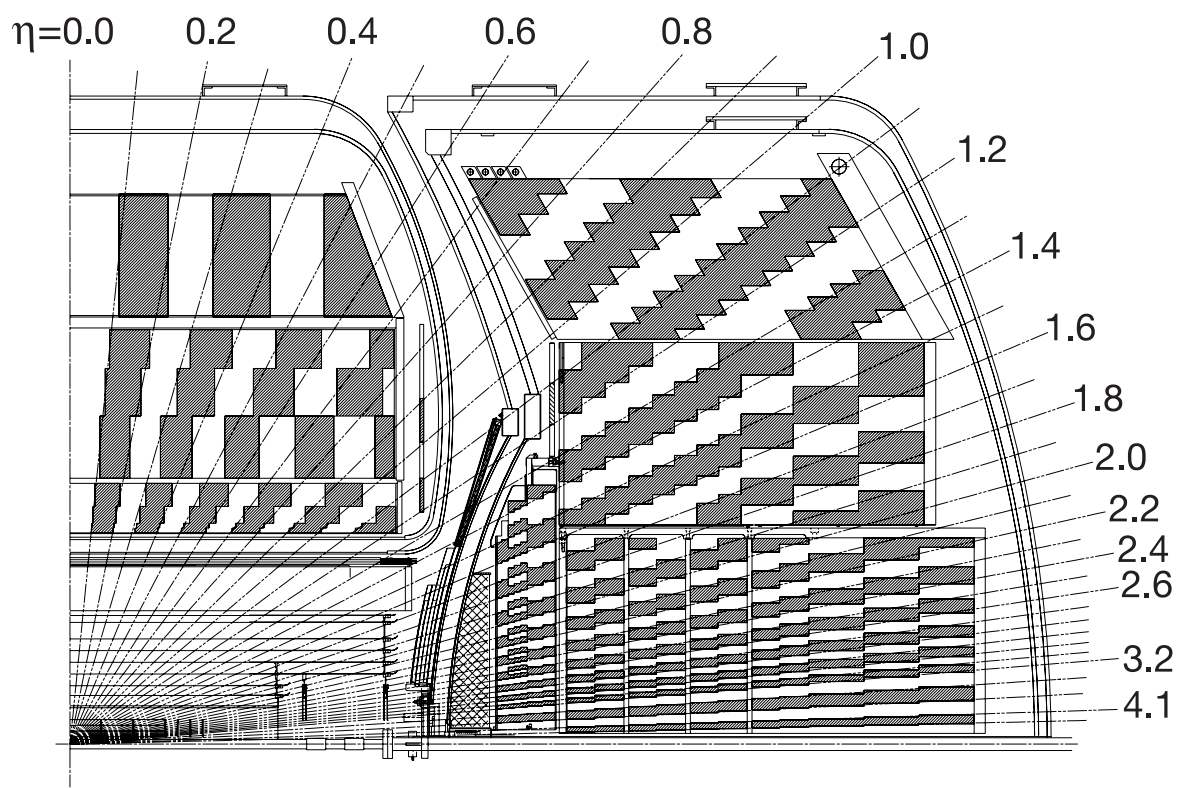

Figure 2.18: Schematic view of a portion of the $\mathbf{D} \varnothing$ calorimeters showing the transverse and longitudinal segmentation pattern. The shading pattern indicates groups of cells ganged together for signal readout. The rays mark pseudorapidity intervals from the centre of the detector.

The calorimeter cells have an unique detector coordinates: layer, $i \eta, i \phi$. The layers 1 to 7 correspond to the Electomagnetic calorimeter, layers from 11 to 14 correspond to the hadronic calorimeters ( $\mathrm{FH}$ and $\mathrm{CH}) . i \eta$ and $i \phi$ are integers that 
correspond to location in $\eta$ and $\phi$. Each $i \eta$ value corresponds to detector $\eta$ with a width of $\Delta \eta=0.1$. The $i \eta$ range is -37 to 37 , there is not $i \eta=0$. The $i \phi$ range that correspond to the azimuthal angle $\phi$ is 1 to 64 with a width of $\Delta \phi=2 \pi / 64 \approx 0.1$. There are two regions where the cells have different sizes. In the forward region $|\eta|>3.2$ the cell's width are larger in $\Delta \eta$ because of the diminishing physical size of pseudorapidity units (See Table 2.2), and their azimuthal width doubles to $\Delta \phi=0.2$. Up to $|\eta|<2.6$, the third EM layer is divided more finely with $\Delta \eta \times \Delta \phi=0.05 \times 0.05$. This is to define the EM showers more precisely at the depth of their maximum energy deposition. The EM layers extend to $|\eta|=4.1$, the first two $\mathrm{FH}$ layers to $|\eta|=4.45$, and the last two $\mathrm{FH}$ layers and one $\mathrm{CH}$ layer extend to $|\eta|=5.2$. Figure [Fig 2.19] shows the $i \eta$ range for the layers of the calorimeter.

\begin{tabular}{|c|c|}
\hline$i \eta$ & $|\eta|$ range \\
\hline \hline 1 & $0.0-0.1$ \\
\hline 2 & $0.1-0.2$ \\
\hline$\ldots$ & $\ldots$ \\
\hline 32 & $3.1-3.2$ \\
\hline 33 & $3.2-3.42$ \\
\hline 34 & $3.42-3.7$ \\
\hline 35 & $3.7-4.1$ \\
\hline 36 & $4.1-4.45$ \\
\hline 37 & $4.45-5.2$ \\
\hline
\end{tabular}

Table 2.2: Pseudorapidity coordinate $|i \eta|$ in the calorimeter

The active medium for all of the calorimeters is liquid Argon. Each of the three calorimeters is located within a cryostat that maintains the temperature at approximately $80 \mathrm{~K}$. In order to achieve the same energy response for electromagnetic and hadronic particles $(e / h \approx 1)$, different absorber plates are used in different locations. The EM sections use thin 3 or $4 \mathrm{~mm}$ plates, made from nearly pure depleted Uranium. The fine hadronic sections are made from 6-mm-thick Uranium-Niobium alloy. The coarse hadronic modules contain relatively thick $46.5 \mathrm{~mm}$ plates of Copper in the CC and stainless steel in the EC.

The elements are combined in a basic unit (calorimeter cell, shown in Figure Fig 2.20) which contains the absorber plates, the active medium, and a Copper readout pad laminated to G10 and covered in resistive epoxy for collecting the ionization. The electric field is established by grounding the absorber plates and holding the resistive surface of the pad at typically $1.6 \mathrm{kV}$. The electron drift time 


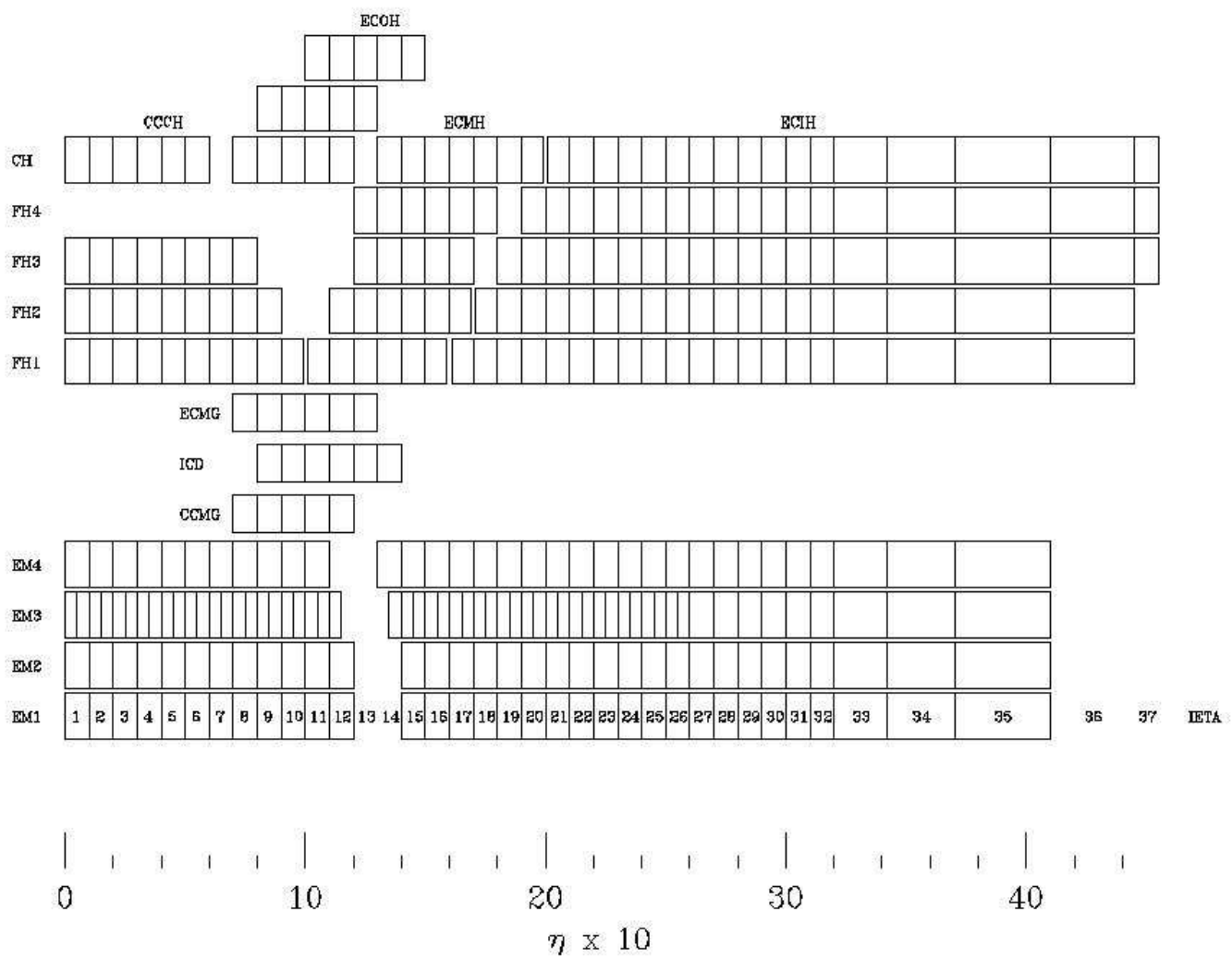

Figure 2.19: Projection of the calorimeter towers in the $i \eta$-layer plane

across the $2.3 \mathrm{~mm}$ gap is approximately $450 \mathrm{~ns}$.

\section{The Inter-Cryostat Detectors}

Given that the calorimeter system is contained in three separate cryostats, it provides incomplete coverage in the pseudorapidity region $0.8<\left|\eta_{\text {det }}\right|<1.4$, as can be seen in Figure [Fig 2.18]. Additional layers of sampling detectors are added in the form of scintillating counters between the CC and EC cryostats. These counters are called Inter-Cryostat Detector or ICD [17]. They have a segmentation of $\Delta \eta \times \Delta \phi=0.1 \times 0.1$, that matches exactly the calorimeter geometry. In addition, separate single-cell scintillator structures, called massless gaps [17] are installed, both in the central calorimeter and in the end cap calorimeters. The ICD allows 


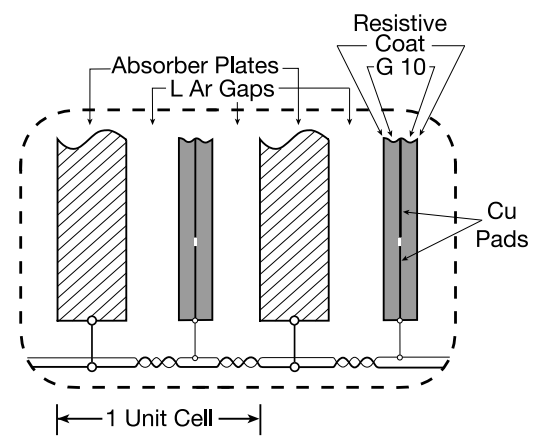

Figure 2.20: Schematic view of a calorimeter cell.

sampling of the region between the Central and End Calorimeters improving the energy resolution.

\section{Calorimeter Resolution}

The energy resolution of the electromagnetic and hadronic calorimeter modules was studied in Run I using pions and electrons from a test beam [18] with energies between 10 and $150 \mathrm{GeV}$, typical energies for production in Tevatron collisions. The relative energy resolution can be parameterized as:

$$
\frac{\sigma(E)}{E}=\sqrt{C^{2}+\left(\frac{S}{\sqrt{E}}\right)^{2}+\left(\frac{N}{E}\right)^{2}} .
$$

The dominant efect in the energy resolution comes from sampling fluctuations which are of Poisson nature and is represented by S. N represents contributions from Uranium and electronics noise, and $\mathrm{C}$ originates from calibration errors and other systematic effects. The results are summarized in Table 2.3.

\begin{tabular}{ccrc}
\hline Particle & $\mathrm{C}$ & $\mathrm{S}$ & $\mathrm{N}$ \\
\hline$e$ & $0.0115_{-0.0036}^{+0.0027}$ & $0.135 \pm 0.005 \sqrt{\mathrm{GeV}}$ & $0.43 \mathrm{GeV}$ \\
\hline$\pi^{ \pm}$ & $0.032 \pm 0.004$ & $0.45 \pm 0.04 \sqrt{\mathrm{GeV}}$ & $0.975 \mathrm{GeV}$ \\
\hline
\end{tabular}

Table 2.3: Calorimeter Energy Resolution Parameters, measured with electrons for the electromagnetic section ( $\mathbf{C}$ from the $Z \rightarrow e e$ mass resolution, $\mathbf{S}$ from the test beam and $\mathbf{N}$ from $W \rightarrow e \nu$ ) and with pions from the test beam for the hadronic section. 
The measurement of the Run II energy resolution of the electromagnetic calorimeter is performed utilizing the three resonances $J / \Psi, \Upsilon$ and $\mathrm{Z}$ that decay to two electrons. The central tracking system provides the momentum measurement from which the energy can be inferred. The energy resolution of the hadronic calorimeter in Run II is measured by randomly selecting collisions (so-called zero-bias events). Most of the time no hard-scatter interaction will occur. The central tracking system is then used to identify single tracks representing isolated charged particles, which are dominantly charged pions. The tracking system provides a momentum measurement which is used to determine the expected energy deposition of the particle in the calorimeter.

\subsubsection{The Muon System}

Muons originating from a $p \bar{p}$ collision penetrate the tracking system and the calorimeter essentially unperturbed because they are minimum ionizing particles $\left(\mathrm{MIPs}^{1}\right)$. The DØ muon detection system is the outermost part of the DØ detector. It is placed around the calorimeter as shown in Figure [Fig 2.21], and it serves to identify and trigger on these muons and to provide a crude measurement of their momenta and charge.

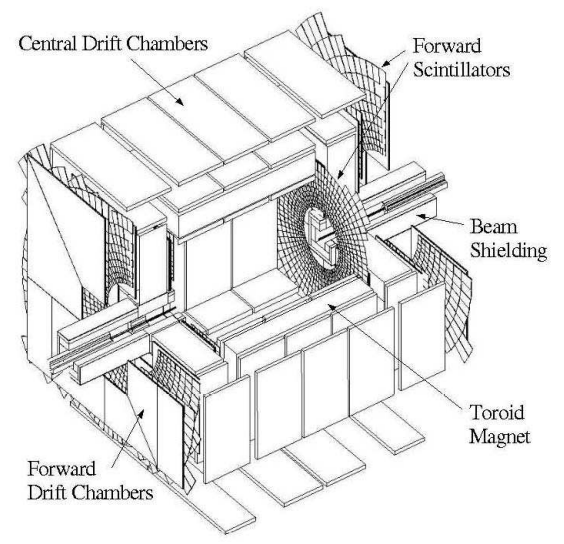

Figure 2.21: Schematic view of the muon system.

It consists of a system of proportional drift tubes (PDTs), mini drift tubes (MDTs) and scintillation counters. The PDTs cover $\left|\eta_{\text {det }}\right|<1.0$, the planes of

\footnotetext{
${ }^{1}$ Cosmic muons and fast electrons are both examples of MIPs as they have low mass and high energy. The energy loss through ionization for a MIP per unit path length is typically a few $\mathrm{MeV} \cdot \mathrm{cm}^{-1}$ material.
} 
MDTs extend the muon detection to $\left|\eta_{\text {det }}\right|=2.0$. The scintillator counters are used for triggering and for cosmic and beam related muon rejection. Toroidal magnets and special shielding complete the muon system. Each sub-system has three layers called A, B, and C. The A layer is innermost and located between the calorimeter and the iron of the toroid magnet. B and $\mathrm{C}$ layers are located outside the iron. In the region directly below the calorimeter, only partial coverage by muon detectors is possible as the support structure for the DØ detector and the readout electronics are located in this region.

The average energy loss of a muon in the calorimeter is $1.6 \mathrm{GeV}$, and about $1.7 \mathrm{GeV}$ in the iron. The momentum measurement is corrected for this energy loss. In the following sections the subsystems of the muon spectrometer are discussed.

\section{Toroid Magnet}

A toroid magnet [20], located between layer A and B, allows a measurement of the muon momentum. It is a square annulus $109 \mathrm{~cm}$ thick, in radial distance of $317.5 \mathrm{~cm}$ from the beam line. In order to give access to the inner portions of the $\mathrm{D} \varnothing$ detector, the central toroid is split in 3 parts. A bottom section gives support for the calorimeter and tracking detectors. The central toroid is completed by two movable c-shaped shells. The two forward toroid magnets are located at $447<|z|<600 \mathrm{~cm}$. The coils carry currents of $1500 \mathrm{~A}$ and result in an internal field of $1.8 T$.

\section{Proportional Drift Tubes}

The PDTs [21] are rectangular volumes, filled with gas covering $\left|\eta_{\text {det }}\right|<1$. The ionization created by a passing charged particle is collected and amplified by a $50 \mu \mathrm{m}$ gold-plated tungsten sense wire which runs through the center of the chamber. Vernier cathode pads are located above and below the wires to provide information on the hit position along the wire. A measurement of the arrival time of the pulse from the sense wire and a calibration of the drift time of the gas allows for calculation of the radial distance from the sense wire. A comparison of arrival times from adjacent wires provides a rough measurement of the position of the ionization along the wire.

PDTs are constructed of extruded aluminum coated with steel foil and filled with a mixture of $84 \%$ Argon, $8 \% \mathrm{CH}_{4}$ and $8 \% \mathrm{CF}_{4}$. The gas flow rate is 500 liters per hour. The drift velocity is approximately $10 \mathrm{~cm} / \mu \mathrm{s}$, for a maximum drift time of about $500 \mathrm{~ns}$. The coordinate resolution of the radial distance to the sense wire is approximately $3 \mathrm{~mm}$. 


\section{Mini Drift Tubes}

The MDTs [22] extend the muon detection to $\left|\eta_{\text {det }}\right|=2$. They have a shorter electron drift time compared to the PDTs $(40-60 \mathrm{~ns}$, depending on the inclination of the tracks), a better coordinate resolution $(\approx 0.7 \mathrm{~mm})$, are radiation hard, and have a high segmentation, thus a low occupancy. They are filled with a mixture of $90 \% \mathrm{CF}_{4}$ and $10 \% \mathrm{CH}_{4}$. Figure [Fig 2.22] shows an expanded view of the drift tubes.

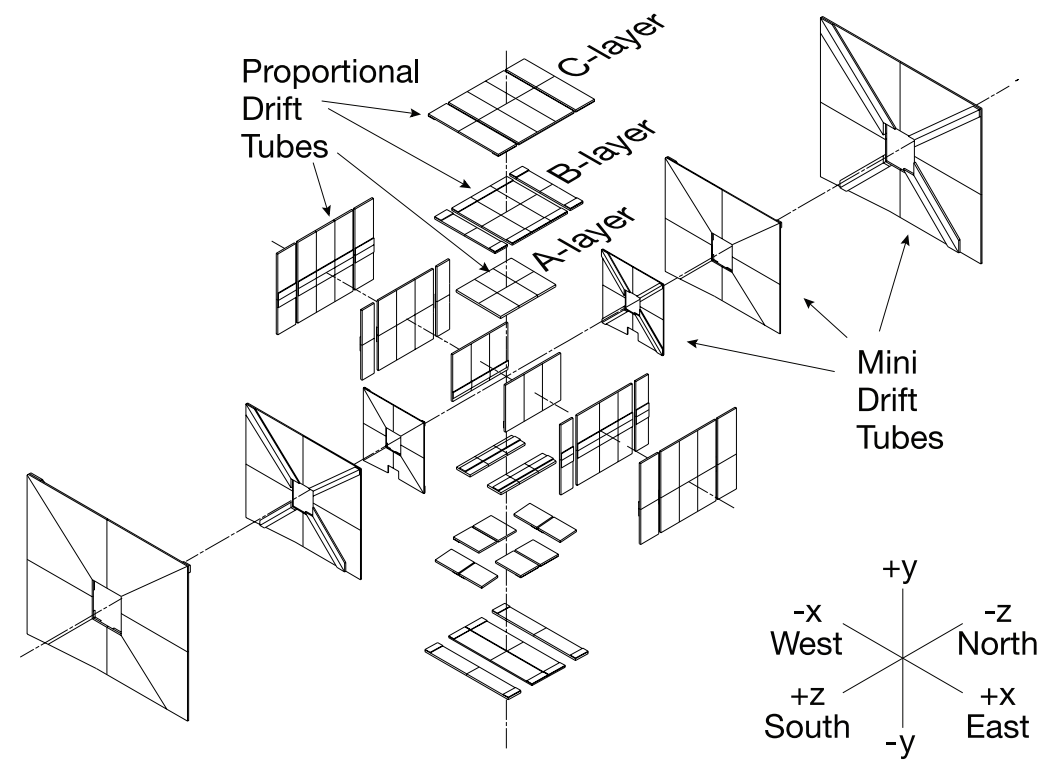

Figure 2.22: View of the three drift chamber layers of the muon system.

\section{Scintillation Counters}

Sheets of scintillating pixels accompany each layer of drift tubes, with the exception of the B layer in the central system. Designed to cover $4.5^{\circ}$ in $\phi$, they provide additional position measurement, and are used for triggering, cosmic ray veto, beam related muon rejection and track reconstruction. Figure [Fig 2.23] shows an expanded view of the scintillation counters [23]. The pixels consist of a slab of scintillator in which light-collecting fibers are set in grooves. A photomultiplier tube collects the light and provides an analog voltage pulse to the digitizing electronics. 


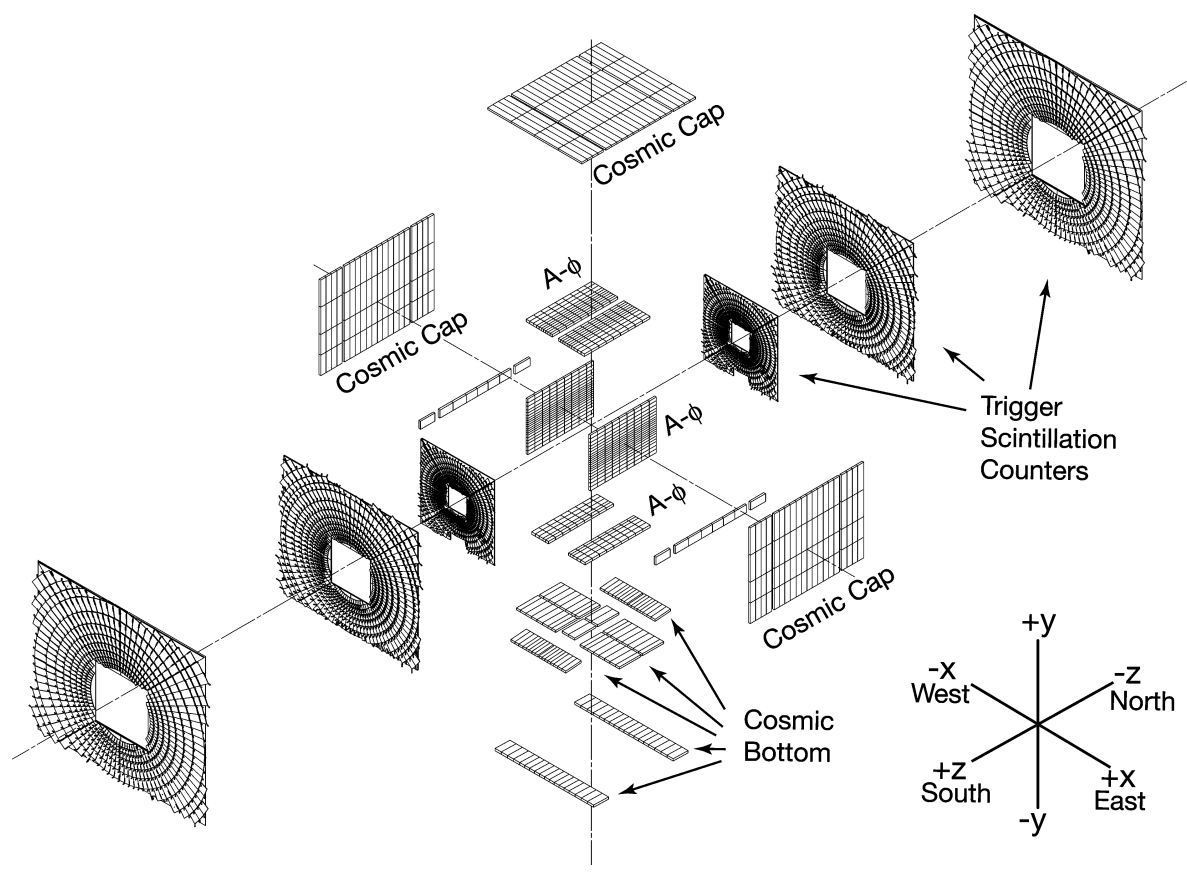

Figure 2.23: View of the three scintillator layers of the muon system.

\section{Shielding}

The shielding consists of layers of iron, polyethylene and lead in a steal structure surrounding the beam pipe and low beta quadrupole magnets. Three different sources contribute to background in the central and forward muon system:

- Scattered proton and antiproton fragments that interact with the end of the calorimeter or with the beam pipe produce background in the central and forward A layer.

- Proton and antiproton fragments, mostly muons from pion decays created by proton and antiproton interactions upstream of the detector, interacting with the low beta quadrupole magnets produce hits in the $\mathrm{B}$ and $\mathrm{C}$ layers of the forward system.

- Beam halo interactions affect both the central and the forward muon system. Iron is used as the hadronic and electromagnetic absorber, polyethylene is a good absorber for neutrons due to its high hydrogen content, and lead is used to absorb gamma rays. 


\section{Performance Muon System}

The performance of the muon system combined with the central tracker is shown in Figure [Fig 2.24], where the di-muon invariant mass is shown at the $\omega, \phi, J / \Psi, \Psi^{\prime}, \Upsilon$ and at the $\mathrm{Z}$ mass.
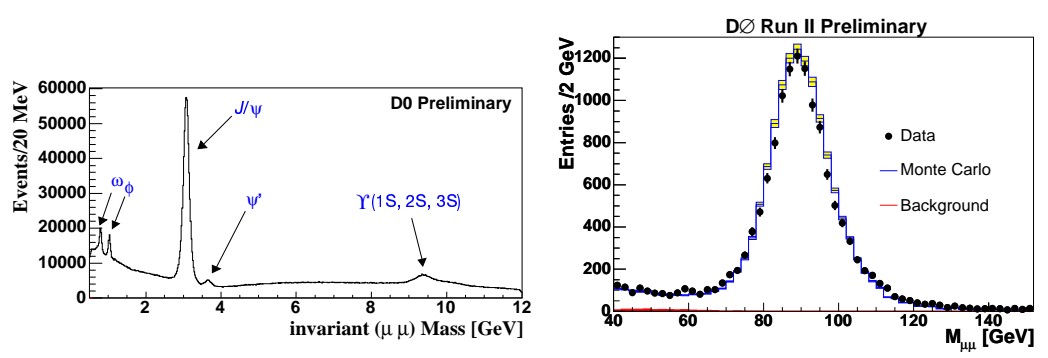

Figure 2.24: $\mu^{+} \mu^{-}$invariant mass distributions of various meson resonances: $\omega, \phi, J / \Psi, \Psi^{\prime}, \Upsilon$ and the $\mathbf{Z}$ mass.

The momentum resolution of the muon system has been studied using reconstructed muons with an associated central track. The muon momentum resolution, $\sigma\left(p_{T}\right) / p_{T}$, as measured by the muon system, varies between 0.1 for low-momentum muons and 0.5 for muons with $p_{T}>50 \mathrm{GeV}$ [24]. The overall muon momentum resolution, including information from the silicon vertex detector and the central fiber tracker, is defined by the central tracking system for muons with momentum up to approximately $100 \mathrm{GeV}$. The muon system measurement improves the resolution only for higher momentum muons [8].

\subsubsection{Forward Proton Detector (FPD)}

The Forward Proton Detector (FPD) [25] is a series of momentum spectrometers that make use of accelerator magnets in conjunction with position detectors along the beam line in order to determine the kinematic variables $t$ and $\sigma$ of the scattered $\mathrm{p}$ and $\bar{p}$, where $|t|$ is the four-momentum transfer of the scattered proton or antiproton, and $\xi=1-x_{p}$, where $x_{p}$ is the fractional longitudinal momentum of the scattered particle with respect to the incoming proton. The FPD covers the region $0 \leq t \leq 4.5 \mathrm{GeV}^{2}$.

The position detectors must operate a few millimeters from the beam and have to be moved away during the injection of protons in the accelerator. Special devices, called Roman pots [26], are designed to house the position detectors allowing for remotely controlled movement with an accuracy in the order of tens of microns. 
The Roman pots are housed in stainless steel chambers called castles. The FPD, shown in Figure [Fig 2.25], consists of 18 Roman pots arranged in six castles, where the detectors placed in each castle can approach the beam from up, down, inside and outside directions with respect to the Tevatron ring. Four castles are located downstream of the low beta quadrupole magnets on each side of the colliding point: two on the proton side (P1 and P2) and two on the antiproton side (A1 and A2). The FPD is not used in the present analysis.

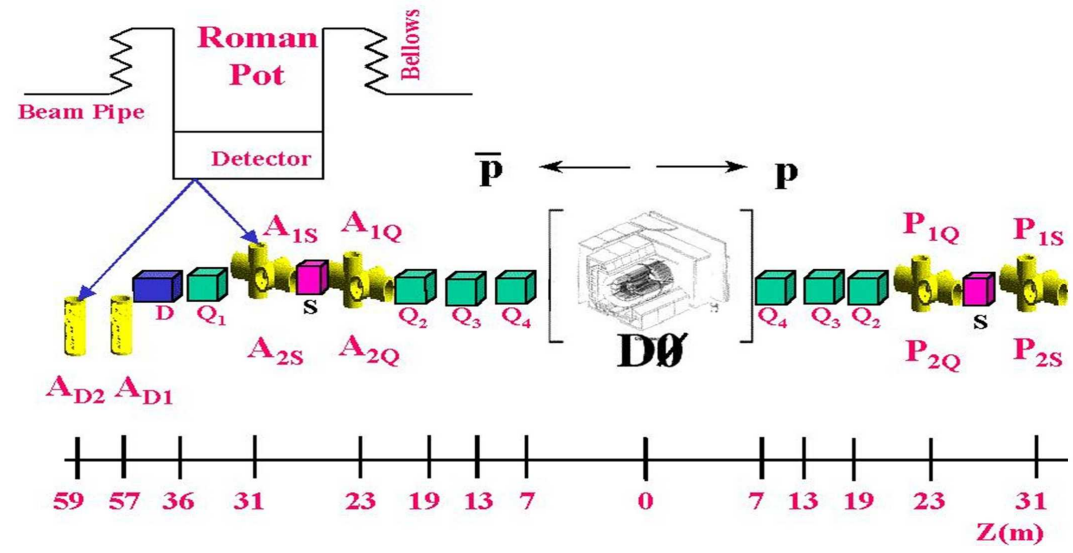

Figure 2.25: Schematic view of The Forward Proton Detector (FPD). Quadrupole Roman pot detectors are named $\mathbf{P}$ or $\mathbf{A}$ when placed on the $\mathbf{p}$ or $\bar{p}$ side, respectively. Dipole pots, located on the $\bar{p}$ side, are named $\mathrm{D}$.

\subsubsection{Trigger System and Data Acquisition System}

At the Tevatron, $p \bar{p}$ collisions occur at a rate of $2.5 \mathrm{M} \mathrm{Hz}$ and it is not possible to record and store events at this rate. Since most of the interactions are $p \bar{p}$ inelastic collisions and are not of interest, a triggering system has been developed to select the interesting physics events to be recorded. In addition to the luminosity monitor, there are three separate levels in the trigger system with each succeeding level examining fewer events but in greater detail and with more complexity. These levels are named Level 1, Level 2 and Level 3 and are shown as part of an overview of the trigger and data acquisition system in Figure [Fig 2.26]. The luminosity monitor provides the first stage in rate reduction by rejecting events in which no hard $p \bar{p}$ interaction has taken place, thus reducing the L1 rate. 


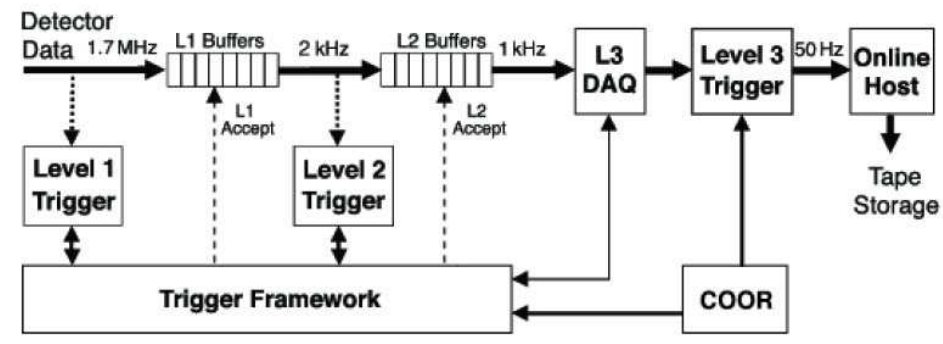

Figure 2.26: Overview of the $\mathrm{D} \emptyset$ trigger and data acquisition systems

\section{Level 1 Trigger(L1)}

The first trigger stage (Level 1 or L1) is a hardware trigger that consist of a framework built of field programmable gate arrays (FPGAs), which take inputs consisting of simple objects provided by the individual subdetectors (luminosity monitor, calorimeter and muon system). It has a pipeline, which allows to make a decision within $4.2 \mu \mathrm{s}$, resulting in a trigger accept rate of about $2 \mathrm{kHz}$.

The luminosity system provides an indication that a collision occurred with a position on the $z$ axis which would place it within the D $\varnothing$ detector. The calorimeter employs a special path which performs a very quick summation of electromagnetic and hadronic towers at a resolution of $\Delta \eta \times \Delta \phi=0.2 \times 0.2$, excluding the coarse hadronic section due to a higher noise rate. The trigger requires that the energy in these towers be above a certain threshold. Based on signal to noise considerations, only the trigger towers for $\left|\eta_{\text {det }}\right|<3.2$ are used. Additional trigger terms are possible for global quantities such as the total sum of all tower energies, $\sum E_{T}$, and the missing transverse energy. The muon trigger requires a coincidence between the scintillators in the $\mathrm{A}$ and $\mathrm{B}$ or $\mathrm{C}$ layers.

\section{Level 2 Trigger (L2)}

In the second stage (Level 2 or L2), hardware engines associated with specific subdetectors process information that is then used by a global processor to determine correlations between different detectors, e.g. matching tracks and leptons. Level 2 has an accept rate of $1 \mathrm{kHz}$ at a maximum dead-time of $5 \%$, and a maximum latency $^{2}$ of $100 \mu s$. The Level 2 trigger passes events to the Level 3 system.

Figure [Fig 2.27] shows the design of the DØ Level 1 and Level 2 trigger system.

\footnotetext{
${ }^{2}$ latency: time taken to make a decision
} 


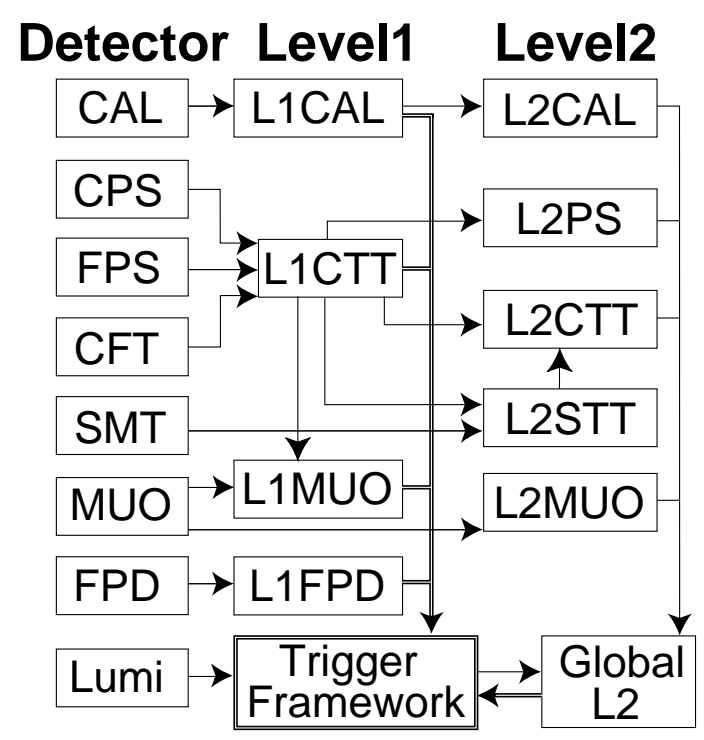

Figure 2.27: Schematic view of subdetectors with L1 and L2 trigger elements. Horizontal arrows indicate the direction of dataflow.

\section{The Level 3 Trigger (L3)}

The Level 3 trigger provides additional rejection of events to enrich the physics samples and to reduce the rate of events to be stored on tape to $50 \mathrm{~Hz}$ for offline analysis. The accept rate is limited by the capabilities of and cost of data storage and offline computing. L3 is entirely software-based, reads out information from the entire detector and is run on a dedicated PC farm. Software tools build on the basic physics objects of L2 and decisions are using these objects and the relationships between them such as invariant mass. The filtering software can be changed as physics objectives or the operating parameters of the experiment change.

The list of filters and their criteria is known as a trigger list and is assigned a version number. 


\section{Chapter 3}

\section{Object Identification}

This chapter briefly describes how different particles interact with the DØ detector. It also explains how they are measured, calibrated, and the terminology associated with them.

\subsection{Charged Tracks}

The central tracking system accounts for a large fraction of the event data. Scanning through all of its channels for signals of charged particles is one of the most time consuming tasks of the reconstruction chain. Charged particles, curving through the magnetic field, leave traces in the central tracking system from which tracks are reconstructed.

The reconstruction is divided in two parts: hit clustering, that groups individual channels that are likely to represent the passage of an individual particle, and track finding, which finds groups of clusters located along a physical path. Particles passing through the SMT will deposit charge in a number of strips. A particle traversing the CFT will illuminate a number of fibers. In both cases, a cluster is defined as a group of adjacent strips above a noise threshold. The track finding is subdivided into two algorithms: pattern recognition and track fitting. The pattern recognition creates sets of clusters which lie along physical paths. The track fitting uses sophisticated algorithms (Kalman filter [32]) to fit a candidate charged particle track to a physical path, using a $\chi^{2}$ test.

\subsection{Primary Vertex}

The Primary Vertex (PV) is defined as the location of a $p \bar{p}$ collision. The reconstruction of Primary Vertices consists of two major steps [33]: 
1. The algorithm locates the position of the beam spot center. At this step, all tracks with distance of closest approach (dca) significance $S_{(0,0)}<100$, calculated with respect to $(x, y)=(0,0)$ in the transverse plane, are fitted to the PV. The result of the fit is the list of possible primary vertices.

2. at the second pass, the track dca significance is calculated with respect to the position of these first-pass vertices. Only tracks with at least two SMT hits, surviving a tight dca significance cut, are fitted to the final primary vertices.

The final step is the selection of the hard scatter vertex from the list of reconstructed vertices. The method used is described in detail in [34]. It is based on the fact that tracks from minimum bias interactions have smaller transverse momenta than tracks from hard scatter interactions. The $\log _{10} p T$ distribution of tracks from minimum bias processes is used to define a probability for a track to come from a minimum bias vertex. For each vertex, the product of the probabilities of each track divided by the total number of tracks is calculated, thus forming the probability for a vertex to originate from a minimum bias interaction. The $\mathrm{PV}$ with the lowest minimum bias probability is chosen as the hard scatter PV.

The eficiency of the PV reconstruction is about $100 \%$ in the central $|z|$ region and drops quickly outside the SMT fiducial volume $(|z|<36 \mathrm{~cm}$ for the barrel) due to the requirement of two SMT hits per track forming the PV. The resolutions (convoluted with the beam spot size) of $x, y$ and $z$ coordinates of the reconstructed PV depend on the number of tracks, $N_{\text {trks } P V}$, fitted into the PV, and are completely dominated by the beam spot starting from $N_{\text {trks } P V}>15$. The resolution is about $35 \mu \mathrm{m}$ in the transverse plane.

\subsection{Muons}

Muons are reconstructed using information from two independent detector systems: the muon detector and the central tracker. A local track in the muon system is the basis of the muon identification. Muons are required to have hits in all three layers of the muon system (both inside and outside the toroid).

The following standard Muon ID requirements are used:

- Muon candidates are required to be of medium quality ${ }^{1}$ according to the Muon ID criteria [48].

\footnotetext{
${ }^{1}$ See appendix A
} 
- A loose cut against cosmics is applied, based on timing information from scintillator hits associated with the muon that requires A-layer and BC-layer scintillator times $\left|t_{A}\right|<10 \mathrm{~ns}$ and $\left|t_{B C}\right|<10 \mathrm{~ns}$

- the muon is required to be at $|\eta|<2.0$.

In addition a central track match is required. This means that the muon tracks are extended to the point of closest approach (PCA) to the beam and their parameters are compared with those of tracks in the central tracker at the PCA. A global fit is performed with all central tracks within 1 radian in azimuthal and polar angle of a muon track at PCA. The central track with the highest $\chi^{2}$-probability is considered to belong to the muon candidate. The measurement of the muon track parameters are taken from the central tracking system. The following additional quality requirements are applied to the central track:

- $\chi_{\text {track }}^{2} / d o f<4$ for the central track fit, to remove bad track fits.

- A distance $|\Delta z(\mu, P V)|<1 \mathrm{~cm}$ between the track and the primary vertex, to further reduce background from cosmics and badly reconstructed tracks.

- $d c a$ signicance less than 3 standard deviations away from zero, $d c a / \sigma_{(d c a)}<3$, in order to reject muons from semi-leptonic heavy flavor decays.

A correction is applied to the momentum of muons matched to CFT-only tracks, i.e. those tracks where no hits are found in the SMT subdetector. The procedure considers the primary vertex as a constraint for the fit [48]. The track is refitted such that the $d c a$ in $\mathrm{x}$ and $\mathrm{y}$ remains 0 [44]. The correction factor is given by:

$$
S F_{C F T-o n l y}=1-d c a / q o p t \cdot(E R R(r, q o p t) / E R R(r, r)),
$$

where $d c a$ is the muon $r-\phi$ distance of closest approach to the primary vertex, qopt is the muon charge divided by the muon $p_{T}, E R R(r, q o p t)$ and $E R R(r, r)$ represent the respective error matrix entries where $r$ is the $r-\phi$ impact parameter relative to $(x, y, z)=(0,0,0))$.

The main background for identification of muons comes from semileptonic decays in heavy quark jets. Compared to muons originating from the leptonic decay of a $\mathrm{W}$ boson, those muons tend to be non-isolated and have a lower transverse momentum. Muon isolation is used to distinguish muons from these two different sources. A loose isolation criteria is defined by demanding that a muon is separated from a jet, $\Delta R(\mu$, jet $)>0.5$.

The muon isolation criteria are [35]: 
- Halo(0.1,0.4) $<2.5 \mathrm{GeV}$, where $\operatorname{Halo}(0.1,0.4)$ is the sum of the ET of calorimeter clusters in a hollow cone between $\Delta R=0.1$ and $\Delta R=0.4$ away from the muon. In forming this sum, cells in the electromagnetic and fine hadronic calorimeters are considered. Due to an enhanced noise level, the coarse hadronic calorimeter is excluded from the sum.

- $\operatorname{TrkCone}(0.5)<2.5 \mathrm{GeV}$, where TrkCone $(0.5)$ is the sum of the $p_{T}$ of all tracks within a cone of radius $\Delta R=0.5$ surrounding the muon. The track matched to the muon is excluded from this sum.

\subsection{Electrons}

Electrons and positrons lose energy primarily through ionisation and bremsstrahlung [36]. Bremsstrahlung is the radiation emitted by a charged particle when accelerated transversely when passing through the field of atomic nuclei. This results in a shower of electron-positron pairs and photons. The photons themselves produce electron-positron pairs which further shower in the fields of the nuclei. For electrons above the critical energy of $E \approx 610 \mathrm{MeV} /(1.2+Z$ ) (where $\mathrm{Z}$ is the atomic number of the material), bremsstrahlung is the dominant process. Similarly, a shower is produced if the primary particle is a photon. A radiation length, $\chi_{0}$ can be defined, over which the electron's energy will be reduced by a factor of $1 / e$ [36]:

$$
\chi_{0}=\frac{716 \mathrm{~g} \cdot \mathrm{cm}^{-2} A}{Z(Z+1) \ln (287 / \sqrt{Z})}
$$

The reconstruction of electrons and other EM objects starts in the calorimeter with clusters of energy deposits in cells in the EM layers $\left(E_{E M}\right)$, and the first hadronic layer $\left(E_{\text {had }}\right)$. A cluster is defined to be a group of $3 \times 3$ towers with energy greater than $500 \mathrm{MeV}$ [37]. The primary method to reconstruct electrons proceeds with a simple cone algorithm, with $\mathrm{R}=0.2$, which is then applied to the seed-towers with $E_{T}>1.5 \mathrm{GeV}$. At this point, photons and electrons may be differentiated since electrons are expected to interact with the tracking system, and photons are not. An electron track, which must have $p_{T}>1.5 \mathrm{GeV}$, should point in the same direction as the electron's EM shower, and have a momentum that matches the energy of the shower. This is measured using a $\chi^{2}$ match quantity [38]:

$$
\chi_{\text {spatial }}^{2}=\left(\frac{\delta \phi}{\sigma_{\phi}}\right)^{2}+\left(\frac{\delta z}{\sigma_{z}}\right)^{2}+\left(\frac{E_{T} / p_{T}-1}{\sigma_{E_{T} / p_{T}}}\right)^{2}
$$

Electrons require a $\chi^{2}$ probability of greater than $10^{-2}$. The track now associated with the electron provides the $\theta$ and $\phi$ coordinates, and its total energy is 
taken from the calorimeter measurements; combining them gives the momentum. Without a track match, these coordinates are reconstructed from the primary vertex and the object's position within the third EM layer. Several additional variables are useful in characterising electrons and photons with high efficiency:

- Isolation Fraction $\left(f_{i s o}\right)$ : Electrons and photons (and the showers they produce) are usually isolated from other particles in the calorimeter, unlike hadrons which are usually in jets of particles. The measure of this isolation, the isolation fraction, dependent on the total energy within the cone from all layers used, $E_{t o t}$, is given by:

$$
f_{\text {iso }}=\frac{E_{t o t}(R<0.4)-E_{E M}(R<0.4)}{E_{\text {tot }}(R<0.2)}
$$

where $\mathrm{R}$ defines the size of the cone.

- EM Fraction $\left(f_{E M}\right)$ : This uses the measurement of the energy deposited in the first hadronic layer, with the energy deposited in the EM layers to calculate the fraction of energy deposited in the EM calorimeter:

$$
f_{E M}=E_{E M} / E_{t o t} .
$$

- Coarse Hadronic Fraction $\left(f_{C H}\right)$ : In a similar way to $f_{E M}$, the coarse hadronic fraction is the fraction of energy deposited in the coarse hadronic layers.

- Hot Fraction: This is the ratio of the transverse energies of the calorimeter tower with the highest energy, to the tower with the second highest energy. This cut, along with the following two variables, n90 and f90, are used to eliminate hot cells in the calorimeter that have been misidentified as EM objects.

- n90: This is the total number of towers that make up 90\% of the EM shower energy.

- f90: Similar to n90, this is the fraction of the number of cells comprising $90 \%$ of the EM object energy.

- HMatrix ( $H M x 8)$ : This measures how so similar the shower is to an electron shower. There are eight correlated observables used in this shower shape analysis that include EM energy fractions, the total EM energy, vertex z-position and transverse shower width in $\phi$ and $\mathrm{z}$. The $8 \times 8$ covariance matrix is a measure of the shower similarity. The HMatrix is the inverse of this covariance matrix. It is calculated for each tower in $\eta$ [39] 
There are several sources of background which may be mistakenly identified as electrons [39]

- $\pi_{0}$ showers which overlap with a track from a charged particle.

- Photons which convert to $e^{+} e^{-}$pairs.

- $\pi^{ \pm}$which undergo charge exchange in the detector material.

- Fluctuations of hadronic shower shapes.

\subsection{Jets}

In $p \bar{p}$ collisions, final state partons are produced which hadronise into collimated streams of particles, labelled jets. These are composed predominantly of photons, pions and kaons and they are detected when the particles deposit energy in the calorimeter cells. Jets are then reconstructed from these energy deposits using the DØRun II jet cone algorithm [40], [41]. To relate the measured jet energy back to the energy of the final state partons, a jet energy scale is derived to correct for the distortions to the measured energy by various detector and physics effects.

Hadrons interact with the nuclei of the detector. A length scale, the interaction length [36], similar to the radiation length of electrons, is given approximately by:

$$
\lambda_{I} \approx 35 A^{1 / 2} \mathrm{~g} \cdot \mathrm{cm}^{-2}
$$

Hadrons produce showers of hadronic particles, mostly pions and nucleons, which in turn interact with the nuclei of the detector producing a cascade. These showers are measured as jets within the calorimeter; clustering algorithms group the cells with energy deposits from the EM and hadronic layers. Cones reconstructed with the jet cone algotithm have their origins at an interaction point and extend outwards containing the jet particles. They have a fixed $\Delta R$ in $\eta-\phi$ space. The cone jet corresponds to a "stable" orientation of the cone around energy clusters in the calorimeter. A seed-based algorithm is applied which uses seeds and midpoints of seeds as starting points for finding these stable cones. The seeds are preclusters of calorimeter towers, instead of single (out of 5000) towers, which reduce the time taken to find all stable cones. The Run II cone algorithm is divided into three stages: clustering, addition of midpoints, and merging-splitting. The clustering stage is based on the Simple Cone Algorithm and it forms the preclusters of towers which are used to form protojets. Following this, protojets are also searched for around the midpoints of any two already existing protojets if they are more than $\Delta R_{\text {cone }}$ (usually $\mathrm{R}=0.5$ ) apart. Protojets often share items (clusters) 
of energy, cones are merged or split according to how the clusters are shared. This is to avoid double counting of energy. If a protojet shares one or more items with another protojet and these represent $50 \%$ or more of the energy of the other jet, then the shared clusters are assigned to that other jet, and the first jet is removed. If the fraction of shared energy is smaller than $50 \%$ of either jet, then the two jets are split and the items are assigned to the nearest jets.

\subsection{Missing $E_{T}\left(E_{T}\right)$}

The presence of a neutrino in the final state can be detected only from the imbalance of the energy in the transverse plane. The missing $E_{T}\left(E_{T}\right)$ is reconstructed from the vector sum of the transverse energies of all cells surviving the T42 algorithm $^{2}$, except for those in the coarse hadronic layer which are treated separately due to their high level of noise. The only cells of the coarse hadronic calorimeter which are used in the $E_{T}$ sum are those clustered within good jets. The vector opposite to this total visible momentum vector is denoted the missing energy vector and its modulus is the raw missing transverse energy $\left(\mathbb{E}_{T} \cdot\right.$ raw $)$. So, $\mathbb{E}_{T}$ is the negative magnitude of the vector sum of the calorimeter tower transverse energies and is defined as:

$$
E_{T}=-\left(\sum_{i}\left(E_{x i}\right), \sum_{i}\left(E_{y i}\right)\right)
$$

where $E_{x i}$ and $E_{y i}$ are defined the x and y components of the tower energies within $|\eta|<4.5$. Before the $E_{T}$ can be used in an analysis, it must be adjusted to account for the corrections that are applied to the other physics objects in the event [42]: noisy cells in the coarse hadron calorimeter, the EM scale, the jet energy scale, and for muons detected only in the muon system. Where jets are concerned, the scalar hadronic transverse energy, $H_{T}$ is sometimes used. This is the scalar sum of the $p_{T}$ of the good jets in an event. Also used is the missing $H_{T}\left(H_{T}\right)$, which is the negative magnitude of the vector sum of the jet transverse energies.

\footnotetext{
${ }^{2}$ See appendix D
} 


\section{Chapter 4}

\section{Measurement of the Production Cross Section of Diffractive $Z^{0} / \gamma^{*} \rightarrow \mu^{+} \mu^{-}$with Gap}

This chapter describes a measurement of the diffractive $\left(Z^{0} / \gamma^{*} \rightarrow \mu^{+} \mu^{-}\right)$production cross section with gap, multiplied by the muon branching ratio, also, we measure the gap fraction of $\mathrm{Z}$ bosons produced diffractively to all $\mathrm{Z}$ bosons and we measure $d \sigma / d \xi, d \sigma / d y$ distributions in $p \bar{p}$ collisions at a center of mass energy of $1.96 \mathrm{TeV}$.

\subsection{Data Sample}

The data sample used for this analysis corresponds to data recorded by the D $\varnothing$ experiment from 2003 to 2006, starting from run number 178069 (June 2003) up to run number 214999 (February 2006). The integrated luminosity for this dataset is $\int \mathcal{L} d t=820 \mathrm{pb}^{-1}$. We use skim data with two muons with high transverse momentum $\left(p_{T}>15 \mathrm{GeV}\right)$. The number of events stored are about 9.26 million events. Runs and luminosity blocks in which the SMT,CFT, calorimeter or muon detector system were not working properly or in which the integrated luminosity could not be calculated have been excluded.

\subsection{Selection Criteria}

The first stage in the analysis is to isolate the $Z \rightarrow \mu^{+} \mu^{-}$signal. To do this, we use standard cuts in order to obtain the $\mathrm{Z}$ boson candidates, which are explained below. The $p_{T}$ of the muons is measured with the central tracking system because 
this detector has a better resolution than the muon detector system. In case that the muon track does not have hits in the SMT detector the $p_{T}$ is corrected by constraining the track to the beam spot position. We have acceptance cuts to exclude the parts of the muon detector where there is not coverage: the regions nearest the beampipe, $\left|x_{A}\right|<110 \mathrm{~cm}$ and $\left|y_{A}\right|<110 \mathrm{~cm}$, where $x_{A}$ and $y_{A}$ are the $x$ and $y$ positions measured in the muon chamber A-layer, and the region of the bottom gap, $4.25<\phi<5.15$ for $\left|\eta_{A}\right|<1.25$, where $\eta_{A}$ is also measured in the A-layer.

The rest of the selection criteria are [44]:

1. Two Loose ${ }^{1}$ quality muons matched to two central detector tracks.

2. Both muons must be within the geometrical acceptance of the muon detector $(|\eta|<2.0)$ [Fig 4.1].

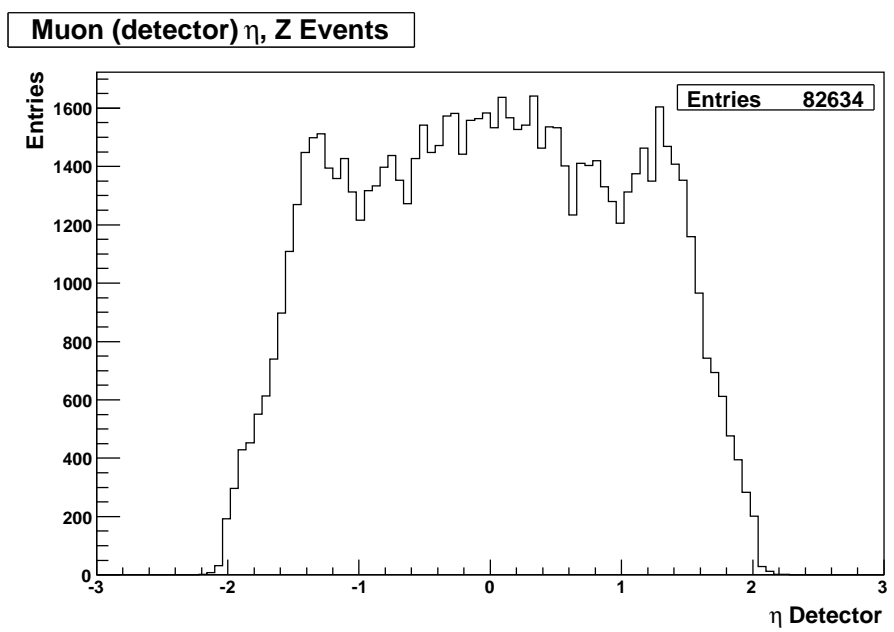

Figure 4.1: Muon $\eta_{\text {det }}$ Distribution

3. $p_{T}>20 \mathrm{GeV}$ for both muons [Fig 4.2].

4. Di-muon invariant mass $M_{\mu \mu}>40 \mathrm{GeV}$ [Fig 4.3].

5. The pseudo-acolinearity, $\Delta \alpha_{\mu \mu}=\left|\Delta \phi_{\mu \mu}+\Delta \theta_{\mu \mu}-2 \pi\right|$, between the two muons is required to be $>0.05 . \Delta \phi_{\mu \mu}$ and $\Delta \theta_{\mu \mu}$ are the differences in the muon $\phi$ and $\theta$ measurements [Fig 4.4].

\footnotetext{
${ }^{1}$ See appendix $\mathrm{A}$ for muon quality definitions
} 


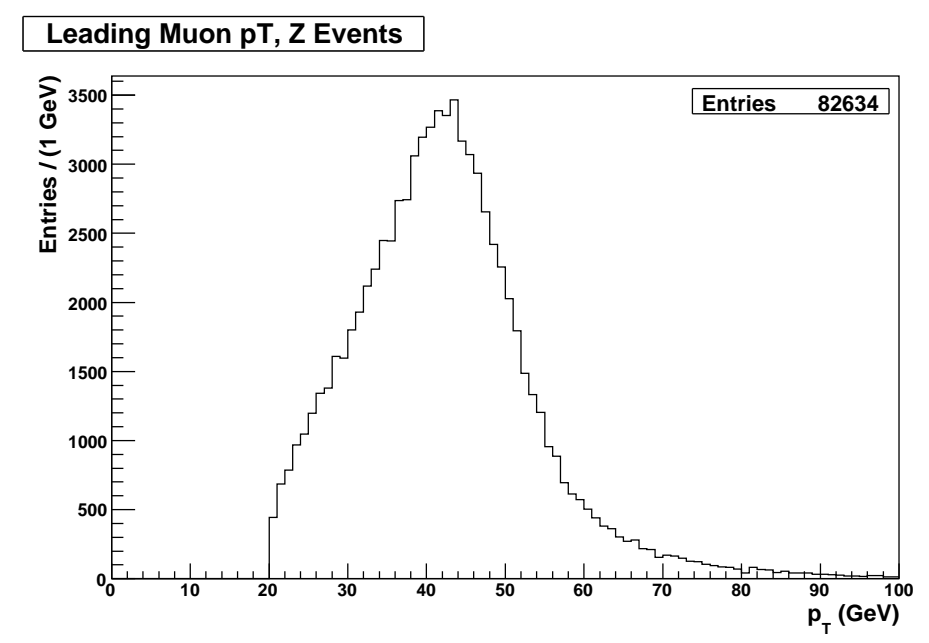

Figure 4.2: Muon $p_{T}$ Distribution

6. The two muons are required to have opposite charge.

7. For muon tracks containing SMT hits the distance of closest approach of the muon vertex to the beam spot (dca) should satisfy $d c a<0.02 \mathrm{~cm}$ and for muon tracks with no SMT hits $d c a<0.2 \mathrm{~cm}$ [Fig 4.5].

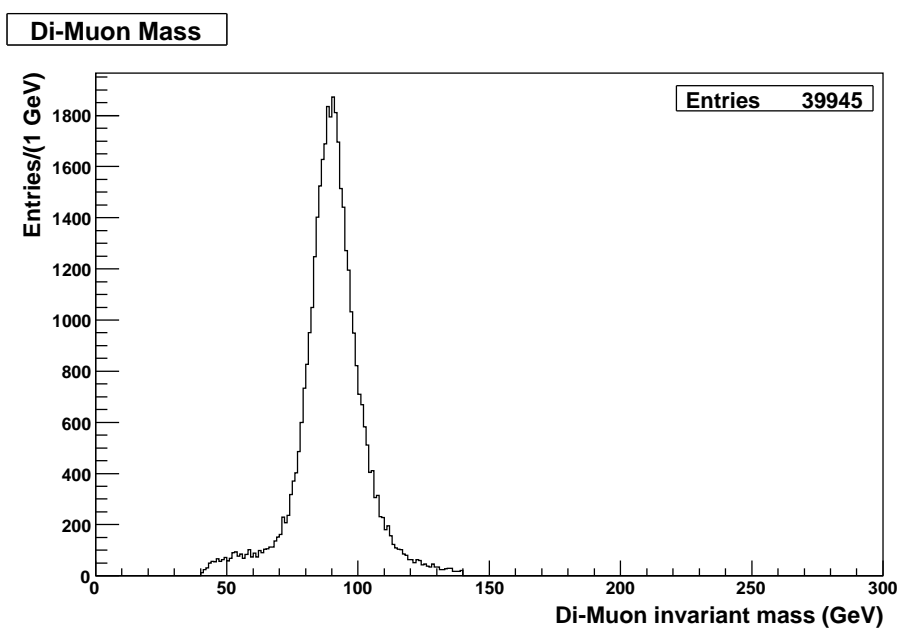

Figure 4.3: Di-Muon Invariant Mass 


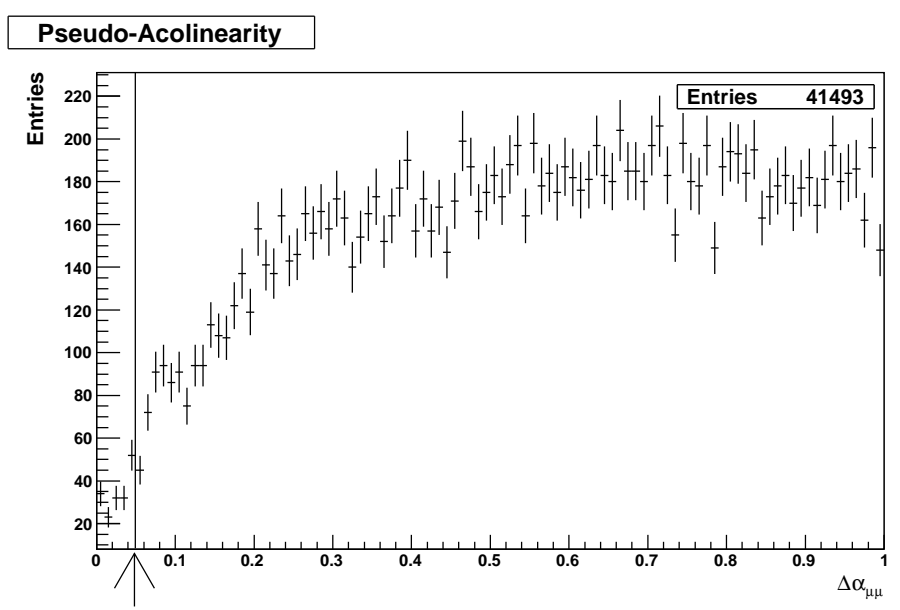

Figure 4.4: Pseudo-acolinearity Plot, the arrow indicates the cut applied in this variable

8. At least one muon should pass both of the following isolation criteria, or both muons should pass at least one of them:

(a) $\sum_{i=1}^{n, \text { tracks }} p_{T}^{i}<3.5 \mathrm{GeV}$, where $\sum_{i=1}^{n, \text { tracks }} p_{T}^{i}$ is the sum of the $p_{T}$ of tracks
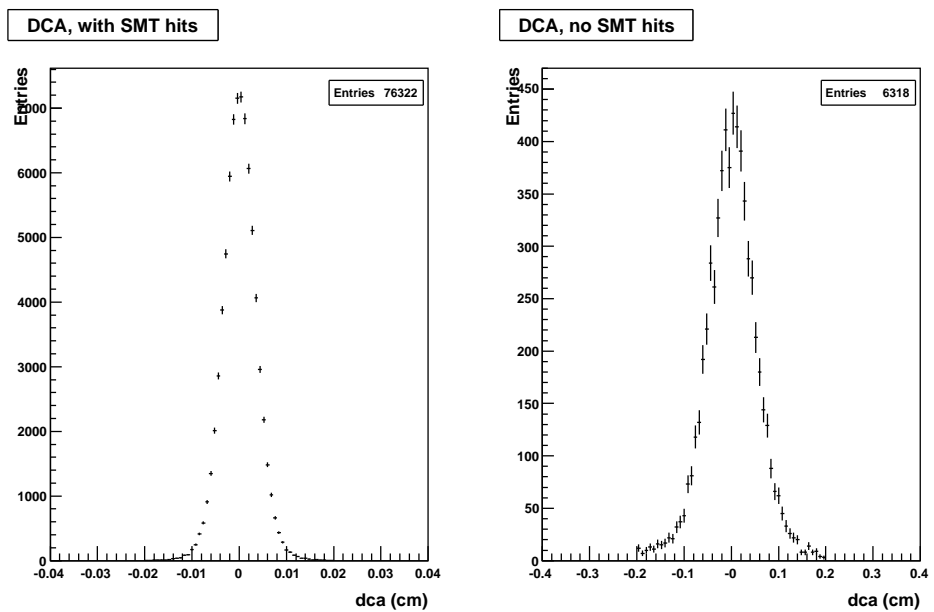

Figure 4.5: Events that pass dca cuts in CFT and SMT 
contained within a cone around the muon track direction with opening angle $R<0.5$, where $R=\sqrt{(\Delta \eta)^{2}+(\Delta \phi)^{2}}$. The tracks are also required to be $<2 \mathrm{~cm}$ in $z$ axis from muon track.

(b) $\sum_{i=1}^{n, \text { cells }} E_{T}^{i}<2.5 \mathrm{GeV}$ where $\sum_{i=1}^{n, \text { cells }} E_{T}^{i}$ is the sum of the transverse energies of calorimeter cells for $0.1<R<0.4$ around the direction of the muon. Cells in the electromagnetic and fine hadronic calorimeters are used, but not those in the coarse hadronic calorimeter due to poor energy resolution in its cells. Event that pass isolation requirements are shown in the figure [Fig 4.6].
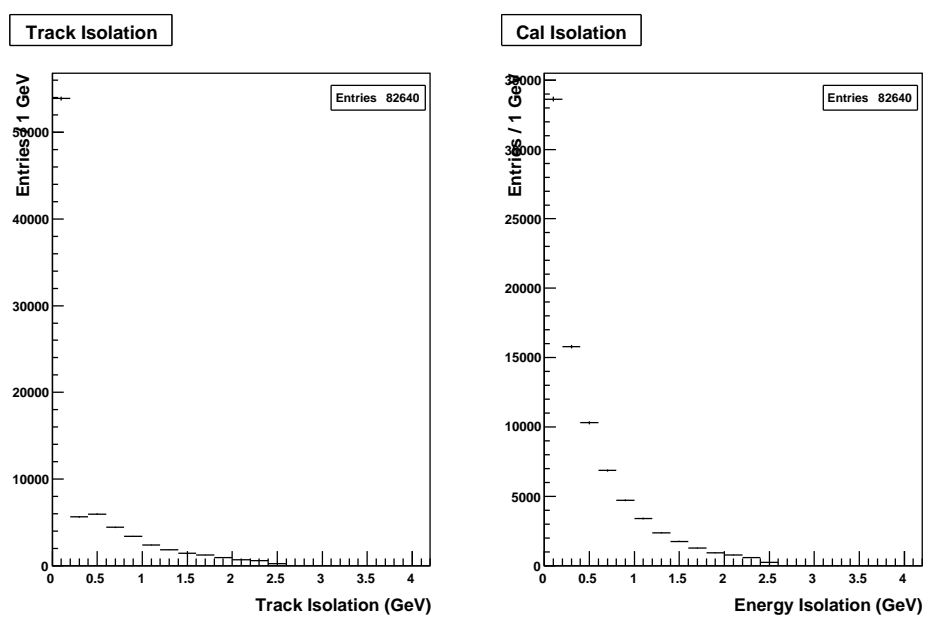

Figure 4.6: Events that pass isolation cuts

9. Single Muon Triggers that were the most fired in each trigger version were used in this analysis. Table 4.1 shows the Integrated Luminosity by trigger version.

\begin{tabular}{|c||c||c|}
\hline Version & Trigger Name & Integrated Luminosity $\left(p b^{-1}\right)$ \\
\hline V12 & MUW_W_L2M3_TRK10 & 224.14 \\
\hline V13 & MUH1_TK12_TLM12 & 330.48 \\
\hline V14 & MUH1_TK12_TLM12 & 265.17 \\
\hline
\end{tabular}

Table 4.1: Integrated Luminosity by trigger version 
The isolation cuts are used to reduce the background from $b \bar{b}$ events in which muons are produced inside jets.

Another background source corresponds to cosmic rays, the pseudoacolinearity requirement reduces this background. When a cosmic ray muon passes through the interaction region it can appear as two tracks from the beam pipe. The tracks are exactly back-to-back, with $\Delta \phi=\Delta \theta=\pi$ radians. Events with two muons into $\Delta \alpha_{\mu \mu}=\left|\Delta \phi_{\mu \mu}+\Delta \theta_{\mu \mu}-2 \pi\right|<0.05$ are considered cosmic background [Fig 4.7]. Aditionally to this cut, we take a look at the distance of closest approach $(d c a)$ that corresponds to the distance between the muon tracks and the beam spot [Fig 4.8]. Tracks reconstructed coming from cosmic muons will have the origin point far to the beam spot. Muon tracks with $d c a>0.2 \mathrm{~cm}$ are excluded.

Cosmic:
Acolinearity $\sim 0$

Z-->mumu: Acolinearity $>0$ (i.e. $Z$ is boosted)
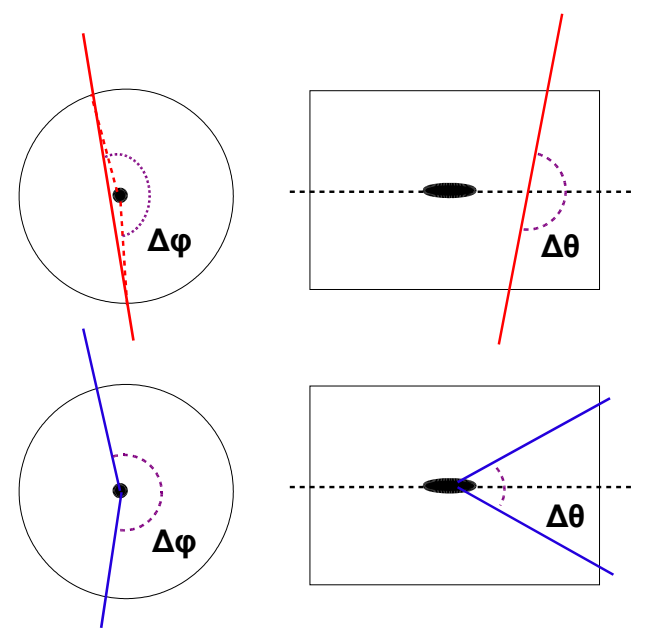

Figure 4.7: Acolinearity Cut

Additional sources of background come from $Z / \gamma^{*} \rightarrow \tau^{+} \tau^{-}$events, $W \rightarrow \mu \nu$ events containing an additional muon inside a jet and di-boson (WW,WZ and ZZ) events.

The total number of candidate events that pass the selection criteria is 39955 . Table 4.2 shows the number of events after each selection cuts. Figure [Fig 4.9] shows the $p_{T}$ and the Rapidity distribution for these di-muon candidates. 
distance of closest approach (dca)

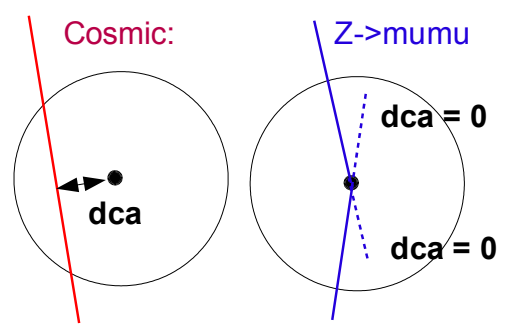

Figure 4.8: Distance Closest Approach Cut

\begin{tabular}{|c||c|}
\hline Selection Cuts & Number of events \\
\hline \hline Skim Data - Two muons with $p_{T}>15 \mathrm{GeV}$ & 86148 \\
\hline Data Quality & 70997 \\
\hline Trigger Selection and Muon quality & 43067 \\
\hline Z kinematical cuts & 39955 \\
\hline
\end{tabular}

Table 4.2: Number of events - Z selection criteria
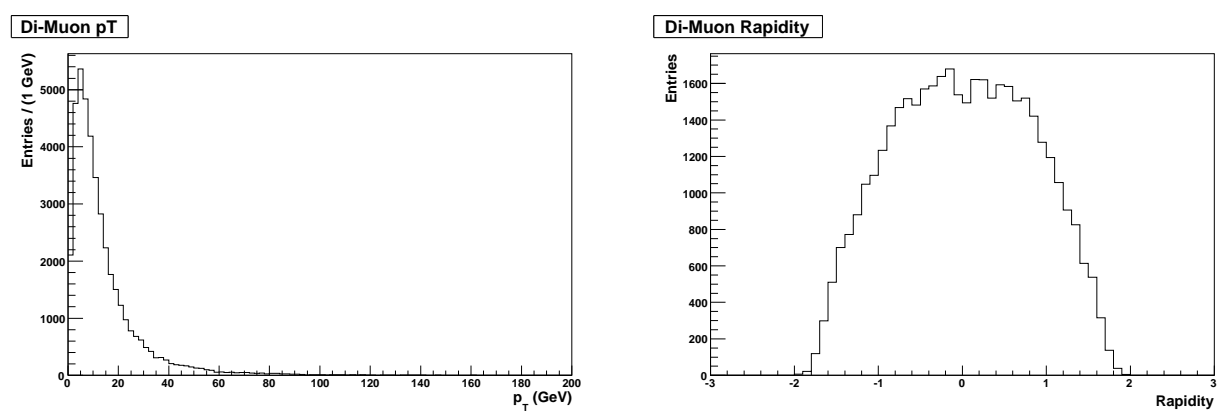

Figure 4.9: Di-Muon $p_{T}$ and Rapidity Distribution

\subsection{Trigger Selection}

The dataset used in this analysis is split in three trigger versions, V12,V13 and V14. Each trigger version has a requirement by level (L1,L2 and L3) ${ }^{2}$. The requirements are listed below [46]:

\footnotetext{
${ }^{2}$ See Appendix B for muon trigger definitions
} 
- "MUW_W_L2M3_TRK10" (V12):

Level1: tight scintillator and loose requirements in Wide region ${ }^{3}$.

Level2: medium muon with $p T>3 \mathrm{GeV}$

Level3: track with $p_{T}>10 \mathrm{GeV}$ (8 hits requirement).

- "MUH1_TK12_TLM12" (V13 and V14 ):

Level1: tight scintillator requirements in Wide region. Central track with $p T>10 \mathrm{GeV}$.

Level2: There are not requirements at Level 2

Level3: track with $p T>12 \mathrm{GeV}$ (10 hits requirement), Level 3 loose muon matched to the central track.

\subsection{Z Diffractive Event Selection}

Once the $\mathrm{Z}$ boson candidates have been selected, we have to apply diffractive cuts in order to obtain the $\mathrm{Z}$ diffractive sample. Single diffractive events are characterized by a small fraction of momentum loss fraction $(\xi<0.1)$ of the intact particle and low energy deposition where the beam particle remains intact (gap). Both definitions are explained below.

\subsubsection{Fraction of the Proton Momentum Loss}

In a single diffractive collision, one of the two incident particles remains intact (proton or antiproton). Due to the kinematics of the interaction the particle that remains intact looses a small fraction of its momentum. There are two methods to measure the fraction of the proton momentum loss $(\xi)$ : By measuring the final momentum of the intact particle or by reconstructing the fraction of the momentum loss by using the final state particles. In this analysis we use the second method.

We reconstruct $\xi$ [Fig 4.10] using Collin's equation [47]:

$$
\xi=\frac{\sum_{i}^{n} E_{T_{i}} e^{ \pm \eta_{i}}}{\sqrt{s}}
$$

\footnotetext{
${ }^{3}$ Wide region corresponds to muons into $|\eta|<1.6$. Muons out to this region don't pass the muon triggers requirement
} 
$E_{T_{i}}$ is either the transverse energy of the calorimeter cells or the transverse energy of the muons. $\eta$ is the pseudorapidity ${ }^{4}$ and $\sqrt{s}$ is the center of mass energy. The positive and negative signs ${ }^{5}$ imply that the sum is dominated by those particles of the highest rapidity, additionally, particles with large transverse momentum also contribute significantly. Therefore, muons from the $\mathrm{Z}$ boson decay will have a large contribution to this distribution given their large $E_{T}$ values.

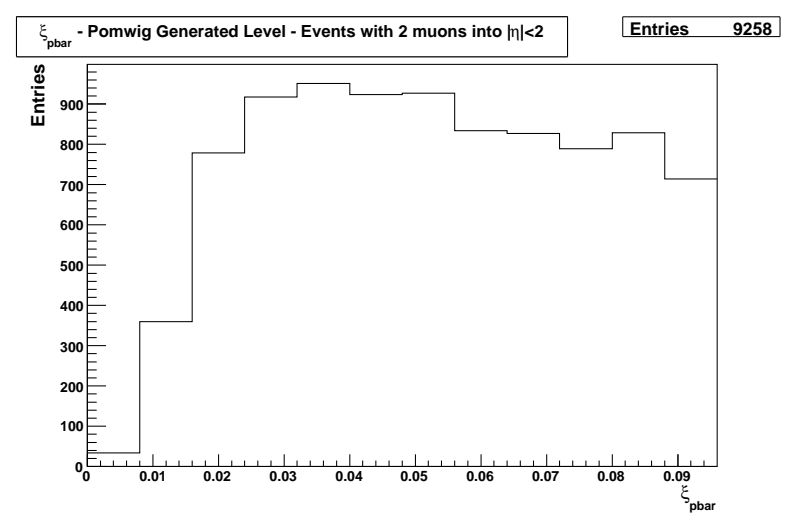

Figure 4.10: $\xi$ Reconstructed in the pbar side

Because $\xi$ is obtained from the dimuon contribution [Fig 4.11] and the calorimeter cell contribution, we have to analyze both contributions independently. The $p_{T}$ and $\eta$ of the muons are measured by the tracking detector with high precision. The muon detector has a geometrical acceptance for $|\eta|<2$, which corresponds to the central detector. The cell contribution comes from the calorimeter information.

\subsubsection{Gap Requirement}

Diffractive candidates are required to have a forward rapidity gap in the region $3.2<|\eta|<4.45$ and signal in the calorimeter cells up to $\eta<3.2$ in the proton direction and $\eta>-3.2$ in the antiproton direction. For Gap definition we demand no tracks and no energy deposition in the forward region $(3.2<|\eta|<4.45)$. The gap requirement reduces events with multiple interactions (events with more than one $p \bar{p}$ interaction in the same bunch crossing). The multiple interactions produce an error in the reconstruction of $\xi$ because there are cells with energy deposited

\footnotetext{
${ }^{4}$ At high energies, pseudorapidity $(\eta)$ and rapidity $(y)$ can be considered the same variable because $E \gg m$ for final state particles.

${ }^{5}$ We use $+\eta$ to identify the region of the scattered proton and $-\eta$ for the region of the scattered antiproton
} 


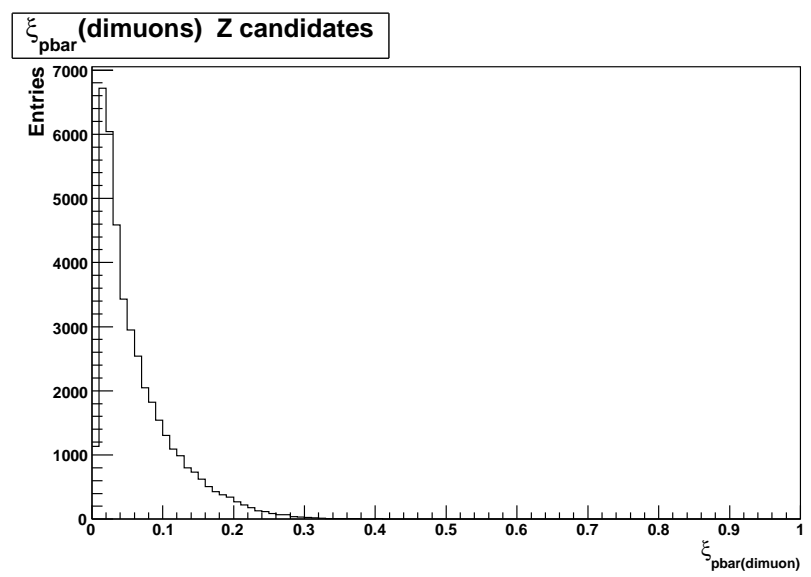

Figure 4.11: $\xi$ contribution from di-muons in the pbar side

from the additional interactions. In figure [Fig 4.12] we can see how the $\xi$ average increases with the number of primary vertices. Figure [Fig 4.13] shows the number of primary vertices for events with gap, only $9 \%$ of the events with gap have more than one vertex. In this analysis we require events with only one primary vertex, events excluded are taking into account in the efficiency determination. Figure [Fig 4.14] shows the $\xi$ distribution after diffractive cuts applied.

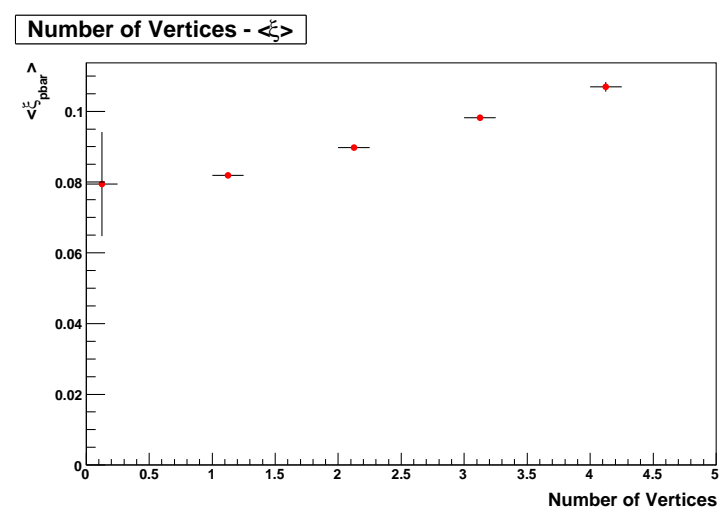

Figure 4.12: Number of primary vertices vs $\langle\xi\rangle$ 

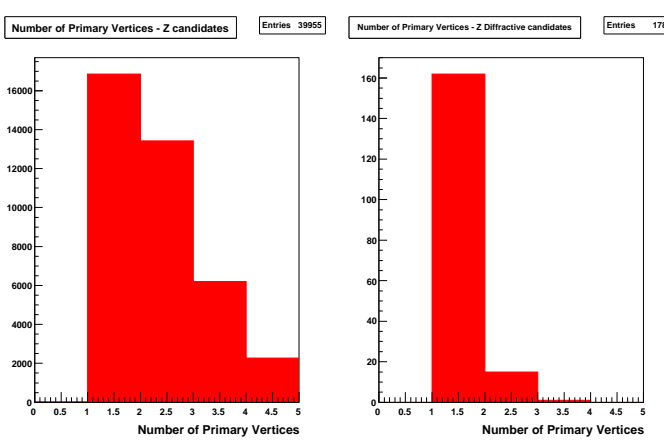

Figure 4.13: Primary vertex distribution - Before and After Diffractive cuts

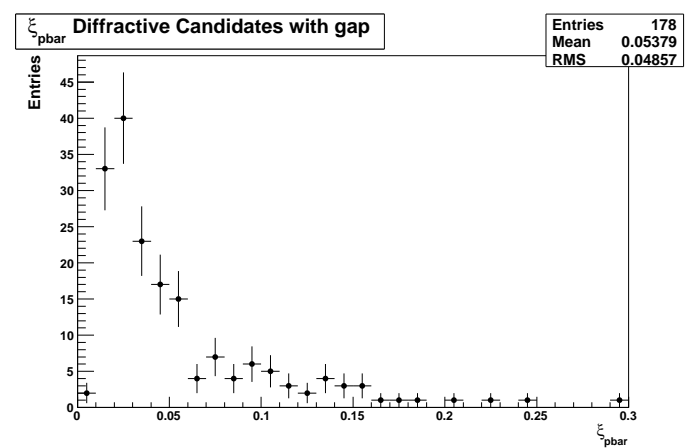

Figure 4.14: Fraction of antiproton momentum loss - Gap Requirement applied

\subsubsection{Cell Energy Thresholds}

In order to reconstruct $\xi$ from the calorimeter cells, we use the electromagnetic (Layers 1 to 3) and fine hadronic calorimeter (layers 7 to 11) [8]. We don't use the coarse hadronic calorimeter because its low energy resolution. The calorimeter has a geometrical acceptance of $|\eta|<4.45$ (Coarse Hadronic excluded), due to that, $\xi$ from cells is too sensitive to the energy deposited in the forward cells. This energy can come from particles that deposited their energy in the cells or it can be only noise. So, we have to determine the proper cell energy threshold in order to exclude noisy cells. Before to the object physics be reconstructed, noisy cells have been excluded event by event by using T42 and hot cell killer algorithms (They are explained in Appendix D). However, by using these algorithms, the ratio of 
noisy cells excluded are about $30 \%$ to $60 \%$ by event. So, the cell energy thresholds for applying,they will help to exclude the remnant noisy cells.

The electromagnetic and fine hadronic cells respond in a different way because they were constructed with different materials, therefore, we find thresholds separately for these two.

Figures [Fig 4.15] and [Fig 4.16] show the cell energy distribution by layer. We use three data samples for noise studies, they are: minimum_bias ${ }^{6}$, zero_bias 7 and Empty BX ${ }^{8}$ samples.
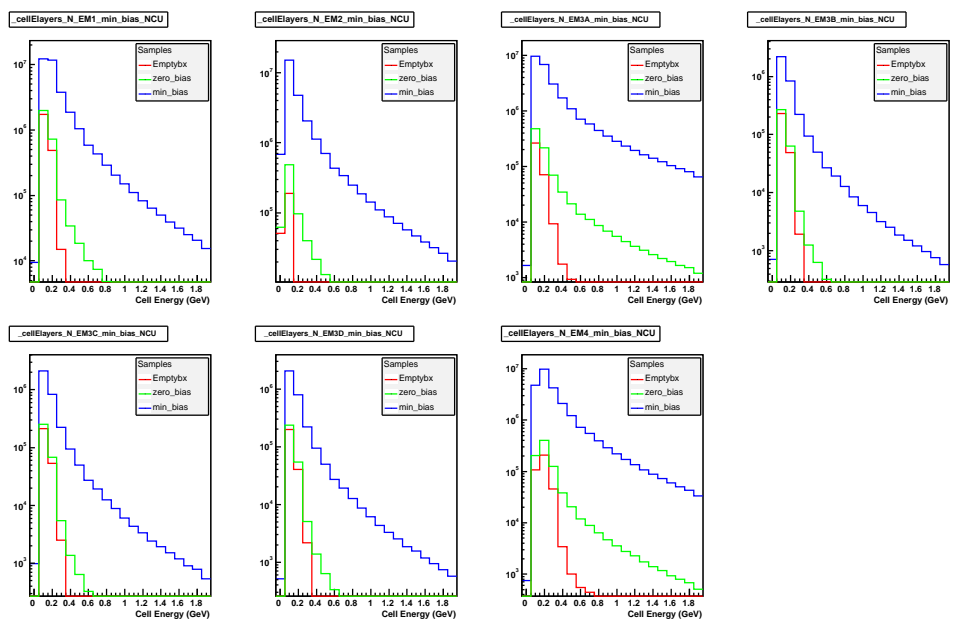

Figure 4.15: Energy cell distribution for Electromagnetic cells (Layers 1 to 7). Minimum bias, zero bias and emptybx sample.

The minimum bias sample has physics events, the zero bias sample includes physics signal and noise events and, the empty bx represents only noise. Under ideal conditions, the energy cell distribution for Empty BX sample should be zero because there are not interactions in this sample, however the cells have electronic noise and the energy distribution shows activity in the cells. Noisy cells with large $\eta$ value have a significant contribution to the $\xi$ distribution, the cell energy threshold reduces this source of contamination [Fig 4.17].

\footnotetext{
${ }^{6}$ minimum bias trigger requires beam crossing and N/S luminosity monitors above threshold in coincidence and NOT unsuppressed Calorimeter read out.

${ }^{7}$ Requires beam crossing (an accelerator condition) and NOT unsuppressed Calorimeter read out

${ }^{8}$ Data taken with zero bias trigger, and interactions are excluded by requiring no Luminosity Monitors fired and no vertices and jets
} 

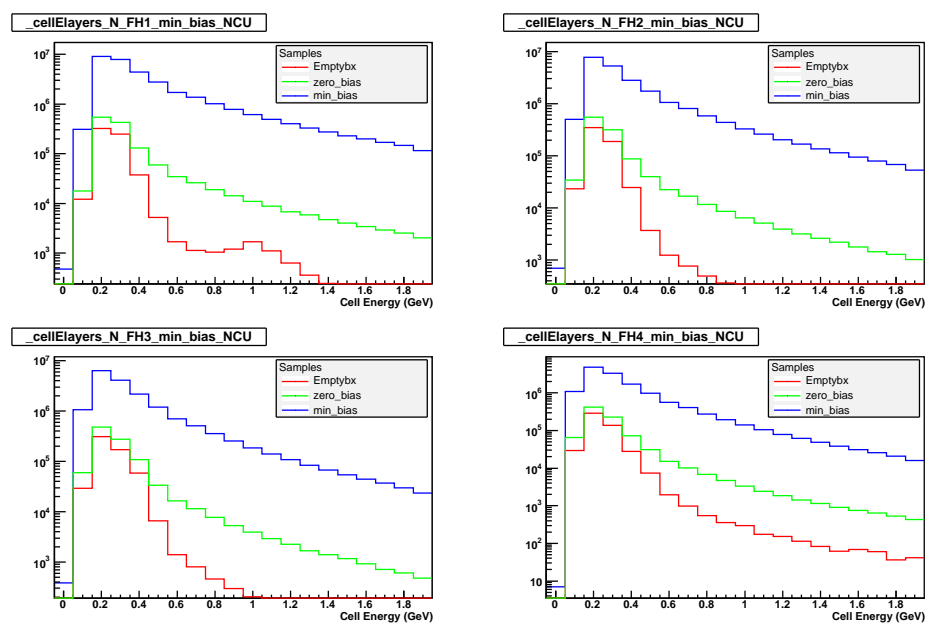

Figure 4.16: Energy cell distribution for Fine Hadronic cells (Layers 11 to 14). Minimum bias, zero bias and emptybx sample
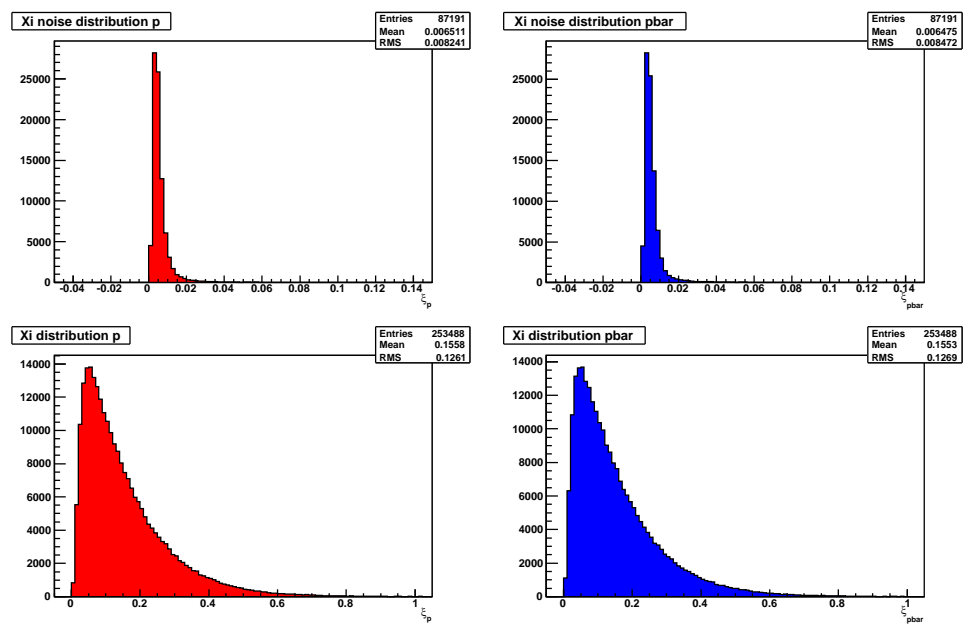

Figure 4.17: $\xi$ distributions - Physics signal and Noise for proton and antiproton side

The default cell thresholds used by $\mathrm{D} \varnothing$ experiment for data storing are $E M=$ $0.1 \mathrm{GeV}$ and $F H=0.2 \mathrm{GeV}$, we use the min bias sample for physics signal and Empty BX for noise and we reconstruct $\xi$ up to 0.001 at both sides of the 
calorimeter. We choose $\xi<0.001$ because this range is dominated by diffractive events. These distributions are normalized to the unit and they are compared [See Fig 4.18].

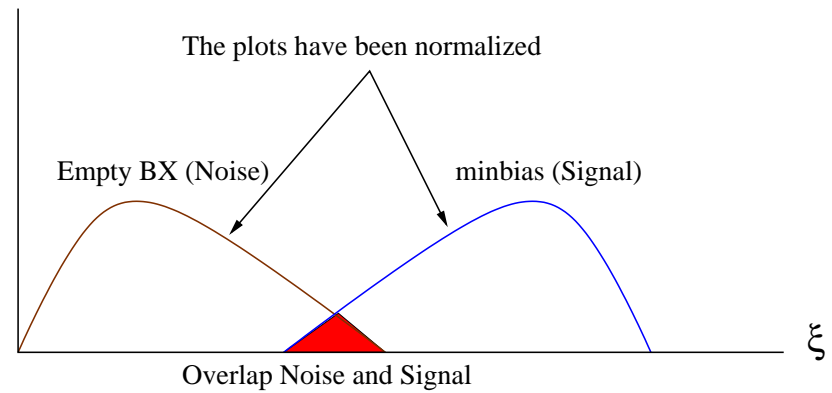

Figure 4.18: $\xi$ distributions - Empty BX (Noise) and MinBias(signal)

Because the plots have been normalized, the overlap area represents the noise fraction into the signal. These plots are made for each cell energy thresholds set, by starting with $0.15 \mathrm{GeV}$ for electromagnetic calorimeter cells and $0.25 \mathrm{GeV}$ for fine hadronic calorimeter cells. By increasing the thresholds in steps of $0.05 \mathrm{GeV}$ we can see that the noise excluded increases and the physics signal is slightly reduced. The best results were obtained for cells thresholds of: $E M=0.5 \mathrm{GeV}$ and $F H=0.6 \mathrm{GeV}$. With these thresholds we can exclude about $96 \%$ of noise at both sides of the calorimeter, as it is shown in figure [Fig 4.19], the noise that remains after the cell threshold cuts is later rejected with the other cuts to apply. Table 4.3 shows the thresholds used in [Fig 4.19] . The efficiency of these cuts are later determined by using montecarlo samples.

\begin{tabular}{|c||c||c||c||c||c||c||c||c||c||c||c||c|}
\hline \hline Number & 1 & 2 & 3 & 4 & 5 & 6 & 7 & 8 & 9 & 10 & 11 & 12 \\
\hline \hline EM Cell Thres. (GeV) & 0.15 & 0.2 & 0.25 & 0.3 & 0.35 & 0.4 & 0.45 & 0.5 & 0.55 & 0.6 & 0.65 & 0.7 \\
\hline FH Cell Thres. (GeV) & 0.25 & 0.3 & 0.35 & 0.4 & 0.45 & 0.5 & 0.55 & 0.6 & 0.65 & 0.7 & 0.75 & 0.8 \\
\hline
\end{tabular}

Table 4.3: Convention of numbers used for cell energy thresholds

\subsubsection{Choice of event kinematics}

Thresholds are applied to all calorimeter cells (Electromagnetic and Fine Hadronic) before applying the rapidity gap requirement and making the $\xi$ reconstruction.

We use tight cuts to obtain diffractive candidates. An event can be considered diffractive candidate if passes the following selection criteria: 

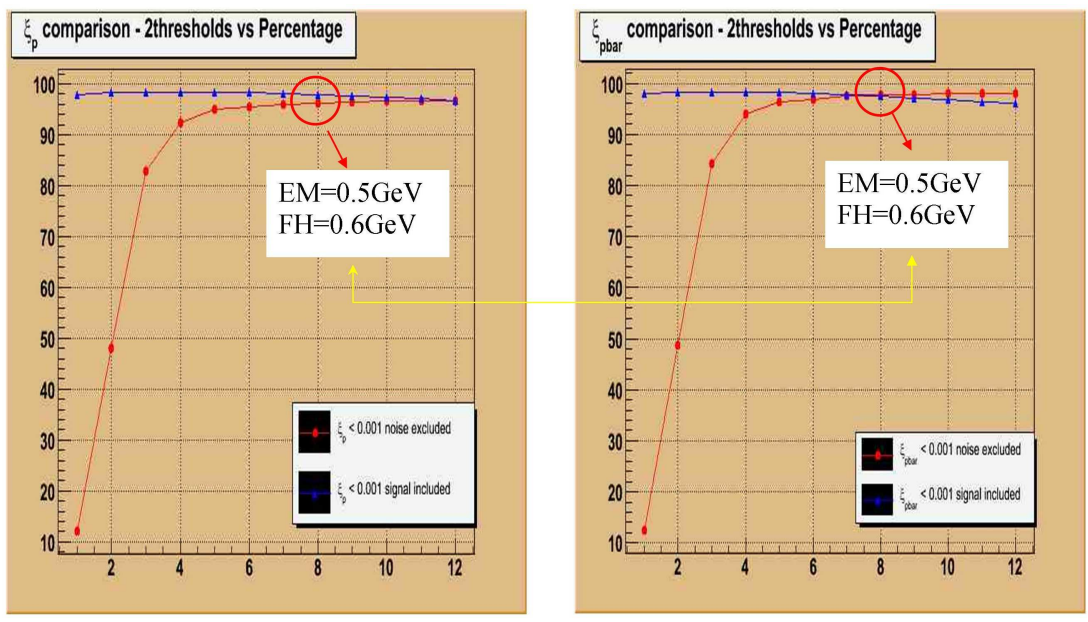

Figure 4.19: Thresholds for EM and FH calorimeter cells

- $\xi$ Requirement: Diffractive events are expected to be the dominant contribution at low $\xi$ values [43]. Events with $\xi<0.1$ pass this selection criteria.

- Gap Requirement: $\sum_{i=1}^{n} E_{i}^{\text {cells }}=0$ for forward calorimeter cells $(3.2<|\eta|<$ 4.45), where $E_{i}$ is the energy deposited on each cell in that region and, zero tracks reconstructed in the region $3.2<|\eta|<4.0{ }^{9}$. In previous analysis [45],[50] the Luminosity Monitors were used for rapidity gap requirement because this detector covers a region $2.7<|\eta|<4.4$. The Luminosity monitor information was based on three trigger bits:

- FastZ: Require both luminosity monitors firing in coincidence.

- AHalo: If LM South is hit before LM North.

- PHalo: If LM North is hit before LM South.

Only the first hit to each detector was recorded, so an event can fire at most only one of FastZ, AHalo or PHalo. The charge thresholds for the Luminosity Monitors were too low, which it does not represent problems for the tagging of non diffractive events because it demands both luminosity monitors to fire in coincidence (FastZ), this coincidence reduces the noise in the luminosity monitors. Single Diffractive Events demand a hit in only one luminosity monitor (North or South), however, due to the noise, both

\footnotetext{
${ }^{9}$ The tracking system has an acceptance up to $|\eta|<4$
} 
luminosity monitors in the region where the particle remains intact fired even when no energy was deposited in the calorimeter. There was not a way to set the charge thresholds offline.

However, the LM electronics was changed in October 2005 [51] and this new electronics allow to modify the charge thresholds offline. We know from previous results that the stadistics for diffractive events are too low, therefore, we need to use the highest stadistics available. Including the LM with the new electronics in this analysis would reduce the stadistics significantly (about $70 \%$ of the integrated luminosity) because the new LM electronics started on run number 211213 that corresponds to V14B trigger. For these reasons, we don't use the Luminosity monitors in this analysis. Figure [Fig 4.20] shows the $\xi$ distributions against sum of energy in the pbar side before and after applying the gap requirement. Montecarlo studies show that this tight requirement guarantees the best way to select diffractive events filtering out most of the non diffractive background (See Fig 4.33).
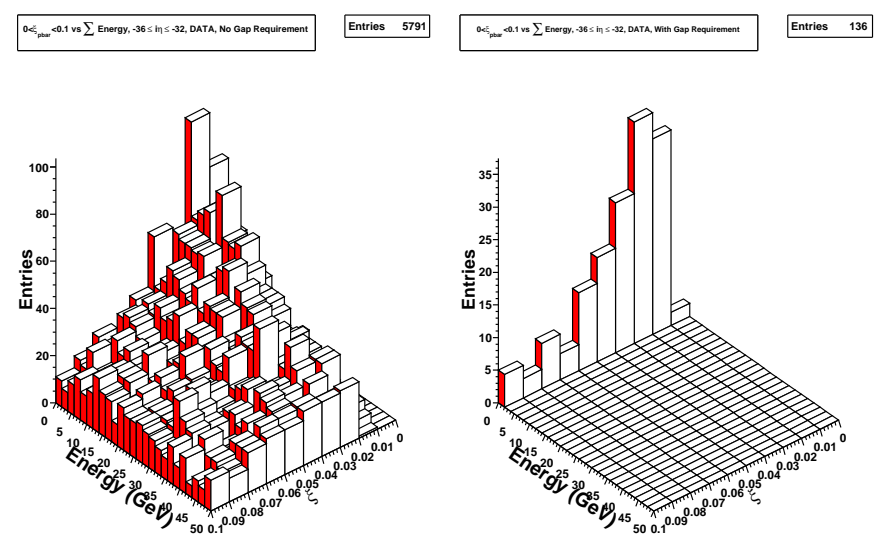

Figure 4.20: $\xi_{p b a r}$ against $\sum E$ for $-36<i \eta<-32$. Before and After Gap Requirement

- Single Diffractive Requirement: Single diffractive events are characterized by low energy deposition in the region where the particle remains intact (rapidity gap) and energy deposition in the calorimeter cells in the other side where the other beam particle was broken. So, we demand $\sum_{i=1}^{n} E_{i}^{\text {cells }}>5.0 \mathrm{GeV}$ at $2.0<|\eta|<4.45$ in the opposite region to the gap [Fig 4.21]. 


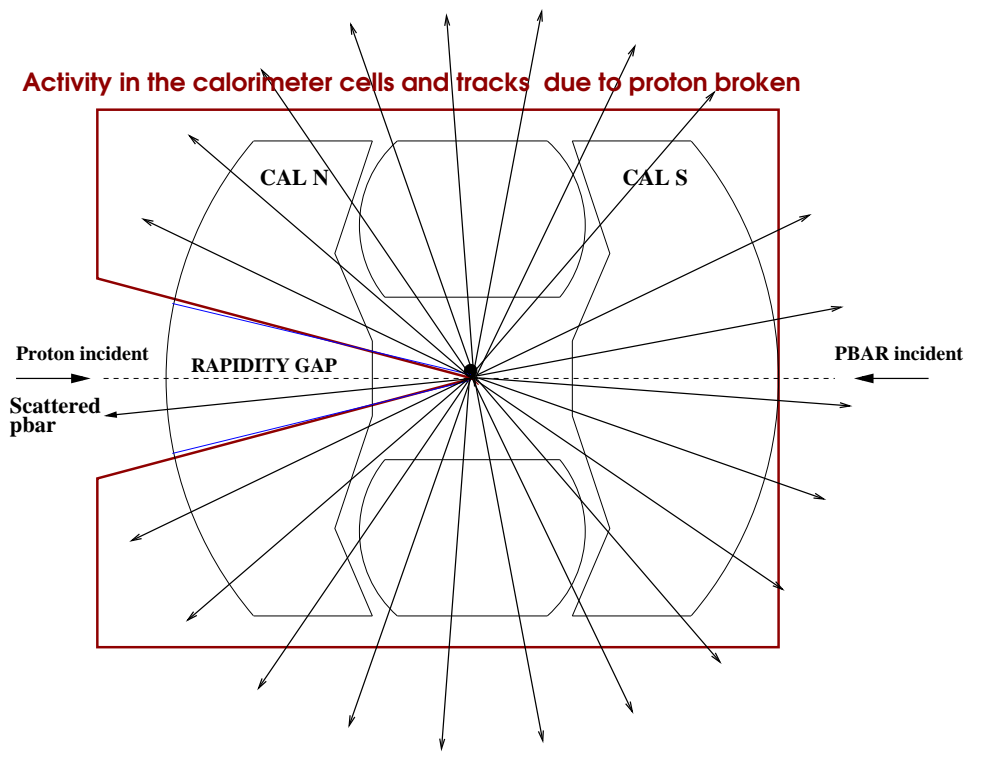

Figure 4.21: Single Diffractive signal at $\mathbf{D} \varnothing$

Figure [Fig 4.22] shows the $\xi_{\text {pbar }}$ and Rapidity distribution for events that pass all the requeriments before corrections and background substraction.
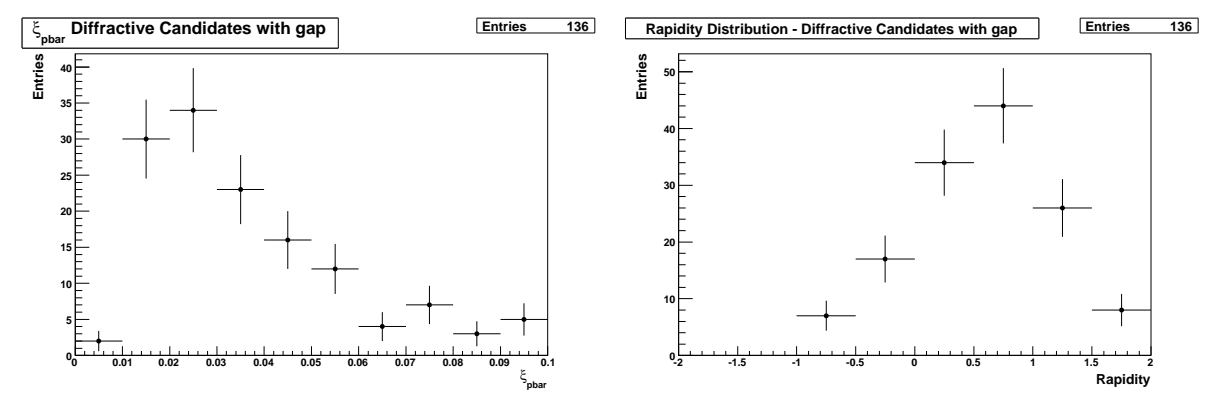

Figure 4.22: $\xi_{p b a r}$ and Rapidity distributions for Events that pass kinematical cuts 


\subsection{Montecarlo}

POMWIG [52] is the montecarlo used in this analysis to model diffractive $\mathrm{Z}$ scattering at the particle level. The simulated events are $p+\bar{p} \rightarrow \bar{p}+Z^{0} / \gamma^{*}+X$, in which the $\mathrm{Z}$ boson decays to two muons in a single diffractive event. POMWIG is based on HERWIG [53] event generator. To incorporate diffractive interactions, Pomwig modifies the hadron-hadron interactions by pomeron-hadron ${ }^{10}$ interactions. The pomeron is emitted by the antiproton beam, according to some flux formula, which in our case is the $\mathrm{H} 1$ diffractive structure function [54]. The Pomeron subsequently undergoes an interaction with the other proton beam [Fig 4.23]. In reality, either the proton or the antiproton could diffract, but currently POMWIG only allows the antiproton to emit the pomeron.

By default, the pomeron structure function used in this analysis involve a quark structure pomeron. This model consists of two quarks which share the pomeron momentum, $\frac{6}{4} \beta(1-\beta)$, where $\beta$ is the fraction of the pomeron momemtum that carries the parton . Diffractive $\mathrm{Z}$ production is also possible with a hard gluon pomeron, which shares its momentum between two gluons, $6 \beta(1-\beta)$ or a soft gluon pomeron, $6(1-\beta)^{5}$. According to a previous analysis [45], the soft gluon pomeron and the hard gluon pomeron is not expected to produce many diffractive $\mathrm{Z}$ bosons, the quark pomeron model produces by far the highest fraction (on the order of $20 \%$ higher) of diffractive $\mathrm{W}$ and $\mathrm{Z}$ bosons. For this reason in this analysis we use the quark pomeron model.

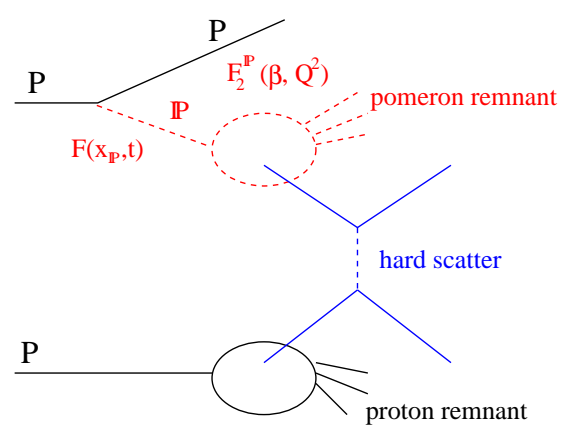

Figure 4.23: Single Diffractive events by POMWIG

The proton structure function chosen for our following Montecarlo studies was CTEQ6L [55]. This is a series of proton parton distribution functions (PDFs)

\footnotetext{
${ }^{10}$ In this analysis we use the pomeron model instead reggeon model for make diffractive interactions
} 
determined by the CTEQ collaboration ${ }^{11}$ through a global QCD analysis of fixed target and collider data [56].

POMWIG events were generated in the kinematical range $M_{Z \rightarrow \mu \mu}>40 \mathrm{GeV}$ (See Fig 4.24) and fraction of the antiproton momentum loss $\xi<0.1$. At generated level we know the final momentum for the antiproton that remained intact:

$$
\xi_{\text {pbar }}=1-\frac{p_{f}}{p_{l a b}}
$$

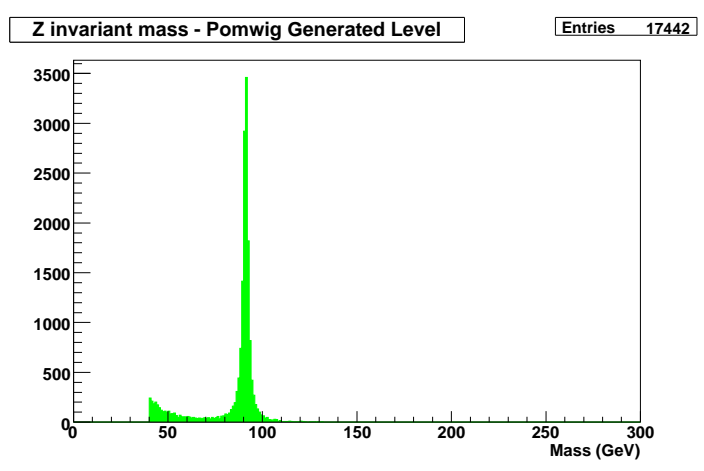

Figure 4.24: Z invariant mass - Pomwig at generated Level

$p_{f}$ is the antiproton final momentum and $p_{l a b}=980 \mathrm{GeV}$ is the antiproton momentum before the interaction [Fig 4.25].

POMWIG generates diffractive $\mathrm{Z}$ events without kinematic constraints. Event by event, the proton broken due to the interaction with the pomeron produces two muons with high $p_{T}$ [Fig 4.26] and soft particles (particles with low mass and energy ), many of these particles are created in the proton side $(\eta>0)$ and some particles are created in the antiproton side because the antiproton can lose up to $10 \%$ of its momentum during the interaction [Fig 4.27].

Pomwig simulation shows that there is a high fraction (about $40 \%$ ) of diffractive events with no rapidity gap (see Fig 4.28), most of the events generated in the forward region are soft pions; these events could be studied in an analisis based on the tagging of the intact particle, in the present analysis difractive events with no gap are excluded.

\footnotetext{
${ }^{11}$ CTEQ means: Coordinated Theoretical-Experimental project on QCD
} 


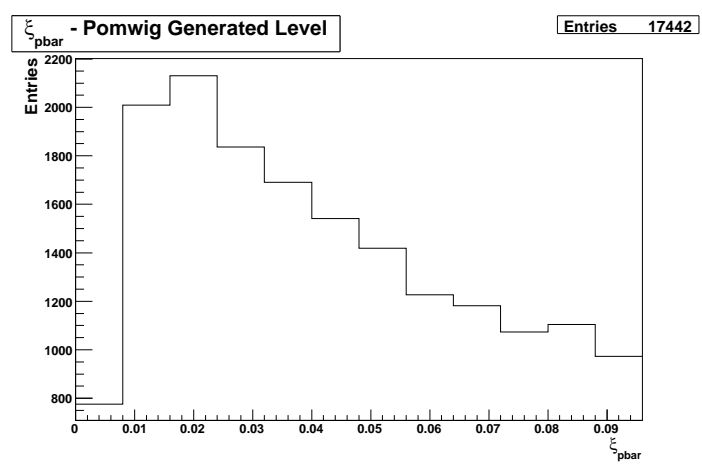

Figure 4.25: $\xi_{\text {pbar }}$ - Pomwig at generated Level

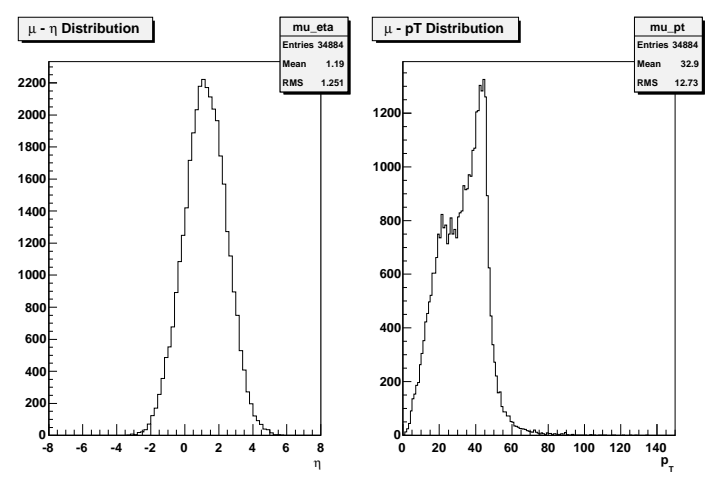

Figure 4.26: $\eta$ and $p_{T}$ distributions for muons from $\mathrm{Z}$ boson - POMWIG at generated level 


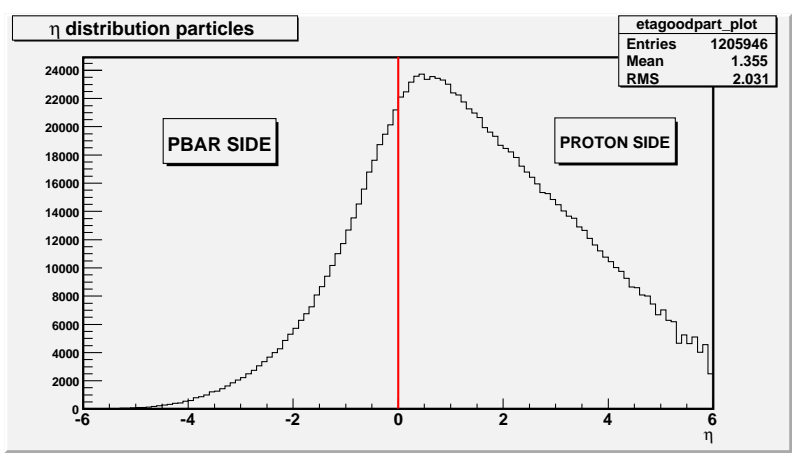

Figure 4.27: $\eta$ distribution for final state particles - POMWIG at generated level. The distribution shows that final particle states destroy the gap of most of diffractive events

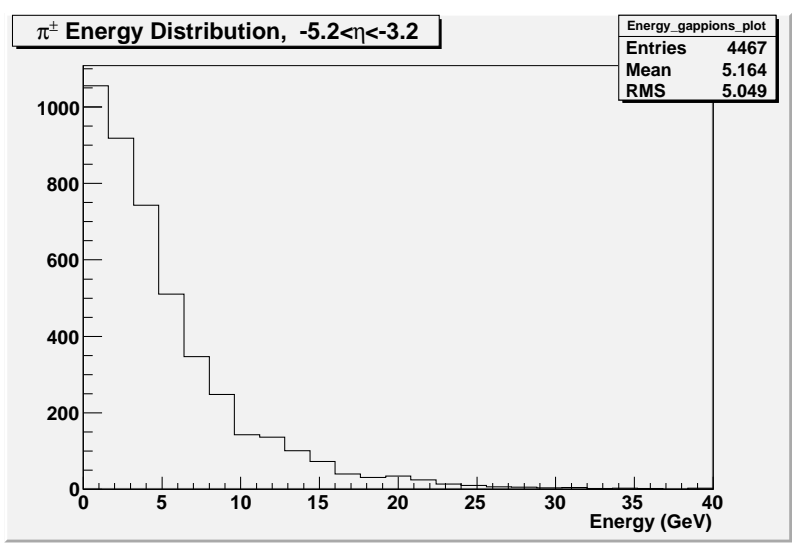

Figure 4.28: $\pi^{ \pm}$Energy distribution in the Gap region - POMWIG at generated level

The muon detector at $\mathrm{D} \varnothing$ can only detect muons in a region $|\eta|<2$, therefore, we have to exclude events generated with muons beyond this region. Figure [Fig $4.29]$ shows the events generated by satisfying this condition.

By comparing [Fig 4.25] and [Fig 4.29] we can see that in the region $0<$ $\xi_{\text {pbar }}<0.02$ the number of events is reduced drastically due to the geometrical acceptance of the muons, for this reason we exclude events with $\xi<0.02$, because these events would require a high acceptance correction and they would be very sensitive to any sistematic error in the acceptance determination [Fig 4.30]. 


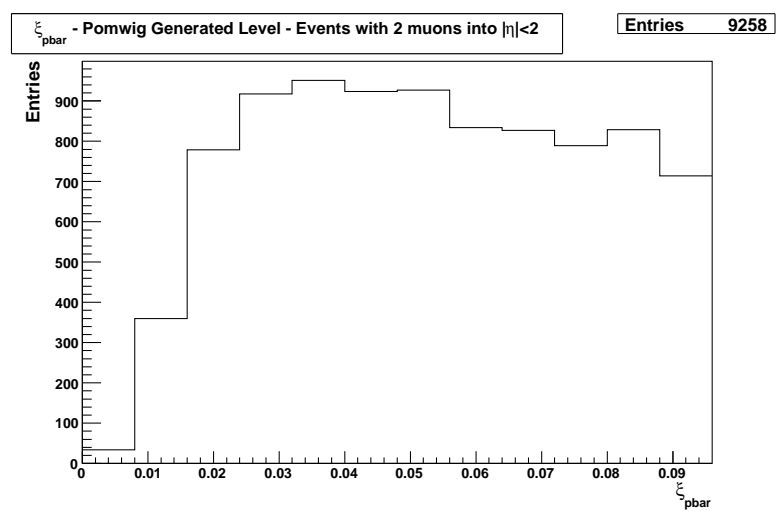

Figure 4.29: $\xi_{p b a r}$ - Events with two muons into $|\eta|<2$ - Pomwig at generated Level
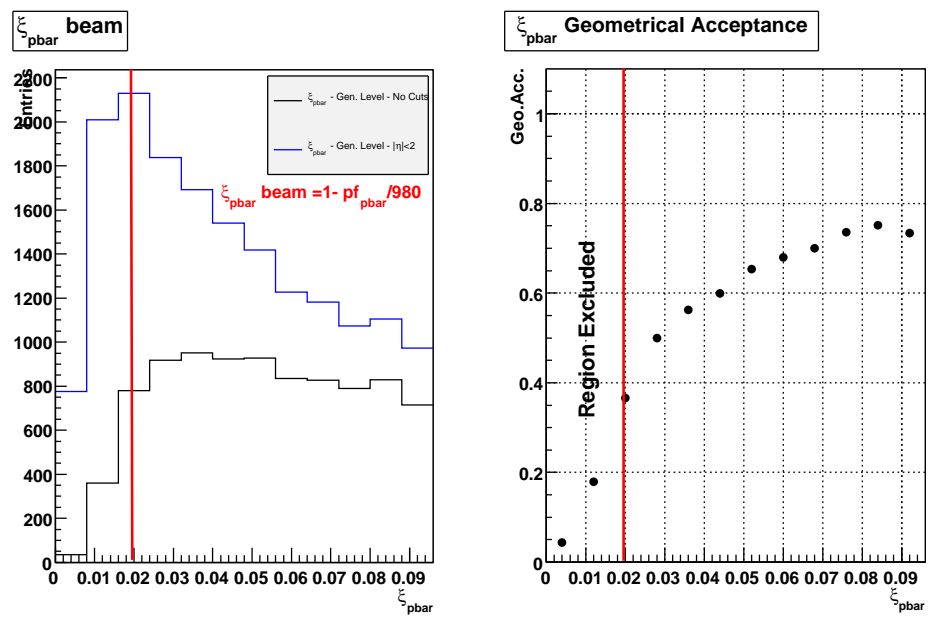

Figure 4.30: $\xi_{\text {pbar }}$ - Geometrical Acceptance - POMWIG at generated Level

Figure [Fig 4.31] shows the $\xi_{\text {pbar }}$ and Rapidity distribution that we expect from diffractive events after the gap requirement and acceptance cuts applied to POMWIG at generation level. We also obtain these distributions after full reconstruction in the $\mathrm{D} \varnothing$ detector in order to compare to real data.

The DØ detector simulation is based on two programs: DØgstar and DØsim [57]. DØgstar is a wrapper for GEANT [58], and determines how much energy is 

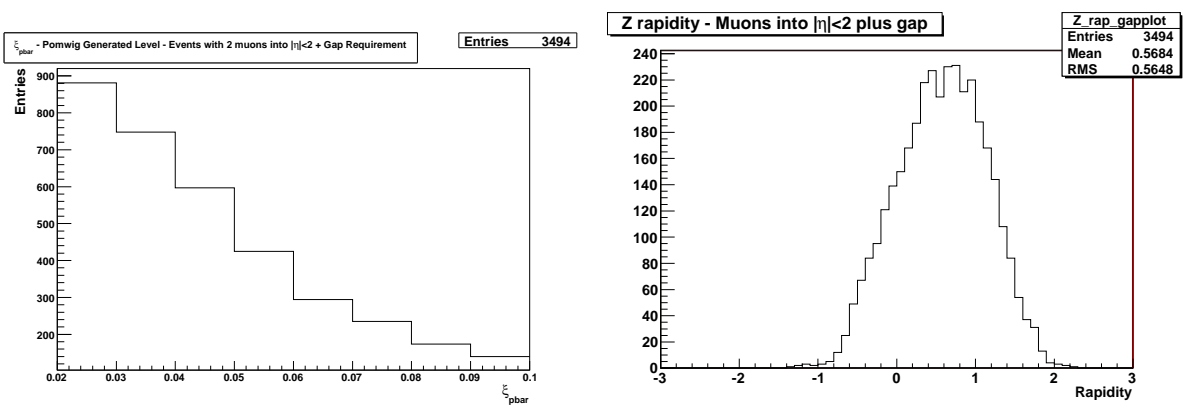

Figure 4.31: $\xi_{p b a r}$ and Rapidity Distribution - Events that pass all requirements - POMWIG at generated Level

deposited in the active areas of the detector. DØsim does electronics simulation and pileup of any additional minimum bias interactions that occur in the same crossing as the signal event [57]. After full simulation, DØreco reconstructs the physics objects (tracks, muons, electrons,etc) that are stored in thumbnails [59], thumbnails have the same format as the stored data. Simulation of trigger is not included into the full simulation.

After full simulation, we use the same analysis code that was used to filter the data with the diffractive cuts ${ }^{12}$. Figure [Fig 4.32] shows $p_{T}$, invariant mass, Rapidity and $\xi_{\text {pbar }}$ for $\mathrm{Z}$ reconstructed events after full simulation (no gap requirement was applied in these plots).

Figure [Fig 4.33] shows the sum of energy against $\xi_{\text {pbar }}$ for POMWIG events before and after applying the gap requirement. By comparing to the data sample [Fig 4.20] we can see that the requirement of low $\xi$ values is not a sufficient condition to find diffractive events, data shows some extra contribution which should be mainly low multiplicity non diffractive events. Our tight requirements for gap definition are justified by Pomwig simulation which shows that accepting events with $\sum E=0$ guarantees to keep a good fraction of diffractive events, and rejecting most of the non diffractive contamination.

Figure [Fig 4.34] shows the $\xi_{\text {pbar }}$ distribution for POMWIG events with the gap requirement and $\xi_{\text {pbar }}$ acceptance cuts applied.

\footnotetext{
${ }^{12} \mathrm{CAF}$ is the standard format used for analysis in the $\mathrm{D} \varnothing$ collaboration [60]
} 

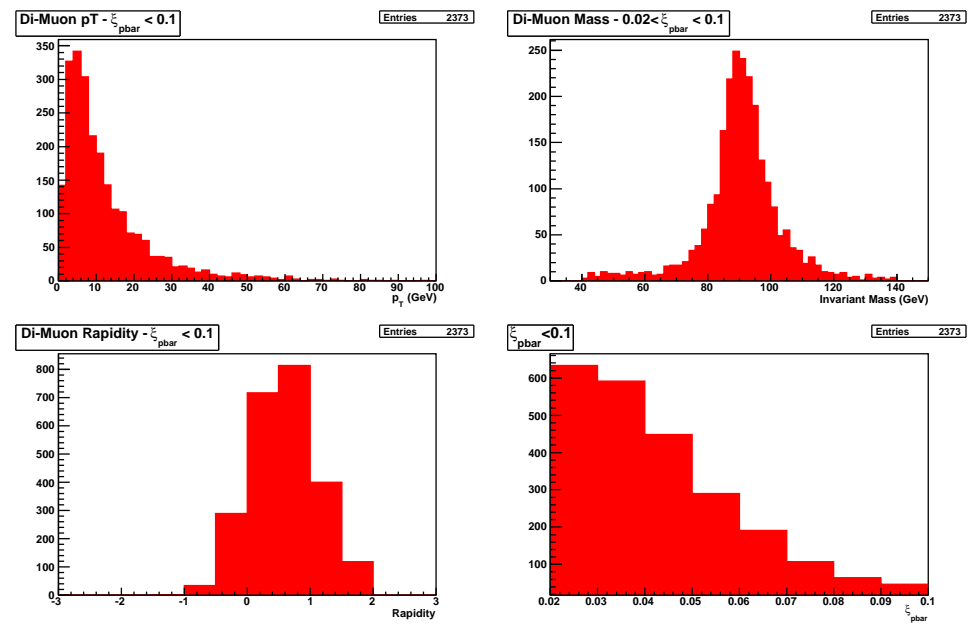

Figure 4.32: POMWIG plots after Full Simulation. $P_{t}$ and Rapidity distribution, dimuon mass and $\xi_{\text {pbar }}$

\subsection{Background Subtraction Method}

When we apply diffractive cuts some non-diffractive events pass these cuts, so, we have to use a method in order to substract this source of background. To
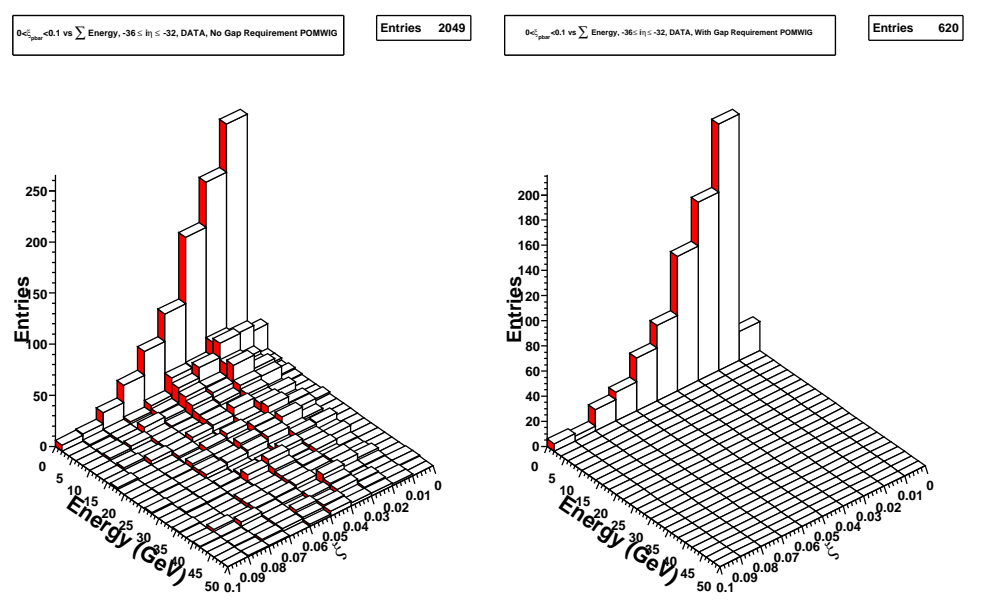

Figure 4.33: $\sum E$ against $\xi_{p b a r}$ - POMWIG events before and after gap requirement 

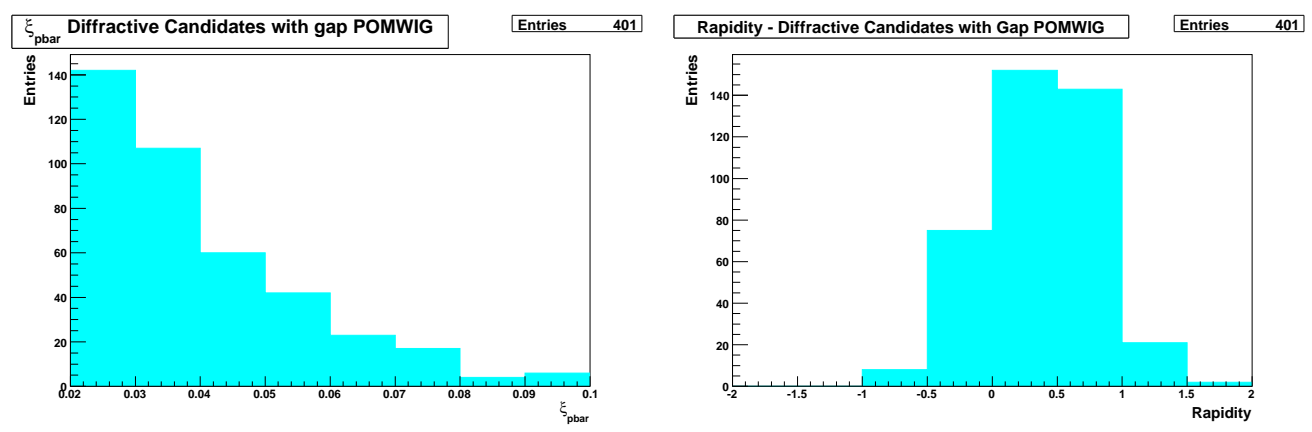

Figure 4.34: $\xi_{\text {pbar }}$ and Rapidity distributions for POMWIG events that pass all diffractive cuts

do this, we use PYTHIA [61] a non-diffractive montecarlo. The PYTHIA sample generated corresponds to $Z^{0} / \gamma * \rightarrow \mu^{+} \mu^{-}$that has been passed through full simulation. Figure [Fig 4.35] shows the kinematical variables for $\mathrm{Z}$ reconstructed from PYTHIA.
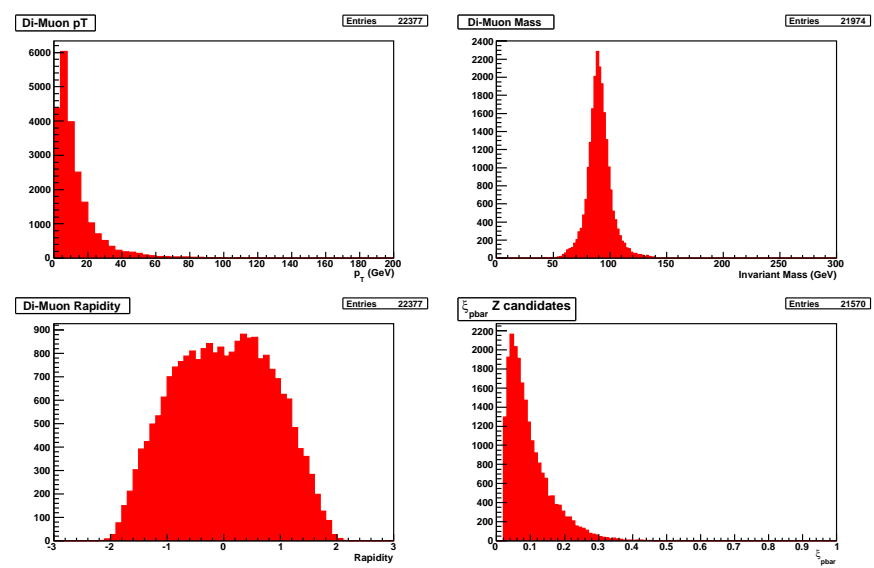

Figure 4.35: Z kinematical Variables - PYTHIA. $P_{t}$ and Rapidity ditribution, Di-muon mass and $\xi_{p b a r}$ distribution

We apply diffractive cuts to the PYTHIA sample for $\xi_{p b a r}$ and Rapidity distributions in order to know how many events pass these selection cuts, [Fig 4.36].

Initially, we have to normalize the samples to the integrated luminosity: data, POMWIG and PYTHIA. The integrated luminosity for a data sample is given by: 

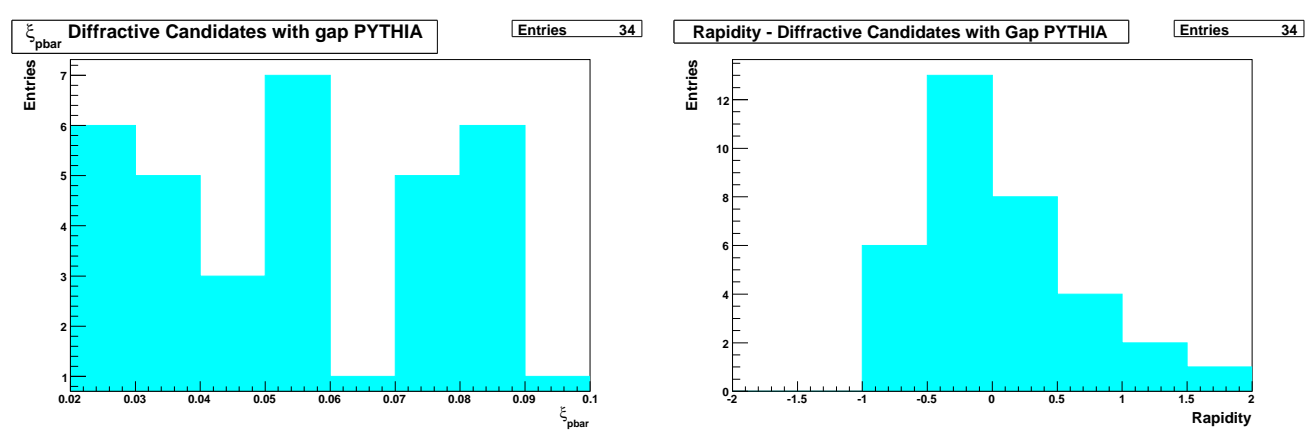

Figure 4.36: $\xi_{\text {pbar }}$ and Rapidity distributions for PYTHIA events that pass all diffractive cuts

$$
\int \mathcal{L} d t=\frac{N_{p \bar{p}}}{\varepsilon_{L M} \sigma_{p \bar{p}}}
$$

where $N_{p \bar{p}}$ is the number of inelastic $p \bar{p}$ interactions, $\sigma_{p \bar{p}}$ is the total inelastic $p \bar{p}$ cross section and $\varepsilon_{L M}$ is the efficiency of the Luminosity Monitor, which includes the geometrical acceptance as well as the efficiency to detect charged particles within the acceptance. Luminosity Monitors were described in the D $\varnothing$ detector chapter. $\sigma_{p \bar{p}}$ was measured by CDF [62] and E811 [63] experiments at $\sqrt{s}=$ $1.8 \mathrm{TeV}$. The two results are combined to give a value which is extrapolated to $\sqrt{s}=1.96 \mathrm{TeV}$ to a value of $60.7 \pm 2.4 \mathrm{mb}$ [64]. The integrated luminosity is calculated for each trigger version [Table 4.1]. For PYTHIA the integrated luminosity is:

$$
\int \mathcal{L} d t=\frac{N}{\sigma}
$$

where $N$ is the number of events generated by PYTHIA $(100.8 K)$ and $\sigma$ is the cross section for $Z / \gamma^{*} \rightarrow \mu^{+} \mu^{-}$, this value is $175.5 p b$. For POMWIG we normalize to the number of events generated $(22.5 K)$ and multiply by the normalization constant that is obtained by fitting Pomwig to diffractive sample, the normalization constant for POMWIG will be related with the cross section for diffractive Z production decaying to two muons.

The data sample is split in three trigger versions. Each trigger version is divided in subversions, table [Table 4.4] shows the subversions by trigger and the integrated luminosities. Taking into account the subversions by trigger, the data 


\begin{tabular}{|c||c||c||c||c||c||c||c||c||c|}
\hline \multicolumn{1}{|c|}{ SUBVERSION TRIGGERS } \\
\hline \hline v12a & v12b & v12c & v13a & v13c & v13d & v13e & v14a & v14b & v14c \\
\hline 40.55 & 88.38 & 95.21 & 16.85 & 124.53 & 81.17 & 107.93 & 137.04 & 68.54 & 59.59 \\
\hline
\end{tabular}

Table 4.4: Subversions by Trigger Versions - $\int \mathcal{L} d t$ in $p b^{-1}$

sample is divided into 10 parts. Each part is analyzed independently because we have to apply correction factors for $\eta$ and $p_{T}$ of the muons for each trigger version.

Because we did not simulate triggers in Pythia we need to take into account any trigger effect, to do this we use non diffractive events. We define non-diffractive cuts that we apply to the pythia sample and data. We fit PYTHIA sample to DATA by using the $\chi^{2}$ minimization method, according the formula:

$$
\chi^{2}=\sum_{i=1}^{n}\left(\frac{N_{i}-C * P y t_{i}}{\sigma_{i}}\right)^{2}
$$

where $i$ run over each bin in the $\xi_{\text {pbar }}$ distribution after non-diffractive cuts. $N_{i}$ and $P y t_{i}$ correspond to data and PYTHIA respectively by bin. The normalization constant $C$ is associated to the trigger effect. Figure [Fig 4.37] shows the normalization for V13 trigger, same results were obtained in V12 and V14 triggers, Table 4.5 shows the normalization constant obtained by trigger version for PYTHIA.

\begin{tabular}{|c||c|}
\hline Trigger & Constant \\
\hline v12a & $1.09 \pm 0.06$ \\
\hline v12b & $0.91 \pm 0.04$ \\
\hline v12c & $0.75 \pm 0.03$ \\
\hline v13a & $0.70 \pm 0.07$ \\
\hline v13c & $0.75 \pm 0.03$ \\
\hline v13d & $0.65 \pm 0.03$ \\
\hline v13e & $0.63 \pm 0.03$ \\
\hline v14a & $0.64 \pm 0.02$ \\
\hline v14b & $0.56 \pm 0.03$ \\
\hline v14c & $0.57 \pm 0.04$ \\
\hline
\end{tabular}

Table 4.5: Normalization constants for PYTHIA

The $\xi_{p b a r}$ distribution for PYTHIA sample that pass all the diffractive cuts is multiplied bin to bin by the respective normalization constant, this final PYTHIA 

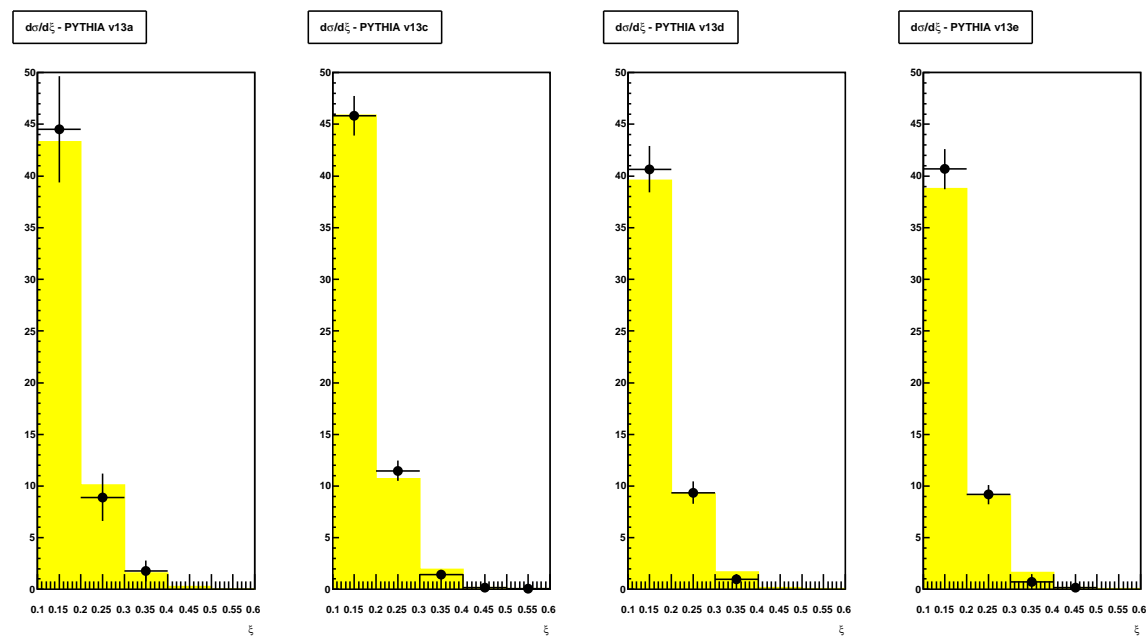

Figure 4.37: $\xi_{p b a r}$ non diffractive cuts DATA and PYTHIA

plot corresponds to the background. Figure [Fig 4.38] shows the $\xi_{\text {pbar }}$ distributions for diffractive signal and background.

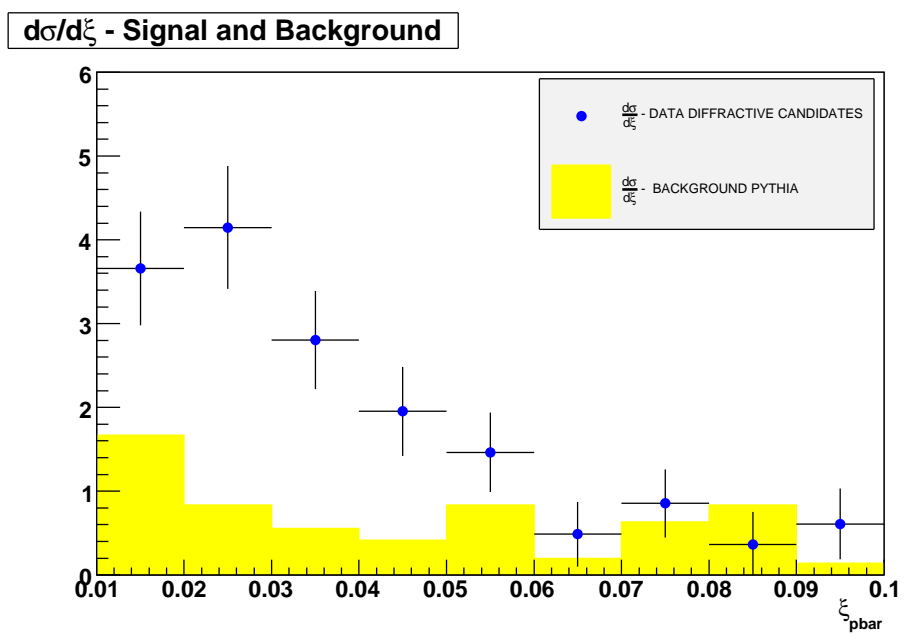

Figure 4.38: $\xi_{\text {pbar }}$ Distribution SIGNAL and Background

After background subtraction, we can compare the diffractive sample with the diffractive montecarlo (POMWIG). Figure [Fig 4.39] shows that the POMWIG 
montecarlo models reasonably well $\left(\chi^{2} / d o f=1.05\right)$ the signal obtained from the data.

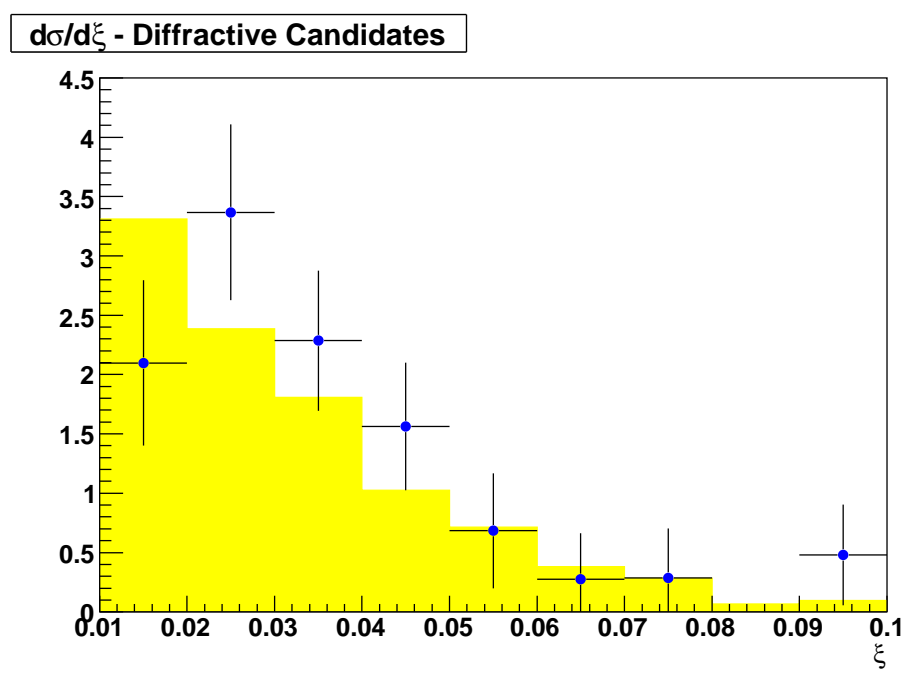

Figure 4.39: $\xi_{p b a r}$ Distribution - Diffractive Candidates and POMWIG

We have to correct the measured $\xi$ value to obtain the real $\xi$ value. To do this, we use the diffractive montecarlo sample. At generation level we know $\xi$ because we have information about the final momentum from the scattered antiproton and the initial momentum, so, we use equation Eq. 4.2. But, when the montecarlo sample is passed through the full $\mathrm{D} \varnothing$ simulation, we loose the information of the leading particle, so, we use the equation Eq. 4.1 in order to reconstruct $\xi$ for events that pass the gap requirement.

Figure [Fig 4.40] shows $\Delta \xi=\xi_{\text {gen }}-\xi_{\text {reco }}$ (Left side) and $\xi_{\text {reco }}$ against $\xi_{\text {gen }}$ (Righ Side).

We fit the distribution $\xi_{\text {gen }}$ versus $\xi_{\text {reco }}$ with the polinomial function to find a way to obtain real $\xi$ from $\xi$ observed:

$$
\xi_{\text {corr }}=0.006+1.426 \xi_{\text {reco }}-5.334 \xi_{\text {reco }}^{2}
$$

Table 4.6 shows the $\xi$ reconstructed and the $\xi$ corrected after applying correction factors.

After correction factors applied for $\xi$, we compare the $\xi$ corrected with the generated. Figure [Fig 4.41] shows $\Delta \xi=\xi_{\text {gen }}-\xi_{\text {corr }}$ (Left side), and $\xi_{\text {corr }}$ against $\xi_{\text {gen }}$ (Right). Both plots show that after correction we get the real value for $\xi$. 

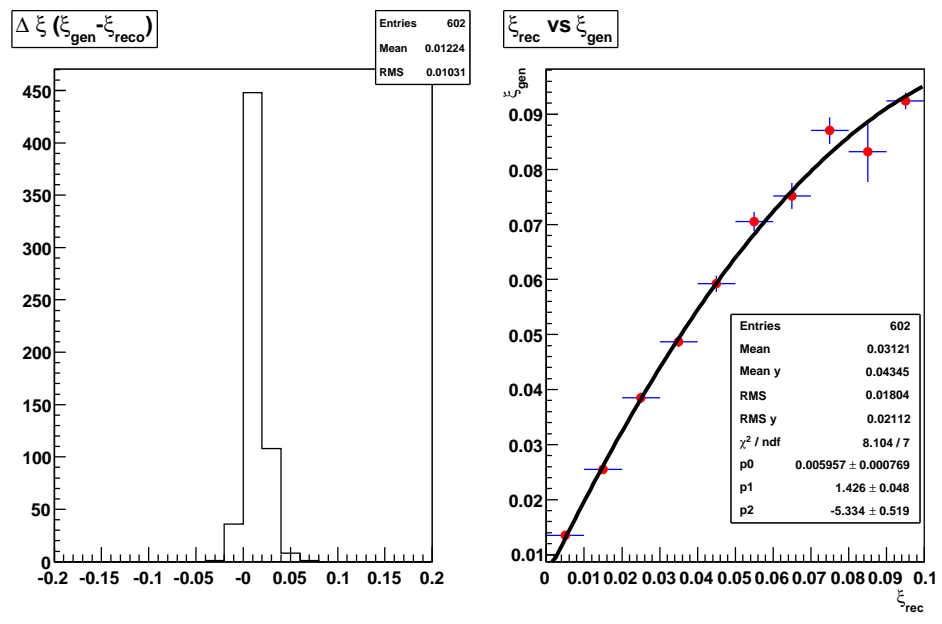

Figure 4.40: $\Delta \xi$ (Left) and $\xi_{\text {reco }}$ against $\xi_{\text {gen }}$ (Right). The 2D plot is fitted to the polinomial function to ajust the $\xi_{\text {reco }}$ to the $\xi_{\text {gen }}$

Figure [Fig 4.42] shows the $\xi$ distribution for diffractive candidates compared to POMWIG results after corrections.

In order to obtain the Rapidity distribution for diffractive candidates we use a similar procedure as used for $\xi$. Figure [Fig 4.43] shows the rapidity distributions for data and PYTHIA, both samples have been normalized by integrated luminosity.

Pythia also has been corrected to take into account trigger effects, by using

\begin{tabular}{|c||c|}
\hline \hline$\xi$ Reconstructed & $\xi$ Corrected \\
\hline 0.015 & 0.0262 \\
\hline 0.025 & 0.0383 \\
\hline 0.035 & 0.0494 \\
\hline 0.045 & 0.0594 \\
\hline 0.055 & 0.0683 \\
\hline 0.065 & 0.0762 \\
\hline 0.075 & 0.0829 \\
\hline 0.085 & 0.0887 \\
\hline 0.095 & 0.0933 \\
\hline
\end{tabular}

Table 4.6: $\xi$ reconstructed and $\xi$ corrected, correction factors applied 

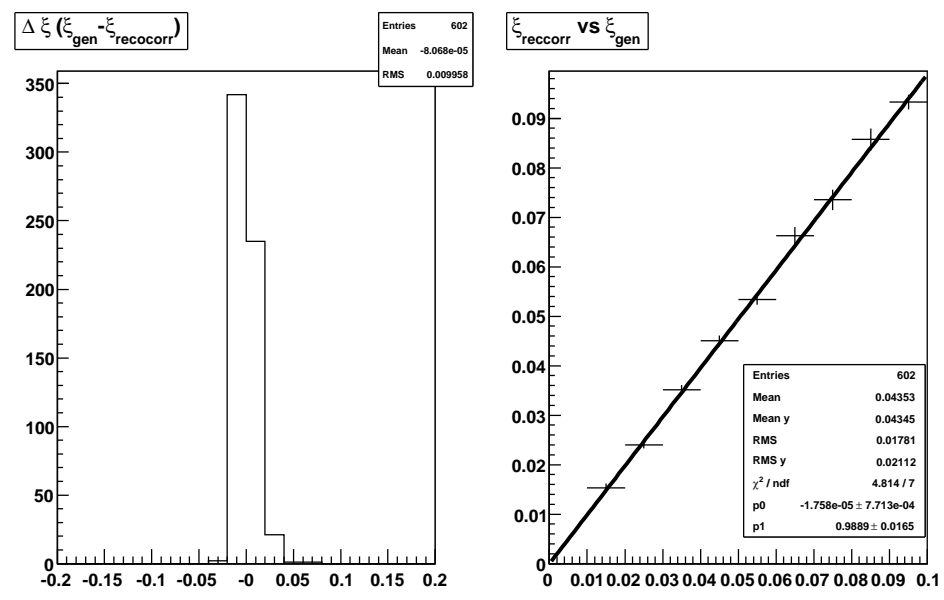

Figure 4.41: $\Delta \xi$ (Left) and $\xi_{\text {corr }}$ against $\xi_{\text {gen }}$ (Right). The 2D plot is fitted to the linear function, the slope is about 1 , it shows the correspondence one by one between both variables

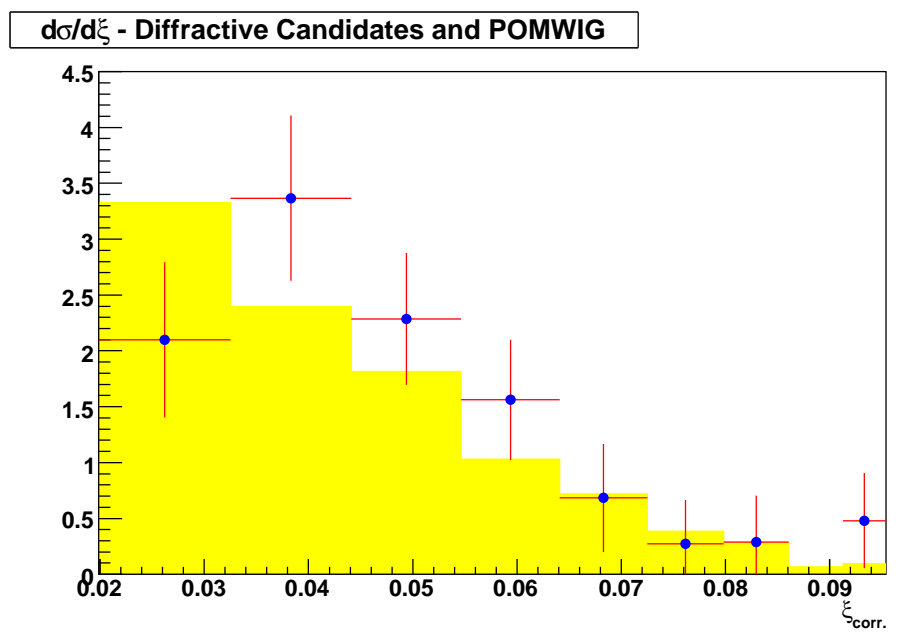

Figure 4.42: $d \sigma / d \xi$ diffractive candidates and POMWIG. The mean value by bin corresponds to the $\xi$ corrected. (See Table 4.6)

the normalization constants obtained from the comparison of events that passed non diffractive cuts in data and Pythia. We compare data after background sub- 


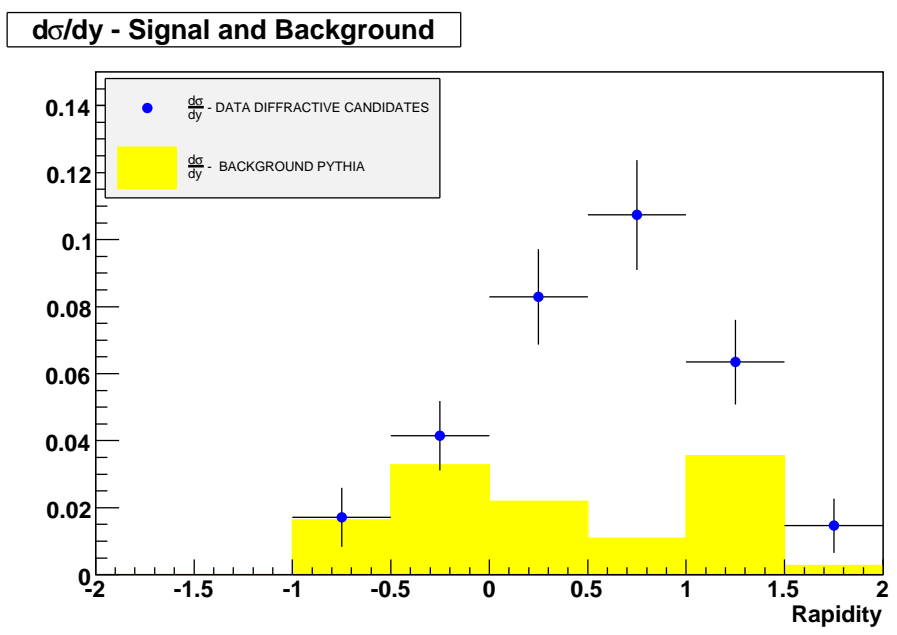

Figure 4.43: Rapidity Distribution SIGNAL and Background

traction, to POMWIG prediction in figure [Fig 4.44], we obtain a reasonable agreement $\left(\chi^{2} /\right.$ dof $\left.=1.05\right)$.

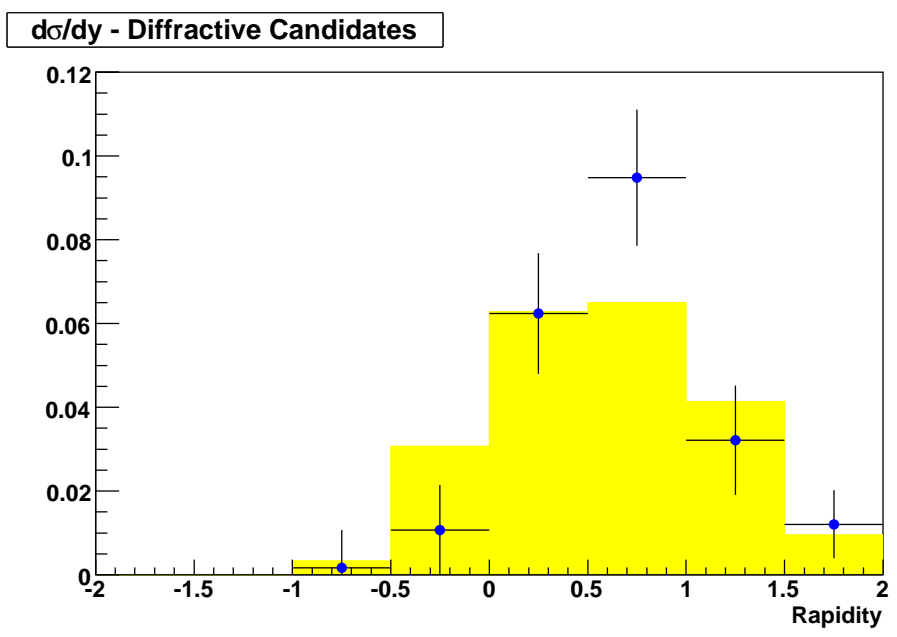

Figure 4.44: $d \sigma / d y$ Diffractive Candidates and POMWIG 


\subsection{Diffractive Cross section for $\mathrm{Z}$ boson decaying in two muons}

In this section we describe the method used to obtain the cross section multiplied by branching ratio for diffractive $\mathrm{Z}$ bosons with gap decaying in two muons and the fraction of diffractive $\mathrm{Z}$ bosons with gap with respect to all $\mathrm{Z}$ bosons.

\subsubsection{Corrections to obtain the Cross Section}

The Cross Section multiplied by the branching ratio $(\sigma \times B r)$ for Diffractive $\mathrm{Z}$ events with gap is calculated from the number of events that passed all diffractive cuts divided by the integrated luminosity. The value obtained should be corrected by various factors due to inefficiencies of the detector and background contamination. The equation used is:

$$
\begin{aligned}
& \sigma \times B r\left(Z / \gamma^{*} \rightarrow \mu^{+} \mu^{-}, 0.02<\xi<0.1\right)= \\
& \frac{N}{\int \mathcal{L} d t} \cdot \frac{\left(1-f_{b b}-f_{\text {cos }}\right)\left(1-f_{\tau \tau}\right)\left(1-f_{W}\right)}{\varepsilon_{M C} \varepsilon_{\text {opp } q} \varepsilon_{\text {isol }} \varepsilon_{\text {cosmic }}}
\end{aligned}
$$

$N$ being the number of diffractive candidates with gap, $\int \mathcal{L} d t$ the integrated luminosity, $f_{b b}, f_{c o s}, f_{\tau \tau}, f_{W}$ are correction factors due to background contamination and $\varepsilon_{g a p}, \varepsilon_{M C}, \varepsilon_{o p p_{-} q}, \varepsilon_{i s o l}, \varepsilon_{\text {cosmic }}$ correspond to efficiencies calculated for $\mathrm{Z}$ boson inclusive production.

\section{Number of Events}

The number of events that pass the $\mathrm{Z}$ boson diffractive selection with an intact antiproton are 134 events before to background subtraction. After subtracting background the number of events is reduced to: $87.1 \pm 13.1$. However, the sample corresponds to single diffractive events with an intact antiproton. The number of diffractive events is multiplied by 2 to take into account the case of diffractive $\mathrm{Z}$ with the intact proton. The total number of single diffractive events in this analysis is $174.2 \pm 26.3$.

\section{Integrated Luminosity}

The integrated luminosity for this analysis is $\int \mathcal{L} d t=819.8 p b^{-1}$. This value was obtained taking the luminosity recorded in the data sample for each trigger version (see Table 4.4). 


\section{Efficiency of MonteCarlo}

The Montecarlo efficiency $\left(\varepsilon_{M C}\right)$ combines the geometrical acceptance of the muon detector, the mass threshold from the dimuon candidate and the gap requirement. To do this, we use the $Z / \gamma^{*} \rightarrow \mu^{+} \mu^{-}$diffractive montecarlo sample (POMWIG) at generation level and we demand events with two muons into geometrical acceptance of the muon detector $\left|\eta_{\text {det }}\right|<2$, the Di-muon mass should be $M_{\mu \mu}>40 \mathrm{GeV}$, the fraction of the antiproton momentum loss $0.02<\xi<0.1$ and no particles in $-4.45<\eta<-3.2$ (gap requirement). We count how many events pass the diffractive requirements after full simulation. The ratio of the number of events after full simulation that pass diffractive cuts divided by the number of events at generation level with gap is the montecarlo efficiency, the value obtained is,

$$
\varepsilon_{M C}=0.052 \pm 0.002
$$

\section{Other Efficiencies and backgrounds}

We also include in our analysis, efficiencies and backgrounds that are taken into account in the $\mathrm{Z}$ boson inclusive studies approved by $\mathrm{D} \varnothing$ collaboration [44], these efficiencies and corrections are described in detail in Appendix C. Table 4.7 shows the correction factor and efficiencies applied to calculate the $\mathrm{Z}$ diffractive cross section.

\begin{tabular}{|c||c|}
\hline Efficiences/Background & Value \\
\hline$\varepsilon_{i s o l}$ & $0.999 \pm 0.001$ \\
\hline$\varepsilon_{\text {cosmic }}$ & $0.988 \pm 0.006$ \\
\hline$\varepsilon_{o p p_{-} q}$ & $0.988 \pm 0.001$ \\
\hline$f_{b b}$ & $0.005 \pm 0.003$ \\
\hline$f_{\text {cosmic }}$ & $0.002 \pm 0.002$ \\
\hline$f_{\tau \tau}$ & $0.005 \pm 0.001$ \\
\hline$f_{W}$ & $0.002 \pm 0.001$ \\
\hline
\end{tabular}

Table 4.7: Efficiencies and backgrounds taken from the inclusive $Z \rightarrow$ $\mu^{+} \mu^{-}$cross section

The Diffractive cross section obtained after applying correction factors and efficiences is $4.09 \pm 0.64$ (stat) pb. Systematic errors are studied in the next section. 


\subsubsection{Systematic Errors}

Systematic errors are calculated by varying the parameters of the analysis and comparing the value obtained from each variation with the results obtained from the optimal parameter values.

\section{Cell Energy Thresholds}

Cell energy thresholds were applied to electromagnetic and fine hadronic calorimeter cells, the thresholds were fixed to $E M=0.5 \mathrm{GeV}$ and $F H=0.6 \mathrm{GeV}$. These thresholds were applied to all the calorimeter cells. We study the effect produced in the diffractive cross section when the thresholds are changed by $0.05 \mathrm{GeV}$ for calorimeter cells. Table 4.8 shows the diffractive cross section obtained by varying cell thresholds.

\begin{tabular}{|c||c|}
\hline thresholds & Diffractive Cross Section $(p b)$ \\
\hline$E M=0.45 \mathrm{GeV}, \mathrm{FH}=0.55 \mathrm{GeV}$ & $4.22 \pm 0.65$ \\
\hline$E M=0.55 \mathrm{GeV}, \mathrm{FH}=0.65 \mathrm{GeV}$ & $4.94 \pm 0.67$ \\
\hline
\end{tabular}

Table 4.8: Z Diffractive Cross Section by varying cell energy thresholds in $0.05 \mathrm{GeV}$

According to the Table 4.8, the maximum variation occurs when the thresholds have been increased to $E M=0.55 \mathrm{GeV}, F H=0.65 \mathrm{GeV}$, the difference respect to the cross section is $\Delta \sigma=0.85$ (about 20\%). When the thresholds have been decreased to $E M=0.45 \mathrm{GeV}, F H=0.55 \mathrm{GeV}, \Delta \sigma=0.13$ (about 3\%). We can see that the cross section is too sensible to this variation. This fact can be explained for one reason: The cell energy thresholds have been applied to whole calorimeter, so, when the thresholds are increased, the energy deposited in the forward cells (antiproton side) by soft particles (i.e pions) could be smaller than the cell energy threshold, therefore, the event can be tagged as diffractive event with gap.

\section{Background}

Background subtraction was applied to events that pass the diffractive cuts, the background was calculated from the PYTHIA sample that was normalized to the data sample after applying non diffractive cuts (See Table 4.5). For systematic errors studies from background we modify the normalization constant that takes into account trigger effects in $\pm 1 \sigma$. Table 4.9 shows the diffractive cross section results by modifying the normalization constants. 
The diffractive cross section is not affected significantly (about 2.4\%) for this parameter because the number of non diffractive events that pass the diffractive requirement is too low.

\section{Gap Size}

For gap requirement, we have defined a region between $-3.2<\eta<-4.45$ with $\sum E=0$ from calorimeter cells. The events that pass this requirement at generation level are taken into account for the montecarlo efficiency. However, the calorimeter cells have a size (See DØ detector chapter) and the $\eta$ value for the gap corresponds to mean value of the $\eta$ position of the cell. Aditionally, forward calorimeter cells beyond $|\eta|>3.2$ have different sizes. We study this systematic error by modifying the gap size at generation level. We count the number of events with the gap size modified, the number of events that pass the gap modified is used to recalculate the montecarlo efficiency, therefore the diffractive cross section is affected. The gap size is associated to the cell size in the forward calorimeter cells. Table 4.10 shows the gap region and the diffractive cross section obtained.

According to Table 4.10, when the gap size is increased the cross section decreased due to the sum of energy requirement because the number of events that pass this requirement is reduced. Opposite situation ocurrs when the gap size is reduced. This systematic error modifies the diffractive cross section about $4.8 \%$.

\section{Integrated Luminosity}

The Integrated Luminosity measured by $\mathrm{D} \varnothing$ experiment has an uncertainty of $6.5 \%[66]$.

\begin{tabular}{|c||c|}
\hline Quantity & Diffractive Cross Section $(p b)$ \\
\hline$-1 \sigma$ & $4.19 \pm 0.64$ \\
\hline$+1 \sigma$ & $3.99 \pm 0.64$ \\
\hline
\end{tabular}

Table 4.9: Z Diffractive Cross Section by varying normalization constants for background in $\pm 1 \sigma$

\begin{tabular}{|c||c|}
\hline Gap Region & Diffractive Cross Section $(p b)$ \\
\hline$-4.3<\eta<-3.15$ & $4.30 \pm 0.67$ \\
\hline$-4.87<\eta<-3.05$ & $3.91 \pm 0.61$ \\
\hline
\end{tabular}

Table 4.10: Z Diffractive Cross Section by varying gap size 


\subsubsection{Results for Diffractive Cross Section with Gap}

Taking into account the systematic errors explained in the previous section, the diffractive cross section multiplied by branching ratio for events with gap and fraction of the (anti)proton momentum loss between $0.02<\xi<0.1$ is:

$\sigma_{\text {Diff }}^{\text {gap }} \times B r\left(Z / \gamma^{*} \rightarrow \mu^{+} \mu^{-}\right)=4.09 \pm 0.64($ stat. $) \pm 0.88($ syst. $) \pm 0.27($ lumi. $) p b$

Adding in quadrature all uncertainties we obtain:

$$
\sigma_{\text {Diff }}^{\text {gap }} \times \operatorname{Br}\left(Z / \gamma^{*} \rightarrow \mu^{+} \mu^{-}\right)=4.09 \pm 1.12 p b
$$

\subsection{Fraction of $\mathrm{Z}$ bosons produced diffractively with gap requirement from $\mathrm{Z}$ inclusive production}

We measure the fraction of the $\mathrm{Z}$ boson with gap produced diffractively from the $\mathrm{Z}$ inclusive boson production in the muon channel decay. To do this, the number of diffractive $\mathrm{Z}$ candidates is divided by the number of $\mathrm{Z}$ bosons. Both values are corrected by efficiencies and correction factors. However, the diffractive $\mathrm{Z}$ candidates coming from $\mathrm{Z}$ inclusive production and, the correction factors and efficiencies shown in the Table 4.7 are the same for both values. So, they only differ in the montecarlo efficiency. The $\mathrm{Z}$ boson inclusive sample is dominated by inelastic events (non diffractive events), so this sample is simulated with PYTHIA. The montecarlo efficiency for PYTHIA combines the geometrical acceptance and tranverse momentum requirement for the muons from $\mathrm{Z}$ boson and dimuon mass greater to $40 \mathrm{GeV}$. The montecarlo efficiency calculated for PYTHIA is:

$$
\left(\varepsilon_{M C}\right)_{\text {Pythia }}=0.230 \pm 0.001
$$

This value agrees with calculation made by other analysis [65], [67].

The montecarlo efficiency for diffractive $\mathrm{Z}$ events was calculated for POMWIG in the previous section $(0.052 \pm 0.002)$. The equation used in order to obtain the fraction of the diffractive $\mathrm{Z}$ boson with gap from $\mathrm{Z}$ boson inclusive production is:

$$
R_{\text {Diff }}^{\text {gap }}=\frac{\text { Diff. } Z /\left(\varepsilon_{M C}\right)_{\text {pomwig }}}{A l l Z /\left(\varepsilon_{M C}\right)_{\text {pythia }}}
$$

According to the values obtained previously, this ratio is: 


$$
R_{\text {Diff }}^{\text {gap }}=1.92 \pm 0.30(\text { stat }) \%
$$

The systematic errors are the same as explained for the diffractive cross section. Table 4.11 shows the quantities and ratios obtained by modifying cell threshods, background and gap size.

\begin{tabular}{|r|l||l|l||r|l|}
\hline \multicolumn{2}{|c||}{ Cell Thresholds } & \multicolumn{2}{c||}{ Background } & \multicolumn{2}{c|}{ Gap Size } \\
\hline \hline Thresholds (GeV) & Ratio (\%) & Quantity & Ratio (\%) & Gap Region & Ratio (\%) \\
\hline$E M=0.45, F H=0.55$ & $1.98 \pm 0.31$ & $-1 \sigma$ & $1.97 \pm 0.30$ & $-4.3<\eta<-3.15$ & $2.02 \pm 0.31$ \\
\hline$E M=0.55, F H=0.65$ & $2.32 \pm 0.31$ & $+1 \sigma$ & $1.88 \pm 0.30$ & $-4.45<\eta<-3.05$ & $1.84 \pm 0.29$ \\
\hline
\end{tabular}

Table 4.11: Systematic Errors for fraction of Diffractive $\mathbf{Z}$ boson from $\mathbf{Z}$ inclusive production

The ratio of diffractive $\mathrm{Z}$ from $\mathrm{Z}$ inclusive production, taking into account the systematic errors is:

$$
\left.R_{\text {Diff }}^{\text {gap }}=1.92 \pm 0.30(\text { stat. }) \pm 0.41 \text { (syst. }\right) \pm 0.12(\text { lumi }) \%
$$

Combining all the uncertainties in quadrature, we obtain:

$$
R_{\text {Diff }}^{\text {gap }}=1.92 \pm 0.52 \%
$$

This number is in agreement with published results using Tevatron Run I data for $Z \rightarrow e^{+} e^{-} \quad[49]$.

Note also that our gap fraction measurement agrees with the ratio between the diffractive $\mathrm{Z}$ cross section measured by us and the $\mathrm{Z}$ boson inclusive cross section measured in the $\mathrm{D} \varnothing$ experiment [44].

$$
\frac{\sigma_{\text {Diffractive Z }}}{\sigma_{\mathrm{Z} \text { inclusive }}}=\frac{4.09 \pm 1.09}{327.8 \pm 9.1} \times 100 \%=1.25 \pm 0.34 \%
$$




\section{Appendix A}

\section{Muon Quality Definitions}

Reconstructed muon candidates are classified using two parameters: muon type and muon quality. The type of muon is given by the parameter nseg. A positive value of $n s e g$ indicates that the muon reconstructed in the muon system ("local muon") was matched to a track in the central tracking system. A negative value of nseg means that the local muon could not be matched to a central track. The absolute value $|n s e g|=1,2$ or 3 respectively indicates that the local muon is detected by only hits in the A-layer, B or C-layer (B and C outside the Toroid), or both $\mathrm{A}$ and $\mathrm{B}$ or C-layers hits.

The second parameter used to classify muons is the quality. The muon quality can be "Loose","Medium" or "Tight". The definitions for Tight, Medium and Loose are given below [48]:

- Tight Muons

Only $\mid$ nseg $\mid=3$ muons can be Tight. The requirements for Tight Muons are:

- at least two A layer wire hits

- at least one A layer scintillator hit

- at least three BC layer wire hits

- at least one BC scintillator hit

- a converged local fit $\left(\chi^{2}>0\right)$

- $|n s e g|=3$ Medium/Loose Muons

When an $\mid$ nseg $\mid=3$ muon candidate fails the Tight criteria it might still be Medium or Loose. An $\mid$ nseg $\mid=3$ muon is Medium if it has:

- at least two A layer wire hits 
- at least one A layer scintillator hit

- at least two BC layer wire hits

- at least one BC scintillator hit (except for central muons with less than four $\mathrm{BC}$ wire hits).

An $\mid n$ seg $\mid=3$ Loose muon is defined as a Medium muon but allowing one of the above tests to fail, with the A wire and scintillator requirement treated as one test and requiring always at least one scintillator.

\section{- $n s e g=+2$ Loose/Medium Muons}

Muons with $|n s e g|<3$ can only be Loose or Medium if they are matched to a central track. $n s e g=2$ muons are muons with a BC segment matched with a central track. Loose requires:

- at least one BC layer scintillator hit

- at least two BC layer wire hits

An $n s e g=2$ muon is defined as Medium if it fulfills the above requirements and if it is located in the bottom part of the detector (octant 5 and 6 with $\left.\eta_{\text {detector }}<1.6\right)$.

- $n s e g=+1$ Loose/Medium Muons

Muons with $n$ seg $=1$ are muons with an A segment matched with a central track. Annseg $=1$ muon is Loose if it has:

- at least one scintillator hit

- at least two A layer wire hits 


\section{Appendix B}

\section{Trigger Names}

The trigger names are composed of abbreviations of the different trigger components. Single Muon Triggers were used in this analysis in order to selected $Z / \gamma^{*} \rightarrow \mu^{+} \mu^{-}$candidates. The [Table B.1] shows the abbreviated names and what they represent.

\begin{tabular}{|c||l|}
\hline Component Name & Description \\
\hline \hline 2MU & two Level 1 tight scintillator muons \\
\hline MU & one Level 1 tight scintillator muon \\
\hline MUW & one Level 1 tight scintillator muon + loose wire muon \\
\hline A & Level 1 muons can be anywhere \\
\hline W & Level 1 muons have $\left|\eta_{A}\right|<1.5$ \\
\hline L2M0 & one Level 2 medium muon \\
\hline L2M(3,5) & one Level 2 medium muon, $p_{T}>(3,5) \mathrm{GeV}$ \\
\hline L2ETAPHI & two well separated Level 2 medium muons \\
\hline TRK(3,5,10) & one Level 3 central track, $p_{T}>(3,5,10) \mathrm{GeV}$ \\
\hline $\mathbf{2 T R K 3}$ & two Level 3 central tracks, $p_{T}>3 \mathrm{GeV}$ \\
\hline L3L(6,15) & one Level 3 muon, $p_{T}>(6,15) \mathrm{GeV}$ \\
\hline
\end{tabular}

Table B.1: Description of trigger components used for trigger names 


\section{Appendix C}

\section{Efficiencies and Corrections Factors for Inclusive $\mathrm{Z}$ boson Production}

Efficiencies and correction factors are taken from Z boson inclusive analysis [44]. We describe the procedure in order to obtain the corrections and efficiencies.

\section{C.1 Efficiency of isolation cuts $\varepsilon_{i s o l}$}

Figure Fig C.1 shows $M_{\mu \mu}$ for the events that are rejected exclusively by the isolation cuts (plain histogram) and the expected shape and size of $M_{\mu \mu}$ for $Z / \gamma^{*} \rightarrow$ $\mu^{+} \mu^{-}$events rejected by the isolation cuts (blue solid histogram). The shape of the blue histogram is taken from signal events in the category where at least one of isolation cuts fail. The size was found by fitting the histogram by eye to the region near $m_{Z}$ in the plain histogram. By integrating over the blue shaded histogram, the number of signal events rejected by the isolation cuts correspond to a fraction of 0.001 of the final sample. The efficiency for the isolation cuts is found to be $\varepsilon_{\text {iso }}=0.999 \pm 0.001$, where a $100 \%$ uncertainty has been assigned to the number of signal events rejected by these cuts.

\section{C.2 Efficiency of Opposite Sign Requirement and $b \bar{b}$ Background}

Figure Fig C.2 shows a distribution in $M_{\mu \mu}$ for the events exclusively rejected by the requirement that the two muons have opposite sign charge. This cut removes 


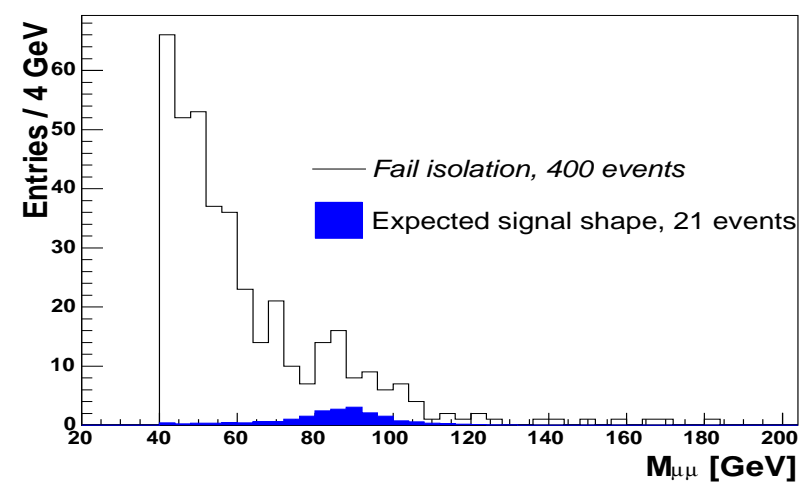

Figure C.1: Distribution of $M_{\mu \mu}$ for events exclusively rejected by the isolation cuts. Blue shaded histogram: Expected shape and size of $M_{\mu \mu}$ for signal events rejected by the isolation cuts

a fraction of 0.0047 of the potential candidate event sample. Some of these events are likely to originate from $b \bar{b}$ background where the muons are buried inside jets and so should appear non-isolated from other particles.

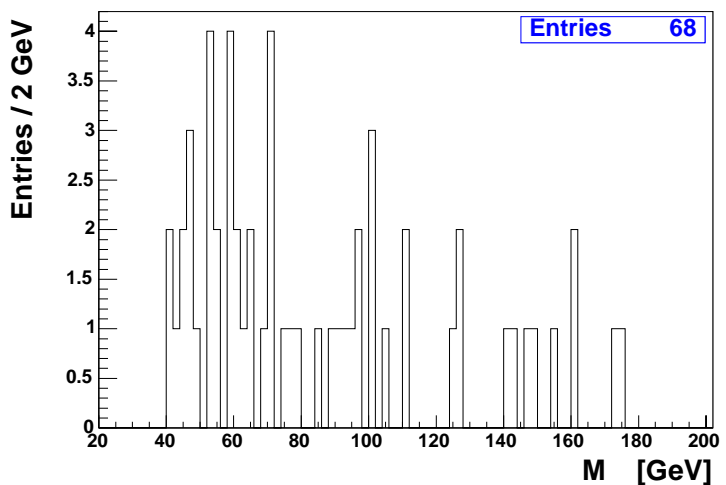

Figure C.2: Distribution of $M_{\mu \mu}$ for the events exclusively rejected by the requirement that the two muons be oppositely charged.

Although most of the muons from candidate $Z / \gamma^{*} \rightarrow \mu^{+} \mu^{-}$events will be isolated from other particles, the presence of jets may cause one or both of the muon to be non-isolated. In order to check the purity of the candidate sample, the distributions of $M_{\mu \mu}$ for events falling into the following three categories are 
compared:

- Both muons are isolated in both the central trackers and the calorimeter.

- At least one of the muons is isolated in the central tracker and non-isolated in the calorimeter.

- Any events not in categories (1) and (2).

Figure Fig C.3 shows the distribution of $M_{\mu \mu}$ for candidate events with both muons isolated.

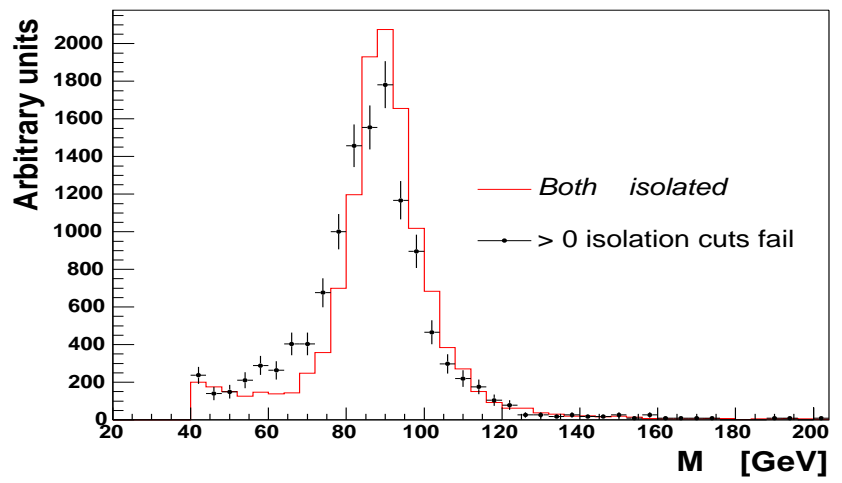

Figure C.3: Distribution of $M_{\mu \mu}$ for events with two isolated muons. The distributions are normalized to the same number of events in the region $M_{\mu \mu}>50 \mathrm{GeV}$

Also, $Z / \gamma^{*} \rightarrow \mu^{+} \mu^{-}$events that fail the opposite charge requirement can come from events in which the sign of the curvature for one of the two muons has been mis-measured. The fraction of events exclusively rejected by this cut is 0.0023. So, the efficiency for the opposite charge and the fraction for $b \bar{b}$ background are respectively:

$$
\varepsilon_{\text {opp } q}=0.998 \pm 0.001, \quad f_{b b}=0.005 \pm 0.003 .
$$




\section{C.3 Efficiency and Background for Cosmic Rays}

The difference in the times measured in the A-layer scintillator ${ }^{1}$ by the two muons, $\Delta t_{A}$, should be small for $Z / \gamma^{*} \rightarrow \mu^{+} \mu^{-}$events, since the muons should take approximately the same time to reach the A-layer. The time for a cosmic muon to transverse the detector will be $\sim 20 \mathrm{~ns}$. Cosmic events would be expected to have a small $\Delta \alpha_{\mu \mu}$ and a high $\left|\Delta t_{A}\right|$. It is clear that the signal events are well separated from the cosmic events and that the choice of 0.05 for a cut on $\Delta \alpha_{\mu \mu}$ is a good one. The number of events with a high $\left|\Delta t_{A}\right|$ that appear to be in the tail of the cosmic $\Delta \alpha_{\mu \mu}$ distribution is small which suggest that the cosmic background is small [Fig C.4].

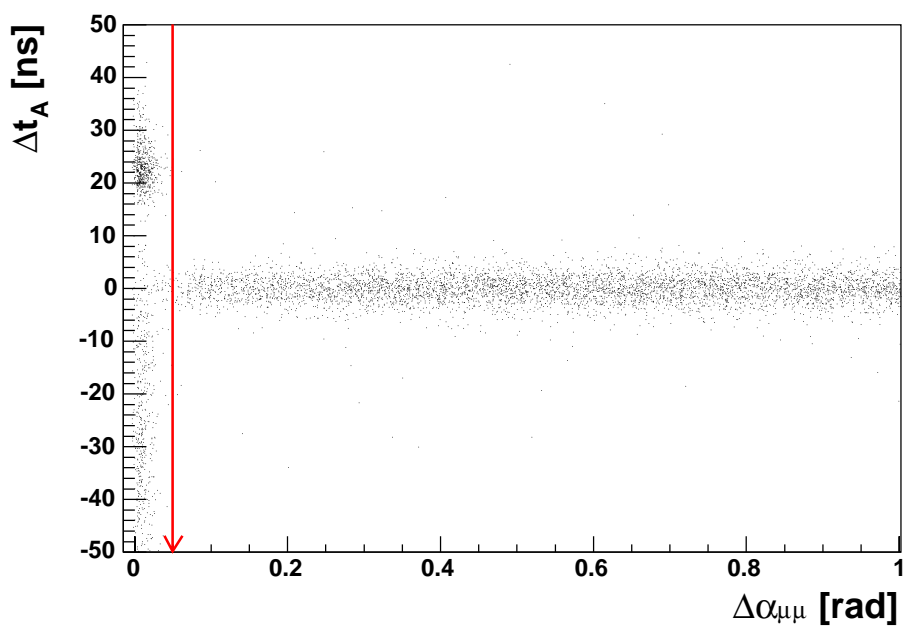

Figure C.4: $\Delta t_{A}$ versus $\Delta \alpha_{\mu \mu}$ for events that have passed all cuts but that on $\Delta \alpha_{\mu \mu}$. The red arrow indicates the position of the cut.

In order to cross check this, a plot of the speed of the muon is made as if it were a cosmic transversing the detector. The distance travelled is the distance between muon 1 and muon 2, measured in the muon detectors and the time is the difference between the scintillator times of muon 1 and muon 2. For a cosmic this would be expected to be near to the speed of light and for a $Z / \gamma^{*} \rightarrow \mu^{+} \mu^{-}$event it would be infinitely large. Figure [Fig C.5] shows the speed for candidate events.

\footnotetext{
${ }^{1}$ If either of the muons has not hits in the A-layer scintillators, then the time from the BC-layer scintillators is used. The time measured by the A and BC- layer scintillators is corrected for the expected time of flight of the muons to the respective layers so that it should be close to zero.
} 


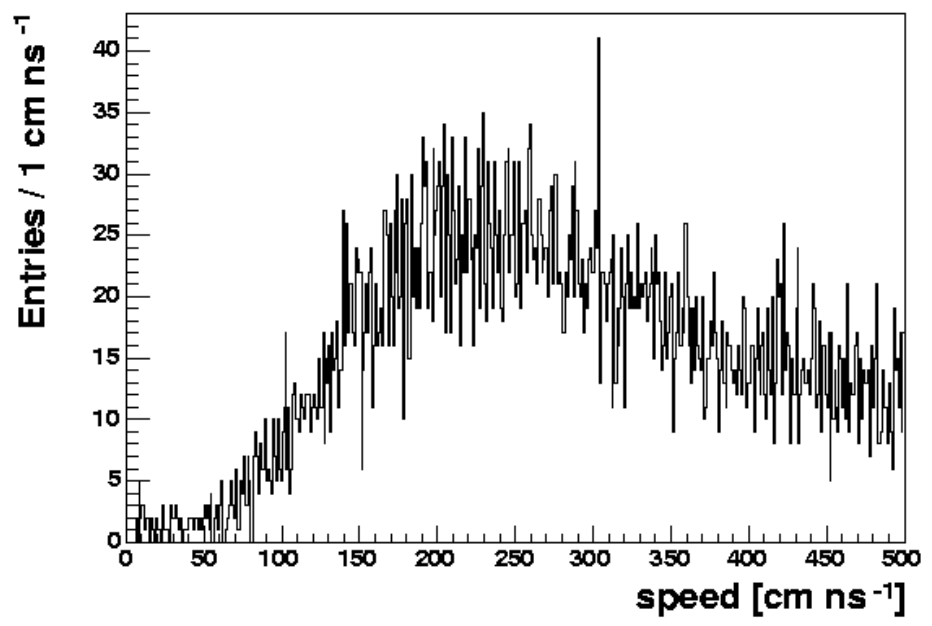

Figure C.5: Speed of muon pair in candidate events as if they were one muon transversing the detector

If half of the events with a speed between 0 and $50 \mathrm{~cm} \mathrm{~ns}^{-1}$ are assumed to be from cosmic events and half of them from $Z / \gamma^{*} \rightarrow \mu^{+} \mu^{-}$events in the tail of the distribution, the fractional background from cosmic events is estimated to be

$$
f_{\text {cos }}=0.002 \pm 0.002 \text {, }
$$

where a relative $100 \%$ uncertainty to the number of background events has been assigned.

Figure [Fig C.6] shows the distribution of $M_{\mu \mu}$ for events rejected by the $\Delta \alpha_{\mu \mu}$ and dca cuts. Inspection of this plots shows that most of the rejected events are background.

The efficiency of the $\Delta \alpha_{\mu \mu}$ cut is measured using PMCS to be 0.004 .

There are signal events that fail the dca cut due to mis-reconstructed mass as a poor resolution in $p_{T}$ would be expected for tracks with a large $d c a$. In order to estimate the amount of signal events rejected by this cut, a plot of the $d c a$ of one of the muon tracks is plotted against that of the other muon track before the dca cuts have been applied, as shown in figure [Fig C.7]

The fraction of the signal events that fail dca cuts exclusively is 0.008. So, the total fraction of events rejected by the cosmic cuts $\left(\Delta \alpha_{\mu \mu}\right.$ and dca) is estimated to be $0.004+0.008=0.012$. An efficiency for the cosmic cuts of $\varepsilon_{\text {cosmic }}=0.988 \pm 0.006$ is obtained. A relative $50 \%$ uncertainty has been assigned to the estimation of the number of events rejected by these cuts. 


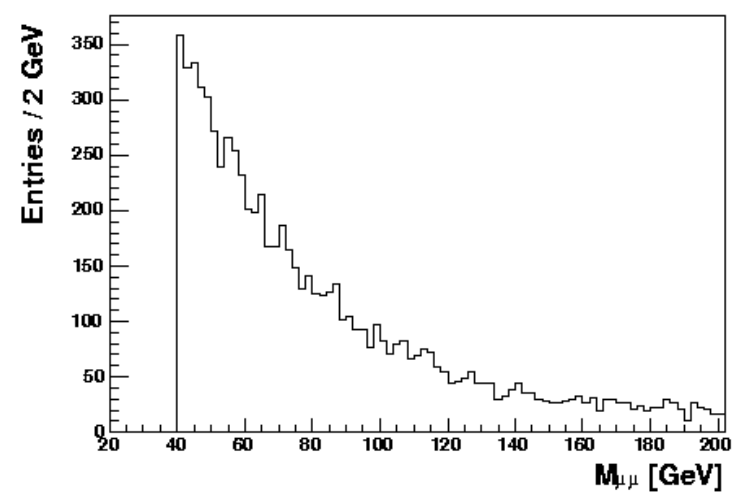

Figure C.6: Distribution of $M_{\mu \mu}$ for the events rejected by the cosmic muons (dca or $\Delta \alpha_{\mu \mu}$ )

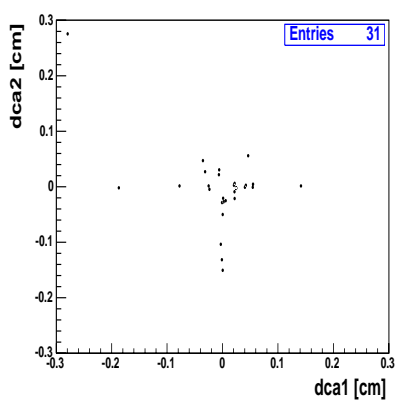

(a)

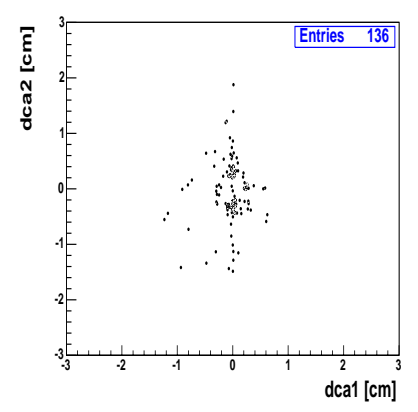

(b)

Figure C.7: Distribution of dca for one muon track versus dca for the other muon track for events that fail the dca cut but have passed all other cuts, with the additional requirement that all four isolation cuts pass. (a) For events where both tracks have SMT hits. (b) For events where at least one track has no SMT hits.

\section{C.4 $Z / \gamma^{*} \rightarrow \tau^{+} \tau^{-}$Background}

$Z / \gamma^{*} \rightarrow \tau^{+} \tau^{-}$events in which both taus decay to a muon could mimic a $Z / \gamma^{*} \rightarrow$ $\mu^{+} \mu^{-}$events. The $p_{T}$ spectrum of the muons will be softer than that in $Z / \gamma^{*} \rightarrow$ $\mu^{+} \mu^{-}$events but some events could pass the selection criteria. The number of 
$Z / \gamma^{*} \rightarrow \tau^{+} \tau^{-}$for every $Z / \gamma^{*} \rightarrow \mu^{+} \mu^{-}$event is $0.0051 \pm 0.0003$ by comparing the relative numbers of $Z / \gamma^{*} \rightarrow \mu^{+} \mu^{-}$and $Z / \gamma^{*} \rightarrow \tau^{+} \tau^{-}$DMCS events passing the selection criteria and $0.00408 \pm 0.00008$ by comparing the relative numbers of $Z / \gamma^{*} \rightarrow \mu^{+} \mu^{-}$and $Z / \gamma^{*} \rightarrow \tau^{+} \tau^{-}$PMCS events passing the sekection criteria. The difference between the two values is taken to be a systematic uncertainty so that a value $f_{\tau \tau}=0.005 \pm 0.001$ is obtained.

\section{C.5 Background from $\mathrm{W}+$ Jets and Di-boson Events}

$W \rightarrow \mu \nu$ events with an additional high $p_{T}$ muon from a jet could pass the event selection criteria. If the fraction of $W \rightarrow \mu \nu$ events with a high $p_{T}$ muon from a jet is assumed to be roughly the same as the fraction of $Z / \gamma^{*} \rightarrow \mu^{+} \mu^{-}$events with a high $p_{T}$ muon from a jet, this background can be studied using candidate events. The total fractional background from $W+$ jets and di-boson events is found to be: $f_{W}=0.002 \pm 0.001$. 


\section{Appendix D}

\section{The T42 and Hot Cell Killer Algorithms}

The T42 algorithm has been introduced [27] to enhance the treatment of the calorimeter noise. This leads to an improvement in the reconstruction of different objects (electrons, photons,jets, $\mathbb{E}_{T}$ ), whose identification and energy measurement relies mainly on the calorimeter.

Calorimeter noise, generally defined as energy deposition not related to the hard interaction, can be schematically classified as "hot", "warm" or "normal".

- Hot noise are related to detector problems (hardware failure, abnormal electronic noise), or to physics processes like backscattering of particles interacting in the beampipe outside of the vertex interaction region into the calorimeter. Their energy is typically large, $>1 \mathrm{GeV}$.

- Warm Noise Warm cells are due to pedestal subtraction problems or hardware deficiencies. The cell energy levels are typically lower, on the order of hundred of $\mathrm{MeV}$, however, they might appear in great numbers in a definite region of the detector, creating so-called warm zones.

- Normal Noise Normal noise cells are due to Gaussian electronic noise surviving the zero suppression. They are at lower energies, typically below $4-5 \sigma$, where $\sigma$ is the RMS of the pedestal. Typically, between 1000 and 3000 such cells appear per event.

The T42 algorithm is implemented in the d0reco code [30] and is applied before reconstructing the calorimeter objects. It aims to reject "normal" noise cells. For the T42 algorithm, an isolated cell is considered a noise cell and thus discarded 
if it is not "signal-like". A cell is considered "signal-like" if its energy is positive (negative energy cells can originate from electronics noise and from pile-up which is baseline subtracted) and above a high threshold of $+4 \sigma$, or if its energy is above $+2.5 \sigma$ and the energy of a neighboring cell is above $+4 \sigma$. The acronym T42 stands for "threshold $4 \sigma-2 \sigma$, however, the current implementation corresponds to "threshold $4 \sigma-2.5 \sigma$ ".

The first electromagnetic layer (layer 1), and the layers 8, 9 and 10 of the intercryostat region are not considered by the algorithm; so all cells in those layers with positive energy are kept in the event, and are not used as neighbors. A detailed description of the current implementation of the T42 algorithm can be found in [28].

The ratio of rejected cells by T42 over the number of cells in the event ranges from $30 \%$ to $60 \%$. In the main part of the calorimeter $(|\eta|<3.2)$, the fraction of cells rejected by T42 corresponds to the number of cells expected from noise between 2.5 and $4 \sigma$, assuming a Gaussian distribution [29]. This is a good indication that T42 is indeed reducing mainly noise cells. In the forward region, more cells than expected are rejected, since cells from pile-up effects accumulate close to the beam-pipe [29].

Hot noise also known as hot cells are excluded by using the Hot Cell Killer algorithm called NADA [31]. NADA uses a stronger criteria by considering a larger number of neighbors in the $\eta-\phi$ plane and different energy thresholds. In NADA the neighbor cells are defined within a cube surrounding a candidate cell (i.e. $E_{\text {cand }}>E_{\text {threshold }}$ ) with a $0.3 \times 0.3$ size in the $\eta \times \phi$ plane (see Figure [Fig D.1]).

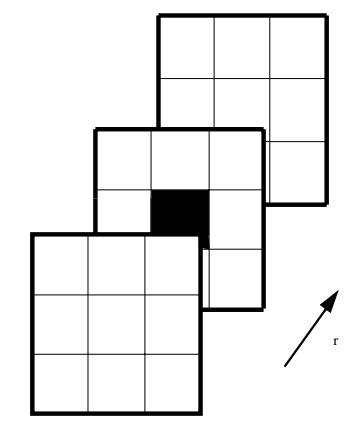

Figure D.1: Cube definition surrounding a candidate hot cell in $\eta \times \phi$ plane. 
The energy of the cube,

$$
E_{\text {cube }}=\sum_{i} E_{\text {cell }}^{i}>E_{\text {cell }}^{\text {cut }}
$$

excludes in the sum the central candidate and the cells which have an energy $E_{\text {cell }}^{i}<E_{\text {cell }}^{\text {cut }}$ cell in order to avoid the contribution of cells with low energy due for example to electronics noise. The candidate cell is then identified as a hot cell if the energy in the cube is lower than the parameter $E_{\text {cube }}^{c u t}$ :

$$
E_{\text {cube }}<E_{\text {cube }}^{\text {cut }} .
$$

The two parameters $E_{\text {cell }}^{\text {cut }}$ and $E_{\text {cube }}^{\text {cut }}$ cube can be optimized according to the chosen value of $E_{\text {threshold }}$ (used to determine the initial list of hot cell candidates) and/or to the position of a candidate cell in the calorimeter central or forward region in $\eta$ ).

Due to the geometry of the calorimeter some layers need a specific treatement The third electromagetic layer (EM3) has a twice better segmentation in $\eta \times \phi$ compared to the other layers $(0.05 \times 0.05$ instead of $0.1 \times 0.1)$. In the NADA algorithm cells of $0.05 \times 0.05$ size are merged by groups of four to form $0.1 \times$ 0.1 "cells". The first fine FH and the first coarse $\mathrm{CH}$ layers have higher energy depositions than their neighbors due to their higher nuclear interaction length and therefore need specic values of the parameters. ICD and Massless Gap layers belong to the transition region between the central and endcap cryostats where a relative large amount of uninstrumented material can be found due to cryostat walls and the calorimeter modules support structures. These layers will also need specific values of the parameters.

The NADA algorithm has been implemented and is available in the RunII reconstruction (d0reco) [30]. 


\section{Bibliography}

[1] S.L.Glashow Nucl.Phys. B 22,579 (1961)

[2] S. Weinberg Phys. Rev. Lett 1264 (1967)

[3] A. Salam, in Elementary Particle Theory edited by N. Svartholm Almqvist and Wiksells Stockholm p.367

[4] S. Drell and T. Yan. Ann Phys. 66. (1971)

[5] S. Eidelman et al. [Particle Data Group Collaboration], Phys. Lett. B 592 (2004) 1.

[6] Thompson, J.: Introduction to Colliding Beams at Fermilab. FERMILAB-TM-1909, 1994

[7] Edmunds, D.: RunII a Tevatron Beam Structure. http://www.pa.msu.edu/hep/d0/ftp/l1/framework/drawings/run iia beam structure.ps

[8] DØ Collaboration: The Upgraded DØ Detector. hepph/0507191, 2005.

[9] Particle Data Group Collaboration: Phys. Lett. 1B, 592, 2004

[10] C-C.Miao: FERMILAB-CONF-98-395-E, 1998.

[11] T. Edwards et al.: FERMILAB-TM-2278-EB, 2004.

[12] E. Kajfasz: DØ Silicon Microstrip Tracker for RunIIa. hepex/0112014, 2001.

[13] DØ Collaboration: The DØ Upgrade: Central Tracker Technical Design Report. 1999. http://d0server1.fnal.gov/users/stefan/www/CFT_TDR/CFT_ TDR.ps. 
[14] J. Brzenziak et al: FERMILAB-TM-1886, 1994.

[15] D. Whiteson and M. Kado: Muon Isolation Studies, D-Note 4070, December 2002.

[16] Adams, M. et al.: Design Report of the Central Preshower Detector for the Do Upgrade.1996. http://d0server1.fnal.gov/users/qianj/CPS/doc/dn3104.pdf.

[17] L. Groers: DØ Calorimeter Upgrades for Tevatron Run II DNote 4240 Proceedings for the IXth International Conference on Calorimetry in Particle Physics, Annecy, France, Oct 9-14, 2000.

[18] DØ Collaboration: Nucl. Instrum. Meth. A, 324/53, 1993.

[19] http://www-d0.fnal.gov/computing/algorithms/calgo/calgo_docs.html

[20] J. Brzenziak et al: FERMILAB-TM-1886, 1994.

[21] T. Diehl et al.: Technical Design of the Central Muon System DØ-Note 3365, March 1998.

[22] T. Diehl et al.: Technical Design for the D Forward Muon Tracking Detector Based on Mini-Drift Tubes DØ-Note 3366, December 1997.

[23] T. Diehl et al.: Technical Design Report for the D forward trigger scintillator counters DØ-Note 3237, November 1987.

[24] J. Buttler: Local Muon Momentum Resolution D-Note 4002, July 2002.

[25] 100. A. Brandt et al: Fermilab-Pub-97-377, 1997.

[26] U. Amaldi et al.: Phys. Lett. B 43/231, 1973.

[27] U. Bassler and G. Bernardi: Towards a Coherent Treatment of Calorimetric Energies,DØ-Note 4124, March 2003.

[28] J-R. Vlimant, U. Bassler, G. Bernardi and S. Trincaz-Duvoid: Technical description of the T42 algorithm for the calorimeter noise suppression, DØ-Note 4146, May 2003.

[29] G. Bernardi, E. Busato and J-R. Vlimant: Improvements from the T42 Algorithm on Calorimeter Objects Reconstruction, DØNote 4335, January 2004. 
[30] http://www-d0.fnal.gov/d0dist/dist/packages/d0reco/

[31] Bob Olivier, Ursula Bassler, Gregorio Bernardi. NADA. A New Event by Event Hot Cell Killer. DØ Note 3687. 2000

[32] A. Schwartzman and M. Narain: Secondary Vertex Reconstruction using the Kalman Filter DØ-Note 3908, September 2001.

[33] A. Garcia-Bellido et al: Primary Vertex certication in p14 D-Note 4320, January 2004.

[34] DØ Collaboration: Common Sample Group (CSG). http://wwwd0.fnal.gov/Run2Physics/cs/index.html.

[35] DØ Collaboration: Physics Letters B 626 55, 2005.

[36] W. M. Yao et al. The Review of Particle Physics. Journal of Physics, G 33, 1, 2006.

[37] G. Steinbrück. Measurement of the Angular Distribution of Electrons from W Boson Decays at D $\varnothing$. PhD thesis, University of Oklahoma, 1999.

[38] V. Buescher and J. Zhu. EM Certification Tools. DØ Note 4171, 2003.

[39] The EM ID Working Group. D EM ID Certification. http://wwwd0.fnal.gov/phys_id/emid/d0_private/certification/main_v5_0.html.

[40] E. Busato and B. Andrieu. Jet Algorithms in the DØ Run II Software: Description and User's Guide. DØ Note 4457, 2004.

[41] G. Blazey et al. Run II Jet Physics. Proc. of the QCD and Weak Boson Physics in Run II Workshop, 1999.

[42] B. Olivier et al. The Missing Transverse Energy Resolution of an Event. DØ Note 3629, 1999.

[43] G. Ingelman and P. E. Schlein, Phys. Lett. B 152 (1985) 256

[44] E.M Nurse - FERMILAB THESIS 2005-05

[45] Linda Coney, Difractive $\mathbf{W}$ and $\mathbf{Z}$ boson production in $p \bar{p}$ collisions - FERMILAB THESIS 2000-04

[46] http://www-d0.fnal.gov/trigger_meister/ 
[47] J. C. Collins, Phys. Rev. D 57 (1998) 3051 [Erratum-ibid. D 61 (2000) 019902] [arXiv:hep-ph/9709499]

[48] Muon Identification Certification for p17 data, DØ Note 5157

[49] V. M. Abazov et al. [DØ Collaboration], Phys. Lett. B 574, 169 (2003) [arXiv:hep-ex/0308032]

[50] Tamsin Edwards, Diffractively produced $\mathrm{Z}$ bosons in the muon decay channel and the measurement of the efficiency of the DØ Run II Luminosity Monitor - FERMILAB THESIS 2006

[51] John Anderson, et al. Upgrade of the DØ Luminosity Monitor Readout System - DØ Note 5297

[52] B. E. Cox and J. R. Forshaw, Comput.Phys.Commun.144:104110,2002

[53] http://hepwww.rl.ac.uk/theory/seymour/herwig/

[54] C. Adlo et al. [H1 Collaboration], Z. Phys. C 76 (1997) 613 [arXiv:hepex/9708016].

[55] http://www-d0.fnal.gov/computing/MonteCarlo/generator_tools/lhapdf.html

[56] http://www.phys.psu.edu/ cteq/

[57] http://www-d0.fnal.gov/computing/MonteCarlo/MonteCarlo.html

[58] http://wwwasd.web.cern.ch/wwwasd/geant/

[59] ThumbNail: a compact data format - DØ Note 3979

[60] Recommendations Regarding Common Analysis Format Content - DØ Note 4647

[61] http://www.thep.lu.se/ torbjorn/Pythia.html

[62] Abe et al. [CDF collaboration]. Phys.Rev. D 50 (1994) 5550

[63] C. Avila et al. [E811 Collaboration]. Phys.Lett. B 445 (1999) 419

[64] S.Klimenko, J. Konigsberg and T.M. Liss, Averaging of the inelastic cross sections measured by the CDF and the E811 experiments, FERMILAB-FN-0741 (2003) 
[65] Measurement of the Cross section for Inclusive $\mathbf{Z}$ Production in Di-muon Final States at $\sqrt{s}=1.96 \mathrm{TeV}$, Conference Note 4573. 2004

[66] http://www-d0.fnal.gov/runcoor/RUN/run2_lumi.html

[67] A Measurement of the $Z^{0}$ Boson Production Cross Section Times Muon Branching Ratio in $p \bar{p}$ Collisions at $1.96 \mathrm{TeV}$. FERMILAB THESIS 2003 